\title{
Modelos arch heterogêneos e aplicações à análise de dados de alta freqüência
}

\author{
Juan Carlos Ruilova Terán
}

TESE APRESENTADA AO

INSTITUTO DE MATEMÁTICA E ESTATÍSTICA DA

UNIVERSIDADE DE SÃO PAULO

PARA OBTENÇÃO DO GRAU DE

DOUTOR EM CIÊNCIAS

\author{
Área de concentração: Estatística \\ Orientador: Prof. Dr. Pedro A. Morettin
}

Durante a elaboração deste trabalho, o autor recebeu apoio financeiro da $\mathrm{CNPq}$

São Paulo, fevereiro 2007. 


\section{Modelos arch heterogêneos e aplicações à análise de dados de alta freqüência}

Este exemplar corresponde à redação final da tese devidamente corrigida e defendida por Juan Carlos Ruilova T. e aprovada pela Comissão Julgadora.

São Paulo, 26 de abril de 2007.

Banca Examinadora:

Prof. Dr. Pedro Alberto Morettin - IME-USP

Prof. Dr. Pedro Luiz Valls Pereira - IBMEC-SP

Prof. Dr. Luiz Koodi Hotta - IMECC-UNICAMP

Prof. Dra. Beatriz Vaz de Melo Mendes - UFRJ

Prof. Dr. Cristiano Augusto Coelho Fernandes - PUC-RJ 
Para meus pais: Carlos Ruilova e Rita Terán de Ruilova 


\section{Agradecimentos}

Desejo expressar meus mais sinceros agradecimentos a meus pais, que são eles que desde pequeno me incentivaram no caminho do estudo. Esta tese está dedicada a eles por todo esse esforço, amor e dedicação durante vários anos de minha vida, e essa luta incansável que sustiveram para nos dar o melhor para mim e meus irmãos.

Agradeço também a minha querida esposa Fabiana e meu filho Fausto (Fazinho), por todo seu amor, ajuda e paciência durante a elaboração desta tese. Sem o carinho, compreensão e colaboração deles teria sido impossível terminá-la.

Mudar de país é certamente um desafio e por isso quero agradecer a meus irmãos Fausto, Gabriel, Sebastián e María Fernanda e a meus amigos Freddy, Robert e William que me encorajaram para me embarcar nesta aventura.

Os equatorianos somos muito nostálgicos e sendo eu também assim morar num país distante pode resultar muito difícil, mesmo sendo o Brasil muito parecido ao Equador, por isso quero muito agradecer aos brasileiros pela sua hospitalidade e simpatia, especialmente aos grandes amigos que eu fiz aqui, Felipe, Thales, Flavio e Adriano pelos momentos de descontração e seu apoio nos momentos difíceis.

Quero também agradecer a meu orientador, o Professor Pedro A. Morettin, pelas muitas sugestões, confiança e constante ajuda durante a elaboração deste trabalho. Ele tem sido mais de que um orientador, pois tem me ajudado não somente no âmbito acadêmico, mas também no meu crescimento como pessoa.

Por último, quero também agradecer à agencia financiadora $\mathrm{CNPq}$ do Brasil que forneceu os recursos necessários para minha permanência no Brasil. 


\section{Resumo}

Neste trabalho estudamos diferentes variantes dos modelos GARCH quando consideramos a chegada da informação heterogênea sob a forma de dados de alta freqüência. Este tipo de modelos, conhecidos como $\operatorname{HARCH}(n)$, foram introduzidos por Muller et al. (1997).

Para entender a necessidade de incorporar esta característica da heterogeneidade da informação, estudamos o problema da agregação temporal para processos GARCH e a modelagem destes em dados de alta freqüência e veremos quais são as desvantagens destes modelos e o porquê da necessidade de corrigi-lo.

Propusemos um novo modelo que leva em conta a heterogeneidade da informação do mercado financeiro e a memória longa da volatilidade, generalizando assim o modelo proposto por Müller et al.(1997), e estudamos algumas das propriedades teóricas do modelo proposto.

Utilizamos estimação via máxima verossimilhança e amostrador de Griddy-Gibbs, e para avaliar o desempenho destes métodos realizamos diversas simulações.

Também fizemos aplicações a duas séries de alta frequiência, a taxa de câmbio EuroDólar e o índice Ibovespa.

Uma modificação ao algoritmo de Griddy-Gibbs foi proposta, para ter uma janela móvel de pontos, para a estimação das distribuições condicionais, a cada iteração. Este procedimento foi validado pela proximidade das estimações com a técnica de máxima verossimilhança.

Disponibilizaremos algumas bibliotecas para o pacote S-Plus em que as análises descritas neste trabalho poderão ser reproduzidas. Informações relativas a tais bibliotecas estarão disponíveis na página Web'http://Www.ime.usp.br/ ruilova 


\section{Abstract}

In this work we study different variants of GARCH models to analyze the arrival of heterogeneous information in high frequency data. These models, known as HARCH $\left(*^{*} *\right)$ models, were introduced by Müller et al.(1997).

To understand the necessity to incorporate this characteristic, heterogeneous information, we study temporal aggregation on GARCH processes for high frequency data, and show some problems in the application of these models and the reason why it is necessary to develop new models.

We propose a new model, that incorporates the heterogeneous information present in the financial market and the long memory of the volatility, generalizing the model considered by Müller et al.(1997).

We propose to estimate the model via maximum likelihood and Griddy-Gibbs sampler. To assess the performance of the suggested estimation procedures we perform some simulations and apply the methodology to two time series, namely the foreign exchange rate Euro-Dollar and the series of the Ibovespa index.

A modification of the algorithm of Griddy-Gibbs sampler was proposed to have a grid of points in a mobile window, to estimate the condicional distributions, in each iteration. This was validated by the similar results between maximum likelihood and Griddy-Gibbs sampler estimates obtained.

We implemented the methods described in this work creating some libraries for the SPlus package. Information concerning these libraries is available in the Web page http://www.ime.usp.br/ ruilova. 


\section{Sumário}

MOTIVAÇÃO E CONTRIBUIÇÃO ---

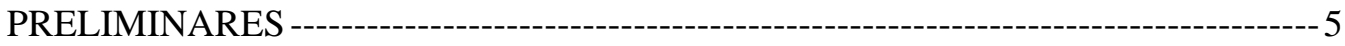

1.1. DADOS DE ALTA FREQÜÊNCIA. ------------ 5

1.2. VOLATILIDADE E TENDÊNCIAS. --------------- 6

1.2.1. MODELOS TIPO ARCH.----------- 7

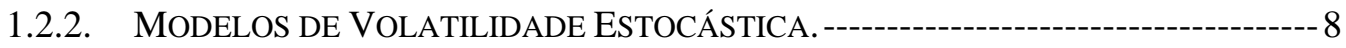

1.2.3. MODELOS BASEADOS NA VOLATILIDADE REALIZADA.-------------------------- 8

1.2.4. MODELOS DE VOLATILIDADE HISTÓRICA. -------------------------------- 8

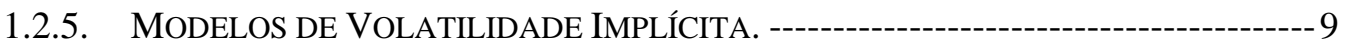

1.2.6. TREND FOLLOWING. ------ 9

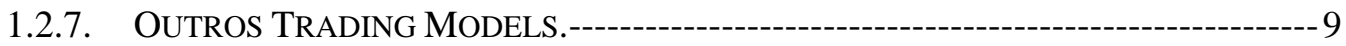

1.3. Alguns RESUltadOS SOBRE CADEIAS DE MARKOV. ------------------------ 10

MODELAGEM DE DADOS DE ALTA FREQÜÊNCIA ------------------------------- 21

2.1. INTRODUÇÃO. --- 21

2.2. MODELOS GARCH.

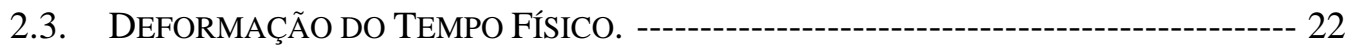

2.4. AGREGAÇÃO TEMPORAL. --------- 24

2.5. APLICAÇÕES DA AGREGAÇÃO TEMPORAL. --- 33

2.5.1. PRINCIPAIS FATOS ESTILIZADOS DAS SÉRIES FINANCEIRAS. ----------------- 33

2.5.2. ESTIMAÇÃO DE MODELOS GARCH. ------------ 44

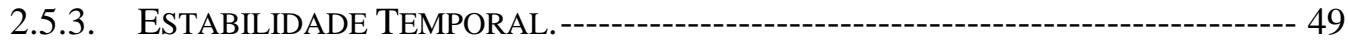

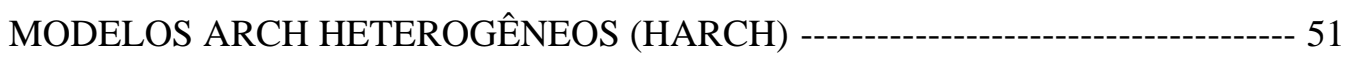

3.1. INTRODUÇÃO. --- 51

3.2. O MODELO HARCH.---------- 52

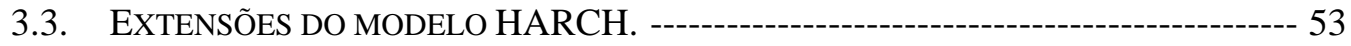


3.4. ESTACIONARIEDADE. CONDIÇÕES NECESSÁRIAS. ------------------------------ 55

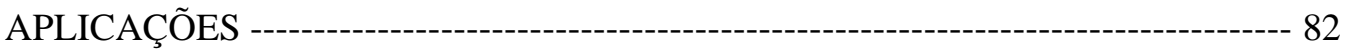

4.1. INTRODUÇÃO. -- 82

4.2. AMOSTRADOR GRIDDY-GIBBS. -------------- 83

4.3. SIMULAÇÃO. ------------------------------------- 83

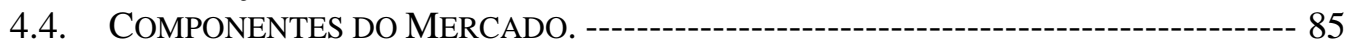

4.5. ESTIMAÇÕES DE MÁXIMA VEROSSIMILHANÇA. --------------------------------- 86

4.6. ESTIMAÇÕES USANDO O AMOSTRADOR GRIDDY-GIBBS. ---------------------- 88

4.7. ESTIMAÇÕES DE MÁXIMA VEROSSIMILHANÇA PARA O ÍNDICE IBOVESPA E A

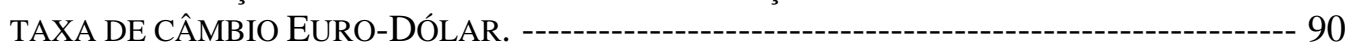

4.8. ESTIMAÇÕES POR MEIO DO AMOSTRADOR GRIDDY-GIBBS PARA O ÍNDICE

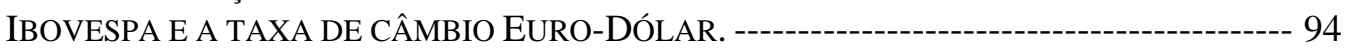

4.9. PREVISÕES PARA O ÍNDICE IBOVESPA E A TAXA DE CÂMBIO EURO-DÓLAR.---- 96

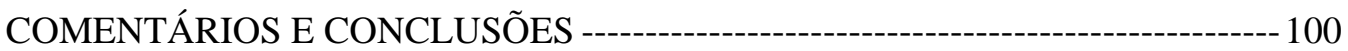

REFERÊNCIAS BIBLIOGRÁFICAS ----------------------------------------------------- 103 


\section{Prefácio}

\section{Motivação e contribuição}

Os dados de alta frequiência, aqueles medidos em intervalos pequenos de tempo ${ }^{1}$, têm tido muita importância durante os últimos anos, devido principalmente ao incremento nos avanços computacionais.

Em 1965, Gordon Moore, um dos fundadores da Intel, disse que "a complexidade dos microprocessadores, medida em número de transistores, dobra a cada 18 meses". Essa previsão logo foi batizada como a Lei de Moore, e indica que a velocidade de processamento cresce de maneira exponencial. Outras observações semelhantes indicam que a capacidade de armazenamento em disco tem dobrado a cada 9 meses, com custos sempre decrescentes, e que a cada 3 anos o tráfego e a complexidade das redes aumenta de forma a consumir o dobro da capacidade anterior.

Ao ter então, cada vez mais capacidade de armazenamento e processamento a menor custo, os dados de alta freqüência começaram a ser armazenados e disponibilizados de maneira natural. Estes dados trazem consigo mais informação, o que melhoraria as previsões.

Os modelos de séries de tempo tradicionais, quando usados em dados de alta freqüência, muitas vezes não têm se comportado de maneira adequada, obrigando a realizar adequações que tornariam estes modelos apropriados à informação de alta freqüência.

O modelo mais comumente usado para modelar a volatilidade em séries financeiras é o $\operatorname{GARCH}(1,1)$, que é dado pela seguinte equação:

\footnotetext{
${ }^{1}$ No ambiente financeiro, são geralmente consideradas como séries de alta freqüência aquelas séries medidas em freqüência diária ou intradiária.
} 


$$
\begin{aligned}
& r_{t}=\sigma_{t} \varepsilon_{t}, \quad \varepsilon_{t} \sim \operatorname{iid}(0,1) \\
& \sigma_{t}^{2}=\alpha_{0}+\alpha_{1} r_{t-1}^{2}+\beta_{1} \sigma_{t-1}^{2}
\end{aligned}
$$

$\operatorname{com} \alpha_{0}>0, \alpha_{1} \geq 0, \beta_{1} \geq 0, \alpha_{1}+\beta_{1}<1$.

No entanto, ele apresenta certos problemas quando é usado para modelar, por exemplo, séries financeiras em altas freqüências. Provavelmente pela heterogeneidade da chegada da informação nos mercados financeiros.

$\mathrm{O}$ modelo $\operatorname{HARCH}(n)$ foi criado para tentar solucionar este problema e incorporar a característica da heterogeneidade na informação das séries financeiras. O modelo $\operatorname{HARCH}(n)$, devido a Müller et al. (1997), é dado por:

$$
\begin{aligned}
& r_{t}=\sigma_{t} \varepsilon_{t}, \\
& \sigma_{t}^{2}=c_{0}+\sum_{j=1}^{n} c_{j}\left(\sum_{i=1}^{j} r_{t-i}\right)^{2},
\end{aligned}
$$

onde $c_{0}>0, c_{n}>0, c_{j} \geq 0 \forall j=1, \ldots, n-1$ e os $\varepsilon_{t}$ são variáveis aleatórias i.i.d. com média zero e variância um.

No entanto, este modelo teve pouca acolhida na literatura estatística. Isto foi devido principalmente ao grande custo computacional da sua modelagem, tanto na implementação e complexidade do algoritmo, quanto na velocidade de processamento, e ao pequeno ganho em relação a modelos como o GARCH.

Isto se deve, entre outras coisas, à característica de memória longa que apresenta a volatilidade, o que implica numa grande quantidade de parâmetros a estimar.

Nossa primeira contribuição é propor um novo modelo, que tome em conta a heterogeneidade da informação do mercado financeiro e a memória longa da volatilidade, além de generalizar o modelo proposto por Müller et al. (1997).

Propomos, então, o modelo $\operatorname{GHARCH}(m, p)$ parcimonioso, com agregações de tamanhos diferentes $a_{1}, \ldots, a_{m}$, onde $m$ é o número de componentes do mercado, dado por:

$$
\begin{aligned}
r_{t} & =\sigma_{t} \varepsilon_{t} \\
\sigma_{t}^{2} & =C_{0}+C_{1}\left(r_{t-1}+\ldots+r_{t-a_{1}}\right)^{2}+\ldots+C_{m}\left(r_{t-1}+\ldots+r_{t-a_{m}}\right)^{2}+b_{1} \sigma_{t-1}^{2}+\ldots+b_{p} \sigma_{t-p}^{2}
\end{aligned}
$$

onde $\varepsilon_{t} \sim$ i.i.d. $(0,1)$ e $C_{0}>0, \quad C_{j} \geq 0, \quad \forall j=1, \ldots, m-1, \quad C_{m}>0$.

Note-se que usamos letras maiúsculas nos coeficientes do modelo $\operatorname{GHARCH}(m, p)$ parcimonioso, para não confundí-los com os coeficientes do modelo $\operatorname{HARCH}(n)$. Note que se no modelo $\operatorname{GHARCH}(m, p)$ parcimonioso, não consideramos a parte autoregressiva na equação da variância, ou seja, quando $p=0$, o modelo resultante, isto 
é, o modelo $\operatorname{GHARCH}(m)$ parcimonioso, coincide com um modelo $\operatorname{HARCH}(n)$, quando $n=a_{m} \mathrm{e}$

$c_{j}= \begin{cases}C_{i} & j=a_{i} \\ 0 & \text { c.c. }\end{cases}$

Nossa segunda contribuição é o estudo teórico deste novo modelo proposto, ou seja, é a determinação das condições necessárias e suficientes para estacionariedade, ergodicidade, existência de medida invariante, existência do segundo momento etc.

Uma terceira contribuição é a comprovação da estimação de máxima verossimilhança em simulações para validação dos resultados. Para isso foi desenvolvida uma biblioteca em S-Plus, a que deve ser publicada no site do IME-USP posterior à entrega desta tese.

Outra contribuição é a proposta de estimação mediante o amostrador de Griddy-Gibbs, mostrando que os resultados teóricos do amostrador se cumprem na prática, para os exemplos estudados, obtendo-se resultados praticamente iguais aos resultados da estimação por máxima verossimilhança. Devido às características de nossos modelos, uma modificação no algoritmo de Griddy-Gibbs foi proposta para ter uma janela móvel de pontos, para a estimação das condicionais, a cada iteração. As comparações com a estimação de máxima verossimilhança tanto para dados reais quanto para dados simulados foi muito satisfatória. No entanto, a lentidão do algoritmo de Griddy-Gibbs é ainda um problema.

Uma quinta contribuição é a aplicação destes processos a dados reais do Brasil (Ibovespa) e do Euro-Dólar, e a comparação com os processos GARCH, os quais não são consistentes com os resultados teóricos de agregação temporal.

Por último, como uma sexta contribuição, mostramos a utilização destes modelos para previsões da volatilidade, e como seu uso pode contribuir na medição do risco e oportunidades de trading.

A estrutura da tese é a seguinte.

No capítulo 1 apresentamos uma introdução aos dados de alta freqüência e as principais técnicas de modelagem da volatilidade, assim como também os principais modelos de negociação, trading models. Também apresentamos os principais resultados teóricos sob cadeias de Markov e que serão usados nos capítulos posteriores para o estudo teórico do modelo $\operatorname{GHARCH}(m, p)$ parcimonioso proposto.

No capítulo 2 realizaremos o estudo da agregação temporal, também olharemos a modelagem GARCH para dados de alta frequiência e veremos quais são as desvantagens destes modelos e o porquê da necessidade de realizar correções como a adequação do modelo GARCH para que tome em consideração a heterogeneidade da chegada da informação. $\mathrm{O}$ estudo dos fatos estilizados das séries de alta frequiência e aplicações para a taxa de câmbio Euro-Dólar e o índice Ibovespa serão feitas para validar os comentários.

No capítulo 3 apresentamos novas propostas de modelos HARCH e o desenvolvimento teórico destas. Para isso usamos a teoria de cadeias de Markov dada no capítulo 1. Os resultados baseiam-se no estudo das $T$-cadeias. Os critérios para estacionariedade são mostrados. 
No capítulo 4 apresentamos simulações do modelo $\mathrm{HARCH}(m)$ parcimonioso com $m$ agregações. Também veremos o desempenho da estimação de máxima verossimilhança para este tipo de modelos. Devido ao fato da complexidade da função de verossimilhança, dispor de técnicas alternativas pode ser de grande ajuda, assim o amostrador de Griddy-Gibbs é testado. Também realizamos aplicações para a taxa de câmbio Euro-Dólar e o índice Ibovespa. Idéias sobre a avaliação do risco e o uso de processos HARCH para negociação são também mostrados.

Por último, no capítulo 5, são dadas as principais conclusões e quais são as vantagens e desvantagens dos modelos estudados. Também propomos novas extensões que poderiam ser realizadas em trabalhos posteriores.

Devido à importância de validação dos resultados teóricos, vários códigos foram desenvolvidos em S-Plus, esta é uma parte importante e que valida o estudo feito ao longo desta tese. Assim mesmo foram usadas duas séries de alta frequiência, uma com a característica de ser o ativo do mundo mais negociado, Euro-Dólar, e outra por ser uma série de nosso meio, embora seja pouco liquida e tenha muito risco, mas é isso mesmo que valida o estudo, o fato de ter duas séries completamente diferentes, uma de moedas e outra de bolsa. 


\section{Capítulo 1}

\section{Preliminares}

\subsection{Dados de Alta Freqüência.}

A seguir vamos indicar as principais características e utilidades dos dados de alta frequiência assim como as idéias que motivaram o surgimento deste novo tipo de modelagem, sempre dando um enfoque ao ambiente financeiro.

Pelos recentes avanços computacionais, tanto em velocidade de processamento como na capacidade de armazenamento, torna-se cada vez mais factível o uso deste tipo de informação.

Assim, tanto por estes avanços computacionais, quanto pelas técnicas de aquisição de dados, o tipo de informação "on-line" é cada vez maior e mais freqüente no mercado financeiro, e em diversas outras áreas. Este tipo de dados traz consigo informação sobre a microestrutura destes mercados.

Tendo-se dados de alta freqüência muitas propriedades da microestrutura dos mercados financeiros podem ser analisadas e modeladas o que pode ajudar na negociação de ativos financeiros ou na avaliação do risco dos ativos.

No mercado financeiro, existem grandes movimentos dos preços dos ativos em intervalos menores do que um dia. Este fato torna indispensável a modelagem desta informação, pois traz consigo grandes oportunidades para negociar estes ativos.

No entanto, este tipo de informação traz, também, consigo alguns desafios como a filtragem de dados, pois a qualidade destes tem um papel primordial, e o desenvolvimento de novos algoritmos que não somente ajudem a modelar o comportamento destes dados, mas também sejam capazes de trabalhar com grandes volumes e com grande nível de precisão e velocidade. 
O principal desafio das tesourarias dos bancos assim como também das áreas quantitativas, é encontrar os momentos ótimos para "trading". Para isso existem alguns tipos de modelos como os de "pairs trading", "trend following", etc. que ajudam a encontrar oportunidades de "trading".

Em câmbio, nas áreas de risco, o principal desafio está relacionado com a correta avaliação do VaR (Valor em Risco), e portanto, da volatilidade, dos ativos financeiros. Por isso, se tivermos informações mais ricas, que sejam medidas numa freqüência alta, poderíamos entender também o comportamento destes mesmos dados numa freqüência mais baixa, ou ao menos isso parece ter sentido lógico, pois estes dados de baixa frequiência fazem parte da informação dos dados de alta freqüência. Em outras palavras, dados de uma série a cada 5 minutos (frequiência alta), por exemplo, são mais ricos e contêm mais informação que dados da mesma série a cada dia (freqüência baixa). Isto quer dizer que poderíamos ter uma melhoria significativa quanto à previsão destes dados de baixa freqüência.

Em outras palavras, queremos considerar a estrutura do dinamismo do mercado financeiro, para que seja refletido na volatilidade estimada por nossos modelos, para assim aumentar nosso poder de predição.

\subsection{Volatilidade e Tendências.}

Durante os últimos anos foram desenvolvidas diferentes técnicas que possam explicar e modelar os retornos de uma série financeira. No entanto, o comportamento desses retornos parece ser muito complexo e imprevisível.

Isto também é confirmado pelos fatos estilizados das séries financeiras que indicam, entre outras coisas, ausência ou pequena correlação serial da série dos retornos.

Para tentar solucionar este problema, e descrever ao menos, parte do comportamento dos retornos surgem, então, algumas perguntas:

i. Qual é o risco associado ao ativo financeiro? Para isso é necessário modelar a variância ou a volatilidade do ativo financeiro (volatilidade é o desvio padrão da série dos retornos do ativo financeiro).

ii. Esta volatilidade afeta o comportamento dos retornos?

iii. Podemos realizar previsões dos retornos? Se não, que informação podemos obter destes?

iv. A série é persistente (tem memória longa)?

v. A série possui tendência?

vi. Qual é a probabilidade de manter esta tendência?

A segunda pergunta pode ser evidenciada por outros fatos estilizados interessantes: o agrupamento da volatilidade em diferentes níveis, a distribuição não condicional dos retornos com caudas pesadas com relação à distribuição normal, a qual poderia também ajudar a confirmar a memória longa de uma série. 
A maior parte dos modelos estatísticos propostos na literatura são para séries de tempo igualmente espaçadas, isto é trabalhamos com uma série de tempo homogênea. No entanto, uma das alternativas para poder solucionar este inconveniente é considerar os modelos de volatilidade em tempo contínuo, ou então, trabalhar com uma deformação do tempo.

Nós consideraremos $t$ como o índice de uma série de tempo homogênea. Homogênea significa igualmente espaçada para qualquer escala de tempo escolhida.

Alguns modelos para a volatilidade $\sigma_{t}$ são discutidos a seguir.

\subsubsection{Modelos tipo ARCH.}

Estes modelos autoregressivos com heteroscedasticidade condicional definem a variância $\sigma_{t}^{2}$ dos retornos $r_{t}$ como uma função dos retornos passados. Por exemplo, o $\operatorname{ARCH}(1)$ é:

$$
\begin{aligned}
& r_{t}=\sigma_{t} \varepsilon_{t}, \quad \varepsilon_{t} \sim \operatorname{iid}(0,1), \\
& \sigma_{t}^{2}=\alpha_{0}+\alpha_{1} r_{t-1}^{2}
\end{aligned}
$$

Aqui $\varepsilon_{t}$ podem ser normais, $t$-Student ou então alguma distribuição assimétrica ou com caudas pesadas, $\alpha_{0}>0, \alpha_{1} \geq 0$.

Por outro lado nos processos GARCH, a volatilidade $\sigma_{t}$ também depende de seus próprios valores passados, por exemplo o $\operatorname{GARCH}(1,1)$ é:

$$
\begin{aligned}
& r_{t}=\sigma_{t} \varepsilon_{t}, \quad \varepsilon_{t} \sim \operatorname{iid}(0,1) \\
& \sigma_{t}^{2}=\alpha_{0}+\alpha_{1} r_{t-1}^{2}+\beta_{1} \sigma_{t-1}^{2}
\end{aligned}
$$

$\operatorname{com} \alpha_{0}>0, \alpha_{1} \geq 0, \beta_{1} \geq 0, \alpha_{1}+\beta_{1}<1$.

Existem muitas variantes deste tipo de modelos, algumas delas são: GARCH IN MEAN (Bollerslev, 1986), LGARCH (Taylor, 1986), MGARCH (Geweke e Pantula, 1986), IGARCH (Engle e Bollerslev, 1986), EGARCH (Nelson, 1991), TS-GARCH (Schwert, 1989), T-GARCH (Gourieroux, 1992 e Zakonian, 1994), A-PARCH (Ding et. al., 1993), Q-GARCH (Sentana, 1991), HARCH (Müller et. al., 1997) etc. 


\subsubsection{Modelos de Volatilidade Estocástica (MVE).}

Nestes, além de um ruído branco na equação dos retornos, temos um outro ruído na equação da variância, isto é, a volatilidade não é observável nem calculável diretamente a partir dos retornos passados. Uma diferença importante com os modelos $\mathrm{ARCH}$ é que aqui podemos analisar MVE com tempo contínuo.

Existem muitas formas de modelo de volatilidade estocástica, sendo a mais típica delas a:

$$
\begin{aligned}
& r_{t}=\varepsilon_{t} \exp \left(\frac{h_{t}}{2}\right), \quad \varepsilon_{t} \sim \operatorname{iid}(0,1), \\
& h_{t}=\alpha_{0}+\alpha_{1} h_{t-1}+\eta_{t},
\end{aligned}
$$

onde $\eta_{t}$ é uma série estacionária Gaussiana, com média 0 e variância $\sigma_{\eta}^{2}$, sendo $\eta_{t} \perp \varepsilon_{t}$.

\subsubsection{Modelos baseados na Volatilidade Realizada.}

Em lugar de modelar a volatilidade $\sigma_{t}$, define-se a volatilidade realizada $\sigma_{t}^{\text {realizada }}$ como o desvio padrão (mais geralmente como uma função) dos retornos no tempo $t$ - $1 \mathrm{e}$ com os dados dos retornos numa maior freqüência, e depois transformada à menor freqüência.

Isto é, por exemplo, a volatilidade realizada para o "dia" de hoje, é calculada como o desvio padrão dos retornos (horários, por exemplo) de ontem e multiplicado por $\sqrt{n}$, donde $n$ é o número de retornos usados para o cálculo do desvio padrão.

Embora este modelo tenha a vantagem de usar dados empíricos em lugar de fazer suposições no modelo que poderiam ser errôneas, têm algumas desvantagens como a volatilidade ser viciada e defasada, o que conduz a previsões sub-ótimas da volatilidade, além de que a correlação dos retornos poderia afetar o cálculo desta. Um método de correção de vício da volatilidade dinâmica realizada como o dado por Dacorogna et al. (2001) poderia melhorar o modelo.

\subsubsection{Modelos de Volatilidade Histórica.}

Aqui a volatilidade é calculada a partir de uma série de tempo $\sigma_{t}$ de volatilidades realizadas. 
A principal vantagem que existe, é que similarmente aos modelos de volatilidade realizada, usam-se os dados empíricos para construir a série.

\subsubsection{Modelos de Volatilidade Implícita.}

Nestes modelos, a volatilidade é calculada a partir da equação de Black and Scholes, uma vez que no mercado financeiro se dispõe dos preços das opções dos ativos. Para mais detalhes veja Hull (1995).

$\mathrm{Na}$ maioria destes modelos, para a estimação da volatilidade $\sigma_{t}^{2}$, é possível incluir termos para modelar as influências de variáveis externas (exógenas).

Agora vamos responder às outras perguntas que fizemos no inicio desta seção, isto é: a série é persistente (tem memória longa)? A série possui tendência? Qual é a probabilidade de manter esta tendência? Para isso são necessários modelos como aqueles descritos a seguir.

\subsubsection{Trend Following.}

Estes modelos tentam acompanhar a tendência de uma série, tentando descobrir o mais breve possível o início e o final desta tendência. Os principais modelos são: Moving Average Convergence Divergence (MACD), Parabolic Systems, Modelos de médias móveis exponenciais (EWMA), Modelos de Stopping Time Value, Modelos Não Paramétricos, Modelos de Redes Neurais etc. Alguns detalhes podem ser vistos em Duda et al. (2001), Theodoridis e Koutroumbas (2006) e Dunis et al. (2003).

Estes tipos de modelos são principalmente úteis em séries com persistência ou memória longa e não em séries caóticas. Para isso são necessários testes de memória longa ou modelos que ajudem a medir o caos da série como o expoente de Hurst ou modelos de entropia. Então, quando uma série apresente memória longa, ou em outras palavras, pouca caoticidade, ou níveis baixos de entropia, podemos, então aplicar à série, durante o tempo que apresente estas características, estes modelos de trend following.

\subsubsection{Outros Trading Models.}

O objetivo é indicar o momento adequado para realizar um "trade" numa série analisada. Isto é importante no ambiente financeiro pois este conhecimento é fundamental nas operações das tesourarias dos bancos.

Modelos que ajudam neste sentido são os de "pairs trading", que tratam técnicas como a cointegração para determinar sobreprecificação de ativos; exemplos podem ser vistos em Vidyamurthy (2004), reconhecimento de padrões ou sinais, estes modelos ajudam 
a descobrir mudança de direção mudanças estruturais, de regime, e abruptas. Neste contexto entram modelos de sobrevivência, de redes neurais, inteligência artificial, ou modelos não paramétricos como vizinhos mais próximos, análise de Cluster, cadeias ocultas de Markov etc. Mais detalhes encontram-se em Theodoridis e Koutroumbas (2006) e em Duda et al. (2001). Por último existem várias "trading rules", muitas delas sem bases teóricas, mas que de alguma forma são usadas no âmbito financeiro, para mais detalhes sobre estas e outras técnicas veja Park e Irwin (2005), Acar e Satchell (2002) e Dunis et al. (2003).

Algumas séries financeiras são influenciadas pelo comportamento de outras. Assim, testes de causalidade para influência de variáveis externas dentro de um modelo são também importantes.

Devido ao fato de que o tempo físico não necessariamente evolui igual ao tempo de negócios, modelos de deformação temporal podem ser úteis na explicação do comportamento de uma série financeira.

Para poder demonstrar algumas condições sobre os parâmetros destes tipos de modelagem, para obter propriedades como, por exemplo, estacionariedade, às vezes é necessário expressar nosso modelo em termos de cadeias de Markov, para isso vamos mencionar algumas definições e resultados sobre cadeias de Markov, que usaremos depois.

\subsection{Alguns Resultados sobre Cadeias de Markov.}

Alguns destes resultados podem ser vistos em detalhe em Meyn e Tweedie (1996).

Considere-se a coleção de variáveis aleatórias

$$
\mathbf{X}=\left\{X_{n}: n \in \mathbb{Z}^{+}\right\}, \mathbb{Z}^{+}:=\{0,1, \ldots\},
$$

definidas sobre $(\Omega, \mathfrak{I})$. Dizemos que o processo $\mathbf{X}$ tem a propriedade Markoviana se o futuro do processo depende somente de seu valor presente e independe de seu passado. Em outras palavras, um processo que tem a propriedade Markoviana seria um processo com perda de memória.

Note-se que se $\mathbf{Y}=\left\{Y_{n}\right\}$ é um processo com memória finita, $\mathrm{k}$, então o processo $\mathbf{X}=\left\{X_{n}\right\}$, com $X_{n}=\left\{Y_{n}, \ldots Y_{n-k+1}\right\}$ possui a propriedade Markoviana.

Assumimos que $\Omega$ é equipado com uma topologia mensurável, separável, localmente compacta, sendo $B(\Omega)$ a sigma álgebra de Borel.

Seja $P$ a lei de probabilidade sobre a cadeia de Markov de tempo homogêneo $\mathbf{X}$, então temos que:

$$
P\left(X_{n+m} \in A \mid X_{j}, j \leq m ; X_{m}=x\right)=P^{n}(x, A),
$$


onde as probabilidades de transição $\left\{P^{n}(x, A), x \in \Omega, A \subset \Omega\right\}$ denotam a probabilidade da cadeia, estando no estado $x$, passe a estar no conjunto $A$ depois de $n$ passos ou transições.

A independência de $X_{n+m}$ com relação aos valores $X_{j}, j<m$, refletida por (1.1) é a propriedade Markoviana.

\section{Definição 1.1.}

Se $\left\{P^{n}(x, A), x \in \Omega, A \subset \Omega\right\}$ é tal que:

$\forall A \in B(\Omega), P(\bullet, A)$ é uma função não negativa mensurável sob $\Omega$,

$\forall x \in \Omega, P(x, \bullet)$ é uma medida de probabilidade sob $B(\Omega)$, então $P$ é uma probabilidade de transição.

\section{Definição 1.2.}

Sejam $\mathbf{X}=\left\{X_{0}, X_{1}, \ldots, X_{n}\right\}$ variáveis aleatórias com

$$
\mathbf{X} \in \mathbf{\Omega}=\prod_{i=0}^{n} \Omega_{i}, X_{i} \in \Omega_{i}
$$

e com uma $\sigma$-álgebra, $\mathfrak{I}$, adequada.

O processo $\mathbf{X}$ é chamado cadeia de Markov de tempo homogêneo, com probabilidade de transição $P(x, A)$ e distribuição inicial $v$ se a distribuição finita de $\mathbf{X}$ satisfaz, $\forall n$

$$
P\left(X_{0} \in A_{0}, \ldots, X_{n} \in A_{n}\right)=\int_{y_{0} \in A_{0}} \ldots \int_{y_{n-1} \in A_{n-1}} v\left(d y_{0}\right) P\left(y_{0}, d y_{1}\right) \ldots P\left(y_{n-1}, A_{n}\right)
$$

\section{Definição 1.3.}

Uma função $f: \Omega \rightarrow \mathbb{R}$ é chamada de menor função semicontínua se $\liminf _{y \rightarrow x} f(y) \geq f(x), x \in \Omega$. 


\section{Definição 1.4.}

Se a matriz ${ }^{2}$ de transição $P(\cdot, O)$ é a menor função semicontínua para qualquer conjunto aberto $O \in B(\Omega)$, então $P$ é chamada de cadeia fraca de Feller.

\section{Definição 1.5.}

Uma cadeia $\mathbf{X}$ é chamada de $\varphi$-irredutível se existe uma medida $\varphi$ sobre $B(\Omega)$, tal que $\forall x \in \Omega$, sempre que $\varphi(A)>0$, tem se $U(x, A):=\sum_{n=1}^{\infty} P^{n}(x, A)>0$.

\section{Definição 1.6.}

A medida $\psi$ é dita maximal com respeito a $\varphi, \psi \succ \varphi$, se $\psi(A)=0 \Rightarrow \varphi(A)=0$.

\section{Teorema 1.1.}

Se $\mathbf{X}$ é $\varphi$-irredutível, então existe uma medida de probabilidade $\psi \in B(\Omega)$ tal que $\mathbf{X}$ é $\psi$-irredutível.

Para qualquer outra medida $\varphi^{\prime}$, a cadeia é $\varphi^{\prime}$-irredutível se e somente se $\psi \succ \varphi^{\prime}$. Se $\psi(A)=0$, então $\psi\{y: U(y, A)>0\}=0$.

A medida de probabilidade $\psi$ é equivalente a

$$
\psi^{\prime}(A):=\int_{\Omega} \varphi^{\prime}(d y) K_{a_{\frac{1}{2}}}(y, A)
$$

com

$$
K_{\frac{a_{1}}{2}}(y, A):=\sum_{n=0}^{\infty} P^{n}(y, A) 2^{-(n+1)}, y \in \Omega, A \in B(\Omega)
$$

\footnotetext{
${ }^{2}$ A terminologia de matriz vem das cadeias de Markov de espaço de estados discretos.
} 


\section{Notação 1.1.}

Dizemos que uma cadeia de Markov é $\psi$-irredutível se é $\varphi$-irredutível para alguma medida $\varphi$ e a medida $\psi$ é uma medida de irredutibilidade maximal satisfazendo as condições do teorema anterior.

Seja $d=\{d(n)\}$ uma distribuição ou medida de probabilidade sobre $\mathbb{Z}^{+}$e consideremos a cadeia de Markov $\mathbf{X}_{d}$ com kernel de transição:

$$
K_{d}(x, A):=\sum_{n=0}^{\infty} P^{n}(x, A) d(n), x \in \Omega, A \in B(\Omega) .
$$

\section{Definição 1.7.}

Se $d$ é uma distribuição amostral e existe um kernel de transição subestocástico $T$ que satisfaz:

$$
K_{d}(x, A) \geq T(x, A), x \in \Omega, A \in B(\Omega)
$$

onde $T(\cdot, A)$ é a menor função semicontínua para qualquer $A \in B(\Omega)$, então $T$ é chamada de componente contínua de $K_{d}$.

\section{Definição 1.8.}

Se $\mathbf{X}$ é uma cadeia de Markov para a qual existe uma distribuição amostral $d$ tal que $K_{d}$ possui uma componente contínua $T, \operatorname{com} T(x, \Omega)>0, \forall x$, então $\mathbf{X}$ é chamada uma $T$-cadeia.

\section{Teorema 1.2.}

Se $\mathbf{X}$ é uma cadeia de Feller $\psi$-irredutível tal que supp $\psi$ tem interior não vazio, então $\mathbf{X}$ é uma $T$-cadeia $\psi$-irredutível. 


\section{Definição 1.9.}

Chamaremos um conjunto $C \in B(\Omega) \quad v_{a}$-petite se a cadeia amostrada satisfaz a seguinte condição:

$$
K_{a}(x, A) \geq v_{a}(A), \forall x \in C, A \in B(\Omega),
$$

onde $v_{a}$ é uma medida não trivial sobre $B(\Omega)$.

\section{Teorema 1.3.}

Se $\mathbf{X}$ é uma $T$-cadeia $\psi$-irredutível então todo conjunto compacto é petite.

\section{Definição 1.10.}

Chamaremos uma função $V: \Omega \rightarrow \mathbb{R}^{+}$"unbounded off petite" para $\mathbf{X}$, se para qualquer $n<\infty$, o conjunto

$$
C_{V}(n)=\{y: V(y) \leq n\}
$$

é petite.

\section{Definição 1.11.}

A cadeia $\mathbf{X}$ é chamada recorrente se é $\psi$-irredutível e se

$$
U(x, A) \equiv \infty, \forall x \in \Omega \text { e } \forall A \in B(\Omega)
$$

$\operatorname{com} \psi(A)>0$.

As cadeias de Markov recorrentes são importantes porque nos ajudam a identificar se um estado ou conjunto de estados é passageiro ou pode ser alcançado um número finito de vezes. Neste caso estamos falando que esse estado ou conjunto de estados é transitório. Em outras palavras, numa cadeia de Markov recorrente o número esperado de visitas a um conjunto de estados, $A$, de medida não nula, desde um ponto inicial $x$, em $n$ passos, é infinito. 


\section{Definição 1.12.}

O conceito de periodicidade, definido em cadeias de Markov sobre espaço de estados enumerável, pode ser também estendido para espaço de estados não enumerável para cadeias $\psi$-irredutíveis, simplesmente dividindo o espaço de estados em conjuntos

disjuntos, assim $D_{i} \subset B(\Omega), \forall i=1, \ldots, p, \operatorname{com} \bigcup_{i=1}^{p} D_{i}=\Omega$ e $\bigcap D_{i} D_{j}=\varnothing$, $\forall i, j=1, \ldots, p, i \neq j$.

Para ver se uma cadeia de Markov é periódica selecionamos todos os conjuntos disjuntos $D^{(i)}=\left\{D_{1}^{(i)}, \ldots, D_{p^{(i)}}^{(i)}\right\}$ de tamanho $p^{(i)}$ tal que $P\left(x, D_{j+1}^{(i)}\right)=1, \forall x \in D_{j}^{(i)}$, $j=1, \ldots, d(\bmod d)$.

Então dizemos que a cadeia de Markov $\mathbf{X}$ é periódica com período $p$ igual ao m.d.c. $\operatorname{dos} p^{(i)}$. Se $p=1$, então $\mathbf{X}$ é chamada de cadeia aperiódica. E se além disso existe um conjunto $A$ tal que $\forall x \in A, \forall B \in B(\Omega), P(x, B)>0$, então a cadeia é chamada de fortemente aperiódica.

\section{Definição 1.13.}

Seja $\mathbf{X}$ uma cadeia de Markov $\psi$-irredutível.

Chamaremos o conjunto $A \in B(\Omega)$ total se $\psi\left(A^{c}\right)=0$.

Chamaremos o conjunto $A \in B(\Omega)$ absorvente se $P(x, A)=1, x \in A$.

Pode ser demonstrado que uma cadeia de Markov $\psi$-irredutível é ou transitória ou recorrente.

O nosso objetivo será identificar cadeias de Markov recorrentes e para isso vamos mencionar alguns teoremas que usaremos mais adiante.

\section{Teorema 1.4.}

Suponhamos que $\mathbf{X}$ seja uma cadeia $\psi$-irredutível.

Se existe um conjunto petite $C \in B(\Omega)$ e uma função positiva $V$, a qual é unbounded off petite, tal que

$$
V\left(X_{n}\right)-E\left[V\left(X_{n+1}\right) \mid X_{n}\right] \geq 0, X_{n} \in C^{c}
$$

então, a cadeia de Markov $\mathbf{X}$ é recorrente. 
Outra propriedade interessante das cadeias de Markov tem a ver com a nãoevanescência. Isto é, se a cadeia de Markov caminha para o infinito ou não. Para formalizar isto é necessário, primeiramente, dar umas definições.

\section{Definição 1.14.}

Chamaremos de pré-compacto um conjunto $P$ tal que o fecho de $P$ é compacto.

\section{Definição 1.15.}

Diremos que a cadeia de Markov visita o conjunto $A$ infinitas vezes, e denotaremos por $\{X \in A \quad$ i.o. $\}$, ou por $\eta_{A}=\infty$, se

$$
\{X \in A \quad \text { i.o. }\}:=\bigcap_{N=1}^{\infty} \bigcup_{k=N}^{\infty}\left\{X_{k} \in A\right\}
$$

Agora, diremos que uma cadeia de Markov vai infinitas vezes ao infinito se o conjunto $\{X \rightarrow \infty\}=\bigcap_{n=0}^{\infty}\left\{X \in P_{n} \quad \text { i.o. }\right\}^{c}$, onde $\left\{P_{n}: n \in \mathbb{Z}^{+}\right\}$é uma coleção de conjuntos abertos pré-compactos.

\section{Definição 1.16.}

Uma cadeia de Markov $\mathbf{X}$ é chamada de não-evanescente se $P[x,\{X \rightarrow \infty\}]=0$, $\forall x \in \Omega$.

\section{Teorema 1.5.}

Suponhamos que $\mathbf{X}$ seja uma cadeia de Markov e que existe uma função positiva $V$, com $V(x) \rightarrow \infty$ quando $x \rightarrow \infty$, e tal que

$$
V\left(X_{n}\right)-E\left[V\left(X_{n+1}\right) \mid X_{n}\right] \geq 0, X_{n} \in C^{c}
$$

onde $C \in B(\Omega)$ é um conjunto compacto. Então $X$ é não-evanescente. 


\section{Definição 1.17.}

Uma $T$-cadeia, $\psi$-irredutível e não-evanescente é chamada de Harris recorrente.

\section{Definição 1.18.}

Uma medida $\pi$ sob $B(\Omega), \sigma$-finita, com a propriedade

$$
\pi(A)=\int_{\Omega} \pi(d x) P(x, A), A \in B(\Omega)
$$

será chamada medida invariante.

Se $\mathbf{X}$ é $\psi$-irredutível e admite uma medida de probabilidade invariante, $\pi$, então $\mathbf{X}$ é chamada cadeia positiva.

Se $\mathbf{X}$ não admite uma medida invariante, então $\mathbf{X}$ é chamada nula.

Se $\mathbf{X}$ é Harris recorrente e positiva, então a cadeia $\mathbf{X}$ é chamada Harris positiva.

\section{Teorema 1.6.}

Suponhamos que $\mathbf{X}$ seja uma cadeia de Feller e que existe uma função positiva $V$ tal que

$$
V\left(X_{n}\right)-E\left[V\left(X_{n+1}\right) \mid X_{n}\right] \geq 0, X_{n} \in C^{c}
$$

onde $C$ é um conjunto compacto. Então existe uma medida invariante, finita sobre subconjuntos compactos de $\Omega$.

Os teoremas (1.4), (1.5) e (1.6) dão a condição que é conhecida como critério de recorrência. Agora teremos vários teoremas que dão uma segunda condição que é também conhecida como critério de regularidade e positividade ou também como condição de Foster.

\section{Definição 1.19.}

Um conjunto $C \in B(\Omega)$ é chamado regular quando $\mathbf{X}$ é $\psi$-irredutível e

$$
\sup _{x \in C} E_{x}\left[\tau_{B}\right]<\infty, \forall B \in B(\Omega), \psi(B)>0,
$$


onde $\tau_{B}=\min \left\{n \geq 1: X_{n} \in B\right\}$ que é conhecido como o primeiro tempo de retorno ao conjunto $B$, e $x$ é o ponto de partida.

Em outras palavras, um conjunto $B$ é chamado regular se partindo desde qualquer ponto dele podemos alcançar qualquer conjunto Boreliano, de medida positiva, em um tempo finito.

Uma cadeia $\mathbf{X}$ é chamada regular se existe uma cobertura contável de $\Omega$ por conjuntos regulares.

\section{Teorema 1.7.}

Suponhamos que $C \in B(\Omega)$, sendo $C$ um conjunto petite, e que existe uma função $V: \Omega \rightarrow[0, \infty)$, e uma constante $b<\infty$, tal que

$$
V\left(X_{n}\right)-E\left[V\left(X_{n+1}\right) \mid X_{n}\right] \geq 1-b 1_{C}\left(X_{n}\right), X_{n} \in \Omega
$$

Então:

(i) Se $V$ finita em qualquer lugar e limitada sobre $C$, então $\mathbf{X}$ é Harris positiva. Também tem-se para cadeias $\psi$-irredutíveis que:

(ii) Se $V$ é limitado sobre A, então A é regular.

(iii) $\mathrm{Se} \mathrm{V}$ finita em qualquer lugar, então $\mathbf{X}$ é regular.

\section{Teorema 1.8 .}

Suponhamos que $\mathbf{X}$ seja uma cadeia fraca de Feller. Sejam $C \in B(\Omega)$, sendo $C$ um conjunto compacto, e $V$ uma função positiva finita em algum $x_{0} \in \Omega$. Se

$$
V\left(X_{n}\right)-E\left[V\left(X_{n+1}\right) \mid X_{n}\right] \geq 1-b 1_{C}\left(X_{n}\right), X_{n} \in \Omega
$$

sendo $b$ uma constante, $b<\infty$, então existe uma medida de probabilidade invariante $\pi$.

\section{Teorema 1.9. Ergodicidade Aperiódica}

Suponhamos que $\mathbf{X}$ seja uma cadeia Harris recorrente aperiódica, com medida invariante $\pi$. 
Dizer que a cadeia é Harris positiva ${ }^{3}$ é equivalente a dizer que existe um conjunto petite $C$, algum $b<\infty$ e uma função não negativa $V$ finita em um $x_{0} \in \Omega$, satisfazendo

$$
V\left(X_{n}\right)-E\left[V\left(X_{n+1}\right) \mid X_{n}\right] \geq 1-b 1_{C}\left(X_{n}\right), X_{n} \in \Omega \text {. }
$$

Agora vamos mencionar vários teoremas que nos vão dar uma nova condição para obter $f$-positividade e $f$-regularidade. Estes teoremas podem servir, por exemplo, para encontrar as condições para a existência dos momentos de ordem maior ou igual a 2 da distribuição estacionária.

\section{Definição 1.20.}

Diremos que uma cadeia de Markov, $\mathbf{X}$, é f-ergódica se $f \geq 1$ e:

1. $\mathbf{X}$ é Harris recorrente positiva com probabilidade invariante $\pi$.

2. O valor esperado $\pi(f):=\int \pi(d x) f(x)$ é finito.

3. Para cada condição inicial da cadeia,

$$
\lim _{k \rightarrow \infty}\left\|P^{k}(x, \bullet)-\pi\right\|_{f}=0
$$

\section{Definição 1.21.}

Um conjunto $C \in B(\Omega)$ é chamado $f$-regular, onde $f: \Omega \rightarrow[1, \infty)$ é uma função mensurável, se para cada $B$ com medida positiva, $B \in B(\Omega)$,

$$
\sup _{x \in C} E_{x}\left[\sum_{k=0}^{\tau_{B}-1} f\left(X_{k}\right)\right]<\infty .
$$

A cadeia $\mathbf{X}$ é chamada $f$-regular se existe uma cobertura contável de $\Omega$ com conjuntos $f$-regulares.

\footnotetext{
${ }^{3}$ Se uma cadeia for Harris positiva, a única medida invariante é finita.
} 


\section{Teorema 1.10.}

Suponhamos que $\mathbf{X}$ seja uma cadeia $\psi$-irredutível e aperiódica, então as seguintes condições são equivalentes:

(i) Existe uma função $f: \Omega \rightarrow[1, \infty)$, um conjunto $C \in B(\Omega)$, uma constante $b<\infty$, e uma função $V: \Omega \rightarrow[0, \infty)$, tais que

$$
V\left(X_{n}\right)-E\left[V\left(X_{n+1}\right) \mid X_{n}\right] \geq f\left(X_{n}\right)-b 1_{C}\left(X_{n}\right), X_{n} \in \Omega
$$

(ii) A cadeia é positiva recorrente com medida de probabilidade invariante $\pi$, com $\pi(f)<\infty$.

\section{Teorema 1.11.}

Suponhamos que $\mathbf{X}$ seja uma cadeia $\psi$-irredutível.

Se para uma função $f: \Omega \rightarrow[1, \infty)$, um conjunto $C \in B(\Omega)$, uma constante $b<\infty$, e uma função $V: \Omega \rightarrow[0, \infty)$, tivermos

$$
V\left(X_{n}\right)-E\left[V\left(X_{n+1}\right) \mid X_{n}\right] \geq f\left(X_{n}\right)-b 1_{C}\left(X_{n}\right), X_{n} \in \Omega,
$$

então temos:

(i) Se $\mathrm{C}$ é petite e $\mathrm{V}$ é limitada sobre um conjunto $\mathrm{A}$, A é f-regular.

(ii) A cadeia é $f$-regular se e somente se a função $V$ é finita em qualquer lugar. $\square$

\section{Teorema 1.12.}

Suponhamos que $\mathbf{X}$ seja uma cadeia recorrente positiva e aperiódica.

Se $\mathbf{X}$ é $f$-regular, então $\mathbf{X}$ é $f$-ergódica.

Inversamente, se $\mathbf{X}$ é $f$-ergódica, então $\mathbf{X}$, restrita a um conjunto totalmente absorvente, é $f$-regular. 


\section{Capítulo 2}

\section{Modelagem de Dados de Alta Freqüiência}

\subsection{Introdução.}

Como comentado anteriormente, existem muitas variantes dos modelos $\mathrm{ARCH}$ de Engle. Dentro estas variantes estão os modelos HARCH que consideram o comportamento heterogêneo das séries financeiras. Este tipo de modelagem parece ser mais adequado ao estudo de dados de alta freqüência.

Quando falamos de comportamento heterogêneo nos referimos à chegada da informação ao mercado financeiro, a qual é heterogênea e aproveitada de diferentes maneiras pelos diversos tipos de agentes do mercado.

Assim, para os "market makers" e quem trabalha arbitragem, informação de muito curto tempo (ao redor de uma hora) os leva a agir de uma certa forma sob a variável financeira analisada. Por outro lado, existem traders intraday, de curto prazo, de médio prazo e de longo prazo. Todos estes agindo de formas diferentes com relação à chegada de informações.

Por último, podemos citar aos grandes investidores, que têm um período muito maior para a tomada de decisões. 
Estes são só alguns exemplos que podemos citar, de diferentes perfis de pessoas, agindo diretamente no comportamento de uma série financeira e influenciando diretamente nos agrupamentos da volatilidade.

Antes de descrever os processos HARCH é importante primeiro entender algumas características dos processos da família ARCH.

\subsection{Modelos GARCH.}

Os modelos ARCH de Engle (1982) e GARCH de Bollerslev (1986) são os mais amplamente usados para a modelagem da volatilidade de variáveis econômicas e financeiras, especialmente dentro do contexto de avaliação do risco. A principal suposição destes modelos é a relativa homogeneidade nos processos de descoberta de preço pelos agentes do mercado.

No entanto vários fatos empíricos estão em conflito com esta visão homogênea do mercado.

Primeiro, pelo comportamento de memória longa que apresentam diversos tipos de séries financeiras, embora esta característica tenha sido resolvida com certo sucesso com a incorporação dos modelos FIGARCH, como pode ser visto em Baillie et al. (1996).

Segundo, pela chegada não homogênea da informação dentro do mercado, que afeta a volatilidade, e implica que os movimentos nas séries de tempo de alta frequiência não sejam necessariamente relacionados com a chegada de novas notícias, pois, por exemplo, os negociantes intradia têm objetivos e comportamentos diferentes que os negociantes de longo prazo.

Terceiro, em dados de alta frequiência, às vezes, as séries de tempo revelam padrões sazonais.

Este comportamento heterogêneo da chegada da informação é um aspecto fundamental que consideram os processos HARCH.

Mesmo, levando em consideração a sazonalidade e a memória longa, os processos GARCH não resolvem o problema da heterogeneidade do mercado. Isto fica mais claro quando se trabalha com dados de alta frequiência, pois este comportamento heterogêneo do mercado fica mais evidente. Isto também pode ser demonstrado teoricamente, quando é estudada a agregação temporal dos processos $\mathrm{GARCH}$, para dados de alta freqüência.

\subsection{Deformação do Tempo Físico.}

Existem alguns fatos importantes que conduzem à necessidade de realizar uma deformação temporal na série de tempo antes desta ser estudada. Nas séries financeiras, por exemplo, a chegada de novas informações ao mercado é importante. Assim, dias em que novidades não surgem, sejam "boas" ou "ruins", são caracterizados 
por pouca movimentação, ao passo que dias carregados de novas informações têm efeito justamente oposto, ou seja, apresentam alta variação nos preços.

Do mesmo modo, temos dias ou horários onde não existe trabalho. Por exemplo, no período de fechamento das bolsas de valores até a próxima abertura, não existe informação, ficando assim o último dado repetido muitas vezes até a seguinte variação ${ }^{4}$ A série assim descrita pode dar uns saltos abruptos, dependendo da informação que chegou ao mercado financeiro neste período. Nos mercados de taxas de cambio, as horas da madrugada dos principais mercados envolvidos, são caracterizadas, também, por pouca movimentação. Diferentemente da bolsa e de outros ativos financeiros, os mercados de $F X$ (taxas de cambio) funcionam 24 horas por dia, 7 dias por semana.

De qualquer modo, esta movimentação dos mercados dá uma aparente sazonalidade nas séries, que deve ser tratada; e uma maneira sugerida é mediante uma deformação temporal.

Note-se que a seleção do tempo físico para a análise das séries não é a melhor, pois traz consigo informação que não é verdadeira, o que pode ocasionar um colapso nas previsões.

Além disso, o fato de tratar uma série que não é homogênea no tempo ${ }^{5}$, como se ela fosse, isto é, igualmente espaçada no tempo físico, quando ela flui de modo diferente (dependendo da movimentação do mercado) pode introduzir informação inexistente, tal como:

1) A ocorrência de correlações seriais significativas na série dos retornos.

2) Existência de correlação cruzada significativa entre duas séries.

3) Existência de correlação serial significativa na série dos retornos de uma carteira.

Isto pode ser visto com mais detalhes em Tsay (2002).

Ao introduzir uma deformação temporal no modelo, o que estamos fazendo é, no lugar de modelar a série como um processo estocástico normal, consideramos processos estocásticos subordinados (Mandelbrot e Taylor, 1967 e Clark, 1973).

Assim, segundo esta abordagem, não olhamos mais para nosso processo de interesse evoluindo através do tempo calendário que é igualmente espaçado, mas sim evoluindo de acordo com um "tempo de negócios"; que pode ser uma função da chegada de uma nova informação, do volume negociado, do dia da semana, e mesmo do tempo físico, entre outras variáveis, originando uma nova dimensão temporal. Alguns resultados para volatilidade estocástica encontram-se em Ghysels e Jasiak (1994).

\footnotetext{
${ }^{4}$ Quando considerado estritamente o tempo físico. Neste caso o valor da série atual, dado que não existiu, é o ultimo valor registrado; repetindo-se assim este valor até a chegada de um novo valor.

${ }^{5}$ Note-se que o conceito de uma série de tempo homogênea é diferente ao da homogeneidade da chegada da informação mencionada anteriormente.
} 
Um modo simples de deformar o tempo é trabalhar com a escala de tempo de negócios. Este tempo de negócios simplesmente omite os períodos de final de semana, sábados e domingos, e em geral quando os mercados são virtualmente fechados ${ }^{6}$.

\subsection{Agregação Temporal.}

Os modelos GARCH tem sido usados com sucesso para modelar séries em diversas frequiências ${ }^{7}$. A idéia da agregação temporal nasce quando intuitivamente assume-se que um processo GARCH, numa determinada frequiência, digamos diária, é consistente ${ }^{8}$ com algum outro processo numa outra freqüência, digamos semanal.

Esta propriedade interessante que apresentam os processos $\mathrm{GARCH}$, a de agregação temporal, pode ser vista em Drost e Nijman (1993) ou Nelson e Foster (1994).

Esta característica diz que se a série temporal é descrita como gerada por um processo GARCH em uma particular frequiência, o comportamento destes dados em qualquer outra frequiência é explicado por um outro processo GARCH que é determinado pela agregação (ou desagregação) temporal do processo original, sob certas hipóteses que descreveremos depois. Em outras palavras, está-se falando que os processos GARCH são fechados, sob agregação temporal.

Isto é, quando falamos de agregação (desagregação) temporal, os parâmetros da equação de variância de um modelo de baixa (alta) freqüência, dependem dos parâmetros da variância e curtose do correspondente modelo de alta (baixa) frequiência. Assim, para ver se um processo GARCH explica o comportamento de uma série temporal, em uma particular frequiência, digamos freqüência 1, podemos derivar teoricamente o processo GARCH que explicaria o comportamento desta série em outra frequiência, digamos de frequiência 2, e compará-lo com o ajuste GARCH da série nesta freqüência 2. Assim, o resultado teórico e do ajuste deveriam ser próximos, validando assim, que o processo GARCH original explica a série temporal na freqüência 1. Têmse alguns resultados nesta direção para freqüências diárias e semanais.

Entretanto, diferenças significativas entre os resultados empíricos e teóricos sugerem à rejeição da hipótese de somente um processo GARCH gerar os dados, podendo mostrar que há mais que uma frequiência relevante na geração da volatilidade, podendo o mercado ser chamado de heterogêneo. E isto é verdade independente da distribuição assumida para o ruído branco. Inclusive, quando é eliminada a sazonalidade mediante uma deformação temporal, este problema persiste.

Um fato importante a considerar é que ao fazer a agregação ou desagregação temporal, altas frequiências podem ser úteis devido à grande significância que é apresentada nos

\footnotetext{
${ }^{6}$ Falamos de virtualmente fechados pois dependem do ativo financeiro a ser tratado, sendo que o mercado de FX não fecha nunca, pode ser considerado fechado naqueles períodos de menor liquidez ou movimentação, geralmente finais de semana, feriados, etc. das zonas de interesse.

${ }^{7}$ Geralmente a partir de frequiências diárias.

${ }^{8}$ Consistente no sentido de que se o processo GARCH explica o comportamento dos dados de alta freqüência, um novo processo derivado deste, fará o mesmo em baixa freqüência.
} 
dados. No entanto, deve-se ter cuidado, porque por este mesmo fato, qualquer desvio, por minúsculo que seja, pode conduzir a rejeição da hipótese nula.

Outro fato interessante, mostrado por Diebold (1988) é que quando fazemos agregação temporal, a heteroscedasticidade condicional desaparece se o intervalo de tempo amostral vai para infinito.

Drost e Nijman (1993) mostraram que os processos $\operatorname{GARCH}(1,1)$ são fechados sob agregação temporal, quando eles são simétricos e fracos.

Para entender melhor o problema de agregação temporal, vamos dar algumas definições:

\section{Definição 2.1.}

Uma série temporal $r_{t}$, estacionária, invertível e com seu quarto momento finito, é gerada por um processo $\operatorname{GARCH}(p, q)$ forte, se os parâmetros deste processo GARCH

podem ser escolhidos tal que $\varepsilon_{t}=r_{t} / \sigma_{t}$ são i.i.d., seguindo uma distribuição $D$ com média zero e variância unitária.

\section{Definição 2.2.}

Uma série temporal $r_{t}$, estacionária, invertível e com seu quarto momento finito, é gerada por um processo $\operatorname{GARCH}(p, q)$ semi-forte, se os parâmetros deste processo GARCH podem ser escolhidos tal que $E\left[r_{t} \mid r_{t-1}, r_{t-2}, \ldots\right]=0 \quad \mathrm{e}$ $E\left[r_{t}^{2} \mid r_{t-1}, r_{t-2}, \ldots\right]=\sigma_{t}^{2}$ (diferença martingale).

\section{Definição 2.3.}

Uma série temporal $r_{t}$, estacionária, invertível e com seu quarto momento finito, é gerada por um processo $\operatorname{GARCH}(p, q)$ fraco, se os parâmetros deste processo GARCH podem ser escolhidos tal que

$$
P\left[r_{t} \mid r_{t-1}, r_{t-2}, \ldots\right]=0 \text {, e } P\left[r_{t}^{2} \mid r_{t-1}, r_{t-2}, \ldots\right]=\sigma_{t}^{2} \text {. }
$$


Aqui, $P\left[\chi_{t} \mid r_{t-1}, r_{t-2}, \ldots\right]=0$ denota o melhor preditor linear de $\chi_{t}$ em termos de $1, r_{t-1}, r_{t-2}, \ldots, r_{t-1}^{2}, r_{t-2}^{2} \ldots$, isto é, $E\left(\chi_{t}-P\left[\chi_{t} \mid r_{t-1}, r_{t-2}, \ldots\right]\right) r_{t-i}^{j}=0$, para $i \geq 1$, e $j=0,1,2$.

Obviamente estas definições requerem que a soma dos parâmetros do modelo $\operatorname{GARCH}(p, q)$, sem a constante, seja menor que um, isto é, $\sum_{i=1}^{p} \alpha_{i}+\sum_{i=1}^{q} \beta_{i}<1$. Também assumiremos que estes parâmetros são não negativos ${ }^{9}$, isto é, $\alpha_{i}, \beta_{j}>0$, $i=0,1, \ldots, p, j=1,2, \ldots, q$.

A definição de GARCH forte é a que foi amplamente usada por Engle (1982) e Bollerslev (1986), e geralmente com as distribuições normal ou $t$-Student. A segunda definição é devida a Weiss (1986).

Evidentemente, um GARCH forte, é também um GARCH semi-forte, e este por sua vez é também um GARCH fraco, mas esta relação não vale ao contrário.

\section{Definição 2.4.}

Sejam $r_{t}, t=1, \ldots, T$, as observações de uma série temporal, em alta frequiência, então chamaremos $r_{t}$ como variável stock, se as observações de baixa freqüência são $r_{t}$, $t=m, 2 m, \ldots, \llbracket \frac{T}{m} \rrbracket$, onde $\llbracket \bullet \rrbracket$ denota a parte inteira, e $m$ é qualquer inteiro conhecido menor que $T$.

\section{Definição 2.5.}

Sejam $r_{t}, t=1, \ldots, T$, as observações de uma série temporal, em alta frequiência, então chamaremos $r_{t}$ como variável fluxo, se as observações de baixa freqüência são $\bar{r}_{(m) t}=\sum_{i=0}^{m-1} r_{t-i}, t=m, 2 m, \ldots, \| \frac{T}{m} \rrbracket m$, onde $m$ é qualquer inteiro conhecido menor que $T$.

Note que se $y_{t}$ é uma variável stock, então $x_{t}=\Delta y_{t}=y_{t}-y_{t-1}$ é uma variável fluxo. Na prática, a série dos preços de um ativo financeiro é uma variável stock, enquanto, que os log-retornos desta série, constituem uma variável fluxo.

\footnotetext{
${ }^{9} \mathrm{Na}$ verdade, não é necessária a não negatividade dos parâmetros para ter a não negatividade de $\sigma_{t}$. Podem ser encontrados na literatura alguns exemplos construídos para mostrar isto.
} 
Drost e Nijman (1993) apresentam resultados de agregação temporal tanto para variáveis stock quanto para variáveis fluxo.

Como o nosso objetivo é analisar o comportamento dos retornos, nos centraremos no estudo do comportamento das variáveis fluxo.

\section{Proposição 2.1. (Drost e Nijman, 1993)}

Os processos GARCH(1,1) fracos simétricos, são fechados sob agregação temporal, para as variáveis fluxo.

Mais precisamente, seja $r_{t}$ um $\operatorname{GARCH}(1,1)$ fraco, com distribuição marginal simétrica, $\sigma_{t}^{2}=\alpha_{0}+\alpha r_{t-1}^{2}+\beta \sigma_{t-1}^{2}$, e curtose não condicional $\kappa_{r}=\frac{E\left[r_{t}^{4}\right]}{\left(E\left[r_{t}^{2}\right]\right)^{2}}$, então $\bar{r}_{(m) t m}$ é um $\operatorname{GARCH}(1,1)$ fraco simétrico com:

$$
\bar{\sigma}_{(m) t m}=\bar{\alpha}_{0(m)}+\bar{\alpha}_{(m)} \bar{r}_{(m) t m-m}+\bar{\beta}_{(m)} \bar{\sigma}_{(m) t m-m} .
$$

Os parâmetros do processo agregado são dados por:

$$
\begin{gathered}
\bar{\alpha}_{0(m)}=m \alpha_{0} \frac{1-(\beta+\alpha)^{m}}{1-(\beta+\alpha)} \\
\bar{\alpha}_{(m)}=(\alpha+\beta)^{m}-\bar{\beta}_{(m)} .
\end{gathered}
$$

A curtose $\bar{\kappa}_{(m) r}$ é dada por:

$$
\bar{\kappa}_{(m) r}=3+\frac{\left(\kappa_{r}-3\right)}{m}+6\left(\kappa_{r}-1\right) \frac{\left\{m-1-m(\beta+\alpha)+(\beta+\alpha)^{m}\right\}\{\alpha-\beta \alpha(\beta+\alpha)\}}{m^{2}(1-\beta-\alpha)^{2}\left(1-\beta^{2}-2 \beta \alpha\right)},
$$

e $\left|\bar{\beta}_{(m)}\right|<1$ é a solução da equação quadrática

$$
\frac{\bar{\beta}_{(m)}}{1+\bar{\beta}_{(m)}^{2}}=\frac{a\left(\beta, \alpha, \kappa_{r}, m\right)(\beta+\alpha)^{m}-b(\beta, \alpha, m)}{a\left(\beta, \alpha, \kappa_{r}, m\right)\left\{1+(\beta+\alpha)^{2 m}\right\}-2 b(\beta, \alpha, m)},
$$

com: 


$$
\begin{gathered}
a\left(\beta, \alpha, \kappa_{r}, m\right)=m(1-\beta)^{2}+2 m(m-1) \frac{(1-\beta-\alpha)^{2}\left(1-\beta^{2}-2 \beta \alpha\right)}{\left(\kappa_{r}-1\right)\left\{1-(\beta+\alpha)^{2}\right\}}+ \\
+4 \frac{\left\{m-1-m(\beta+\alpha)+(\beta+\alpha)^{m}\right\}\{\alpha-\beta \alpha(\beta+\alpha)\}}{1-(\beta+\alpha)^{2}} \\
b(\beta, \alpha, m)=\{\alpha-\beta \alpha(\beta+\alpha)\} \frac{1-(\beta+\alpha)^{2 m}}{1-(\beta+\alpha)^{2}} .
\end{gathered}
$$

Pode-se mostrar facilmente que para os modelos GARCH fortes, o coeficiente de curtose da série, relaciona-se com o coeficiente de curtose das inovações como:

$$
\kappa_{r}=\kappa_{\varepsilon} \frac{1-(\beta+\alpha)^{2}}{1-(\beta+\alpha)^{2}-\left(\kappa_{\varepsilon}-1\right) \alpha^{2}}
$$

De fato, temos que:

$$
E\left(r_{t}^{2}\right)=E\left(\sigma_{t}^{2} \varepsilon_{t}^{2}\right)=E\left(\sigma_{t}^{2}\right)
$$

E usando esta relação,

$$
\begin{aligned}
E\left(r_{t}^{2}\right) & =E\left(\alpha_{0}+\alpha r_{t-1}^{2}+\beta \sigma_{t-1}^{2}\right) \\
& =\alpha_{0}+\alpha E\left(r_{t-1}^{2}\right)+\beta E\left(\sigma_{t-1}^{2}\right) \\
& =\alpha_{0}+(\alpha+\beta) E\left(r_{t-1}^{2}\right) .
\end{aligned}
$$

E pela estacionariedade dos processos GARCH fortes tem-se

$$
E\left(r_{t}^{2}\right)=\frac{\alpha_{0}}{1-(\alpha+\beta)}
$$

Por outro lado

$$
E\left(r_{t}^{4}\right)=E\left(\sigma_{t}^{4} \varepsilon_{t}^{4}\right)=E\left(\sigma_{t}^{4}\right) E\left(\varepsilon_{t}^{4}\right)
$$

e 


$$
\begin{aligned}
E\left(\sigma_{t}^{4}\right) & =\alpha_{0}^{2}+\alpha^{2} E\left(r_{t-1}^{4}\right)+\beta^{2} E\left(\sigma_{t-1}^{4}\right)+2 \alpha_{0} \alpha E\left(r_{t-1}^{2}\right)+ \\
& +2 \alpha_{0} \beta E\left(\sigma_{t-1}^{2}\right)+2 \alpha \beta E\left(r_{t-1}^{2} \sigma_{t-1}^{2}\right)
\end{aligned}
$$

logo, usando (2.2) temos,

$$
\left[1-\left(\alpha^{2} E\left(\varepsilon_{t-1}^{4}\right)+\beta^{2}+2 \alpha \beta\right)\right] E\left(\sigma_{t}^{4}\right)=\alpha_{0}^{2}+2 \alpha_{0}(\alpha+\beta) E\left(r_{t-1}^{2}\right)
$$

o que nos dá

$$
E\left(r_{t}^{4}\right)=\frac{\alpha_{0}^{2}+2 \alpha_{0}(\alpha+\beta) E\left(r_{t-1}^{2}\right)}{\left[1-\left(\alpha^{2} E\left(\varepsilon_{t-1}^{4}\right)+\beta^{2}+2 \alpha \beta\right)\right]} E\left(\varepsilon_{t}^{4}\right)
$$

Juntando, agora, (2.3) e (2.6) temos:

$$
\begin{aligned}
\frac{E\left(r_{t}^{4}\right)}{\left\{E\left(r_{t}^{2}\right)\right\}^{2}} & =\frac{\alpha_{0}(1+(\alpha+\beta)) E\left(r_{t-1}^{2}\right)}{\left[1-\left(\alpha^{2} E\left(\varepsilon_{t-1}^{4}\right)+\beta^{2}+2 \alpha \beta\right)\right]\left[E\left(r_{t-1}^{2}\right)\right]^{2}} E\left(\varepsilon_{t}^{4}\right) \\
& =\frac{\left(1-(\alpha+\beta)^{2}\right)}{\left[1-\left(\alpha^{2} E\left(\varepsilon_{t-1}^{4}\right)+\beta^{2}+2 \alpha \beta\right)\right]} E\left(\varepsilon_{t}^{4}\right)
\end{aligned}
$$

de donde obtemos

$$
\frac{E\left(r_{t}^{4}\right)}{\left\{E\left(r_{t}^{2}\right)\right\}^{2}}=\frac{\left(1-(\alpha+\beta)^{2}\right)}{\left[1-(\alpha+\beta)^{2}-\alpha^{2}\left(E\left(\varepsilon_{t-1}^{4}\right)-1\right)\right]} E\left(\varepsilon_{t}^{4}\right)
$$

e portanto a relação (2.1) vale.

A principal diferença entre as variáveis fluxo e stock, no que se refere a agregação temporal, é que nas variáveis fluxo os parâmetros da equação de variância do modelo de baixa freqüência agregado dependem não somente dos parâmetros de variância do modelo de alta frequiência, mas também da curtose das observações; enquanto que nas variáveis stock os parâmetros da equação de variância do modelo de baixa freqüência agregado dependem dos parâmetros de variância do modelo de alta freqüência, e não da curtose. 
Note-se que a heteroscedasticidade condicional desaparece quando a agregação é grande $\left(\bar{\beta}_{(m)}, \bar{\alpha}_{(m)} \rightarrow 0, m \rightarrow \infty\right)$, tal como foi mostrado por Diebold (1988). Também pode ser visto que a curtose converge para 3 quando $m \rightarrow \infty$, sugerindo normalidade assintótica de $\frac{\bar{r}_{(m) t}}{\sqrt{m}}$ e que ademais os processos de freqüência muita alta convergem a um I-GARCH ( $\beta \rightarrow 1, \alpha \rightarrow 0, m \rightarrow 0$ ).

A definição de GARCH fraco é bastante geral e como foi visto engloba os processos GARCH semi-fortes, que por sua vez, englobam os processos GARCH fortes.

Como existe uma correspondência bi-unívoca entre os parâmetros de baixa e alta freqüência, podemos obter estimadores consistentes dos parâmetros de alta freqüência a partir dos parâmetros de baixa freqüência ou vice-versa, assim se assumimos que um processo GARCH explica o comportamento dos dados em uma freqüência, podemos determinar os parâmetros consistentes do processo GARCH em uma outra freqüência, por meio da agregação ou desagregação temporal. Mas deve-se ter cuidado sobre o processo GARCH agregado ou desagregado, pois se soubermos que ele é fraco, não necessariamente é forte ou semi-forte, isto é, somente podemos afirmar que os processos GARCH fracos são fechados sob agregação temporal.

Como exemplo da proposição anterior podemos ver, por exemplo, que os processos $\mathrm{ARCH}(1)$ não são fechados sob agregação temporal, para as variáveis fluxo, pois modelos ARCH(1) em alta frequiência quando agregados, geram modelos $\operatorname{GARCH}(1,1)$ de baixa freqüência, com o parâmetro da parte GARCH não necessariamente igual a zero.

$\mathrm{Na}$ Ilustração 1 realizamos a simulação da agregação temporal de um processo GARCH $(1,1)$ para variáveis fluxo. Os parâmetros inicias para a agregação temporal são $(0,0.03,0.85,3.1),(0,0.03,0.85,9.5),(0,0.05,0.8,3.1)$ e $(0,0.05,0.8,9.5)$, que correspondem aos valores iniciais de $\left(\alpha_{0}, \alpha, \beta, \kappa_{r}\right)$, onde $\kappa_{r}$ é o coeficiente de curtose inicial da série.

Como pode-se observar, unicamente os parâmetros da equação de variância não determinam o comportamento sob agregação temporal sobre variáveis fluxo, isto é, precisamos do coeficiente de curtose, além dos parâmetros da equação de variância, ou seja, a agregação temporal é diferente, quando consideramos diferentes coeficientes de curtoses, embora os parâmetros da equação de variância sejam os mesmos, tal como podemos ver na Ilustração 1.

Drost e Nijman (1992) mostram que é melhor considerar a curtose, em alta frequiência, como uma função dos parâmetros da equação de variância de baixa freqüência, em lugar de considerá-la como um parâmetro independente, quando é assumida a hipótese que os dados são gerados por um modelo GARCH em uma determinada alta freqüência.

$\mathrm{Na}$ Tabela 1 podem-se constatar alguns dos resultados anteriormente mencionados, como a heteroscedasticidade condicional desaparece quando a agregação é grande, isto é, $\left(\bar{\beta}_{(m)}, \bar{\alpha}_{(m)} \rightarrow 0, m \rightarrow \infty\right)$. 


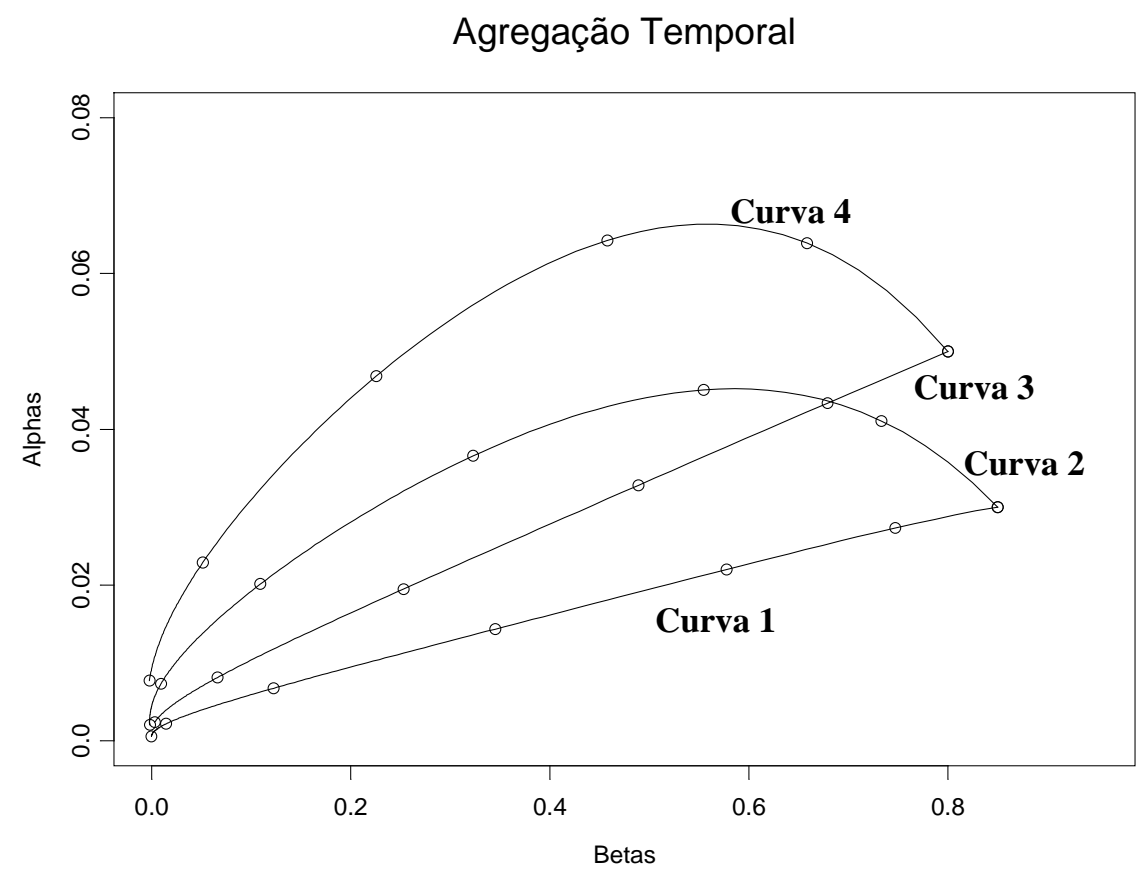

Ilustração 1.- Agregação temporal de um $\operatorname{GARCH}(1,1)$ para diferentes níveis de agregação, para variáveis fluxo.

\begin{tabular}{|c|c|c|c|c|c|c|}
\hline \multirow{2}{*}{ Nivel } & \multicolumn{3}{|c|}{ Curva 1} & \multicolumn{3}{|c|}{ Curva 2} \\
\hline & Alpha & Beta & Curtose & Alpha & Beta & Curtose \\
\hline 1 & 0.030 & 0.85 & 3.10 & 0.030 & 0.850 & 9.50 \\
\hline 2 & 0.027 & 0.75 & 3.16 & 0.041 & 0.733 & 6.68 \\
\hline 4 & 0.022 & 0.58 & 3.17 & 0.045 & 0.555 & 5.21 \\
\hline 8 & 0.014 & 0.35 & 3.16 & 0.037 & 0.323 & 4.40 \\
\hline 16 & 0.007 & 0.12 & 3.13 & 0.020 & 0.109 & 3.89 \\
\hline 32 & 0.002 & 0.01 & 3.08 & 0.007 & 0.009 & 3.53 \\
\hline \multirow{2}{*}{ Nivel } & \multicolumn{3}{|c|}{ Curva 3} & \multicolumn{3}{|c|}{ Curva 4} \\
\hline & Alpha & Beta & Curtose & Alpha & Beta & Curtose \\
\hline 1 & 0.050 & 0.80 & 3.10 & 0.050 & 0.800 & 9.50 \\
\hline 2 & 0.043 & 0.68 & 3.23 & 0.064 & 0.659 & 6.98 \\
\hline 4 & 0.033 & 0.49 & 3.27 & 0.064 & 0.458 & 5.61 \\
\hline 8 & 0.020 & 0.25 & 3.25 & 0.047 & 0.226 & 4.77 \\
\hline 16 & 0.008 & 0.07 & 3.19 & 0.023 & 0.051 & 4.15 \\
\hline 32 & 0.002 & 0.00 & 3.12 & 0.008 & 0.000 & 3.68 \\
\hline
\end{tabular}

Tabela 1.- Valores da agregação temporal de um processo $\operatorname{GARCH}(1,1)$ para diferentes níveis de agregação, para variáveis fluxo. 
Também pode ser visto que a curtose converge para 3 quando $m \rightarrow \infty$ sugerindo normalidade asintótica de $\frac{\bar{r}_{(m) t}}{\sqrt{m}}$ e que ademais os processos de freqüência muita alta convergem a um I-GARCH ( $\beta \rightarrow 1, \alpha \rightarrow 0, m \rightarrow 0)$.

Um resultado interessante também que foi mostrado por Drost e Nijman (1993) é que tanto os modelos GARCH fortes, quanto os GARCH semi-fortes, não são fechados sob agregação temporal, e que a classe de modelos ARMA com erros GARCH fracos simétricos é fechada sob agregação temporal, tanto para as variáveis fluxo quanto para as variáveis stock. Ainda mais, eles mostraram que se uma séria é explicada por um processo $\operatorname{GARCH}(p, q)$ fraco simétrico, então a agregação temporal é explicada por um processo $\operatorname{GARCH}(r, r)$ fraco simétrico, onde $r=\max (p, q)$.

A Tabela 2 mostra alguns exemplos de agregação temporal para modelos de baixa frequiência, para variáveis fluxo. Assim, por exemplo, um modelo $\operatorname{ARCH}(q)$ de alta freqüência, quando agregado temporalmente, pertence à família de modelos $\operatorname{GARCH}(q, q)$, se a agregação temporal é considerando-se variáveis fluxo.

Na seção 2.5.2.1 vamos realizar o estudo da agregação temporal para o índice da Bolsa de Valores do Estado de São Paulo, Ibovespa. Como este ativo, do mesmo modo que a maior parte das "equities", fecha nos horários não comerciais, isto pode afetar os resultados da agregação temporal, embora isto seria minimizado se o ponto de partida fosse uma série diária.

No entanto, realizaremos a agregação temporal para um tipo de ativo financeiro que é muito estudado, e que tem a atrativa característica de ser negociado 24 horas por dia sete dias por semana, a saber, as "currencies". Embora isto seja certo para muitas moedas, isto é, elas podem ser negociadas a qualquer horário e em qualquer dia, para outras moedas isto não acontece com facilidade, pelo que aparecem grandes oscilações na série, principalmente nos horários de abertura dos mercados financeiros. Por este motivo é interessante escolher uma moeda líquida. Mesmo tendo uma moeda líquida a negociação em certos horários poderia resultar difícil. O Euro é o ativo financeiro mais líquido do mundo.

\begin{tabular}{|ll|}
\hline Modelo de Alta Freqüência & Modelo de Baixa Freqüência \\
\hline \hline $\operatorname{ARCH}(q)$ & $\operatorname{GARCH}(q, q)$ \\
$\operatorname{GARCH}(1, q)$ & $\operatorname{GARCH}(q, q)$ \\
$\operatorname{MA}(1)+\operatorname{ARCH}(q)$ & $\operatorname{MA}(1)+\operatorname{GARCH}(q+1, q+1)$ \\
$\operatorname{AR}(1)+\operatorname{ARCH}(q)$ & $\operatorname{ARMA}(1,1)+\operatorname{GARCH}(q+1, q+1)$ \\
$\operatorname{ARMA}(1,1)+\operatorname{GARCH}(1, q+1)$ & $\operatorname{ARMA}(1,1)+\operatorname{GARCH}(q+1, q+1)$ \\
\hline
\end{tabular}

Tabela 2.- Limites superiores das ordens dos modelos de baixa frequiência que são gerados a partir da agregação temporal de modelos de alta freqüência, para variáveis fluxo. 


\subsection{Aplicações da Agregação Temporal.}

Parece então natural realizar um teste e comprovar se um ajuste de dados em alta freqüência mediante processos GARCH faz sentido.

A dúvida que existe é sobre as estimações dos parâmetros já que esta pode não ser consistente se consideramos um processo GARCH forte como o gerador dos dados.

Como nosso objetivo é a modelagem de dados de alta frequiência, mais de que na agregação temporal, estamos interessados na desagregação temporal, isto é, ver se os modelos que explicam o comportamento dos dados em baixa freqüência servem também em alta frequiência.

Muitos documentos já mostraram a eficácia de um ajuste GARCH para séries financeiras diárias, por isso, este é mais um motivo de tomar como ponto de partida uma série de frequiência diária e fazer agregação e desagregação temporal.

Existem vários artigos que apontam que o processo $\operatorname{GARCH}(1,1)$ especifica corretamente, tanto a série do Euro quanto a do índice Ibovespa.

Antes de começar com a modelagem, vamos mencionar os principais fatos estilizados das séries financeiras e ver quais destes fatos estão presentes nestas duas séries e assim entender melhor porque muitos artigos apontam que o processo $\operatorname{GARCH}(1,1)$ especifica corretamente estas duas séries financeiras.

\subsubsection{Principais fatos estilizados das séries financeiras.}

Variáveis financeiras, tal como os preços de um ativo, são afetados pela chegada de novas informações ao mercado financeiro. Dias em que novidades não surgem, sejam "boas" ou "ruins", são caracterizados por pouca movimentação ou alteração dos preços, uma vez que a qualidade da informação não está em pauta. Entretanto, dias carregados de novas informações têm efeito justamente oposto, ou seja, apresentam alta variação nos preços. Tal constatação conduziu Mandelbrot e Taylor (1967) e Clark (1973) a modelarem preços de ações como processos estocásticos subordinados, ou seja, ao invés de modelarem os preços como função do tempo calendário, quer diário, semanal, mensal, etc, eles modelaram como função da chegada de nova informação ao mercado, a qual é um processo que flui aleatoriamente ao longo do tempo. Assim, segundo esta abordagem, não olhamos mais para nosso processo de interesse evoluindo ao longo do tempo calendário que é igualmente espaçado, mas sim evoluindo de acordo com a chegada de nova informação, originando uma nova dimensão temporal, a qual chamaremos de tempo operacional. Desta maneira, fica caracterizado o que denominamos de Deformação Temporal. Como não é de nosso interesse aprofundar sob este tema, tomaremos como tempo operacional aquele que é conhecido como tempo de negócios, "business time", e que simplesmente descarta do tempo físico, aquelas horas ou períodos onde o mercado é fechado ou praticamente não existem transações.

Em muitas ocasiões, modelar o preço de um ativo financeiro para entender o comportamento do mercado, resume-se a modelar a volatilidade atrelada a este ativo. 
Este se deve principalmente à natureza e comportamento dos preços dos ativos financeiros, onde predizer o preço de um ativo resulta praticamente impossível e irreal. A volatilidade nos indica se o preço de um ativo está variando pouco ou muito, ou seja, ela é uma medida da incerteza quanto às variações de preço. Épocas em que a variabilidade dos preços está muito alta são aquelas que possibilitam os maiores lucros ou, também as maiores perdas; isto é, são aquelas em que o risco é maior. Já quando a volatilidade é baixa, o risco é menor. A relação é óbvia e, naturalmente, um investidor somente assumiria uma posição de alto risco caso houvesse a possibilidade de um grande retorno. Em outras palavras existe uma relação direta entre risco e retorno.

Dados financeiros apresentam certas características peculiares, fatos estilizados, como os mostrados por Taylor (1986). Este é o caso dos log-retornos, que chamaremos a partir de agora simplesmente de retornos de ativos financeiros, que são definidos por $y_{t}=\log x_{t}-\log x_{t-1}{ }^{10}$ onde $x_{t}$ é o preço do ativo no instante $t$. Os principais fatos estilizados (características) encontrados na literatura são os seguintes:

i) Os retornos são, em geral, não autocorrelacionados ou de autocorrelação desprezível;

ii) Os quadrados dos retornos são autocorrelacionados, com uma pequena autocorrelação de primeira ordem e uma subsequente queda bastante lenta;

iii) Existem agrupamentos com diferentes níveis de volatilidade;

iv) A distribuição não condicional dos retornos possui caudas pesadas em relação à distribuição normal;

v) Para alguns tipos de retornos, a volatilidade reage de maneira diferenciada se os preços estão aumentando ou declinando. Sabe-se que as subidas são lentas e a quedas são abruptas indicando que neste último caso deveríamos ter uma maior volatilidade.

Note que estas características guardam algumas relações entre si, dando origem a conclusões semelhantes. Por exemplo, se os retornos tivessem distribuição normal, a característica (i) inviabilizaria a (ii), pois a inexistência de correlação para variáveis conjuntamente gaussianas implica independência de quaisquer funções disjuntas dessas variáveis. Do mesmo modo, a característica (iv) inviabiliza a distribuição normal. De fato, a autocorrelação dos quadrados dos retornos tem forte relação com distribuições com caudas pesadas, pois, quando surgem valores extremos, quer positivos ou negativos, devido à autocorrelação, haverá tendência de que os próximos quadrados também sejam extremos, originando um agrupamento de valores extremos e portanto caudas pesadas. De forma análoga, as características (ii) e (iii) estão intimamente relacionadas. Quando os quadrados dos retornos são autocorrelacionados, é de se esperar que valores grandes, em módulo, serão seguidos por valores também grandes,

${ }^{10} \mathrm{O}$ uso de log-retornos é principalmente devido ao fato de que os preços não são séries estacionárias. O logaritmo ajuda a reduzir a variância e a diferença ajuda a tirar a tendência das séries. 
enquanto que valores pequenos estarão próximos de valores pequenos, causando assim os agrupamentos de volatilidade.

Modelos lineares gaussianos de séries temporais são incapazes de incorporar alguns dos fatos estilizados apresentados acima. Assim, a partir da década passada, foram desenvolvidos modelos estatísticos que eram capazes de incorporar estes fatos estilizados. Os principais tipos de modelos que são capazes de incorporar estes fatos são os ARCH ("Autoregressive Condicional Heteroskedasticity"), GARCH (“Generalized ARCH”) e de Volatilidade Estocástica (V.E.).

\subsubsection{Fatos Estilizados na série do Ibovespa.}

Dispomos de uma série de dados do índice Ibovespa que vai desde o 2 de janeiro de 1998 ás 11 h00 até o 13 de agosto do 2003 ás 17 h00 em intervalos de 15 minutos.

O primeiro passo é obter destes dados uma série para poder trabalhar, isto é, filtrar e tratar a série.

A hora de abertura e fechamento da bolsa varia durante o ano, portanto os horários de recompilação de dados do índice, variam também dia a dia. Assim, por exemplo, temos dados do índice às $10 \mathrm{~h} 00$ ou às $19 \mathrm{~h} 00$.

No entanto, em alguns horários a bolsa funciona de maneira diferente, à maneira de leilão, que serve para puxar o valor do índice.

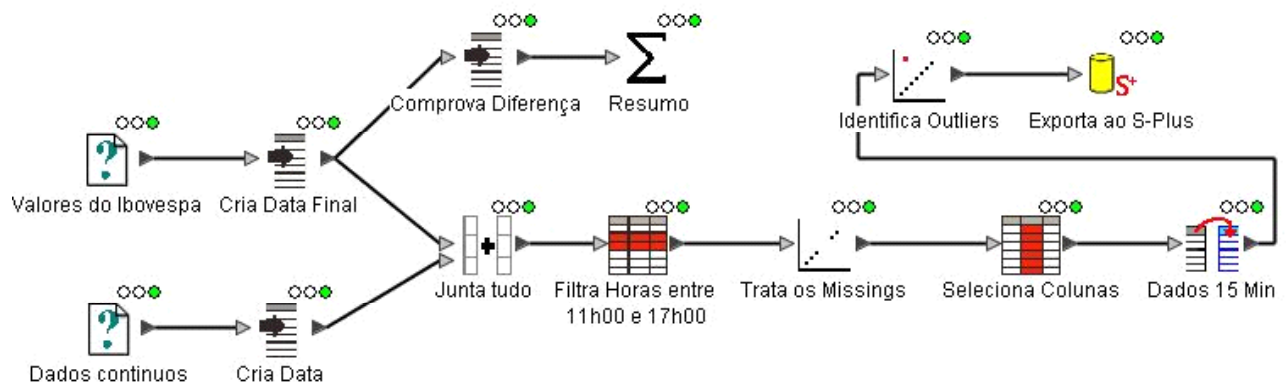

Ilustração 2.- Obtenção dos dados do índice Ibovespa.

Então o primeiro passo é uniformizar os horários dos valores coletados, seja via interpolação dos dados faltantes ou eliminando os dados onde a informação é incompleta.

$\mathrm{Na}$ realidade, isto não afetará nossa análise, pois partiremos de uma série em alta freqüência assim tratada, à que chamaremos do índice Ibovespa. Sob esta série realizaremos agregações temporais para partir de uma série diária no estudo seguinte.

$\mathrm{Na}$ Ilustração 2 mostramos o procedimento realizado no Insigthful Miner para a obtenção e filtragem dos dados. 
Selecionamos os valores do índice Ibovespa desde as $11 \mathrm{~h} 00$ até as $17 \mathrm{~h} 00$ em intervalos de 15 minutos, de 2 de janeiro de 1998 até o 13 de agosto do 2003, resultando num total de 34450 observações, durante 1378 dias.

Mesmo assim, existiram 125 valores perdidos por falha na captura dos dados ou porque a bolsa parou em alguns horários. Isto pode ter acontecido durante os jogos da seleção brasileira no mundial de futebol, por exemplo.

Como durante estes horários não existiu negociação o valor "real" do índice é o último valor negociado, é assim que tratamos os valores faltantes, isto é copiando a última observação no lugar da observação faltante.

Agora temos que tratar os dados extraídos de possíveis outliers. Para isso primeiro vamos identificar os possíveis outliers e tentar corrigi-los ou então substituí-los pela interpolação linear dos valores anterior e posterior.
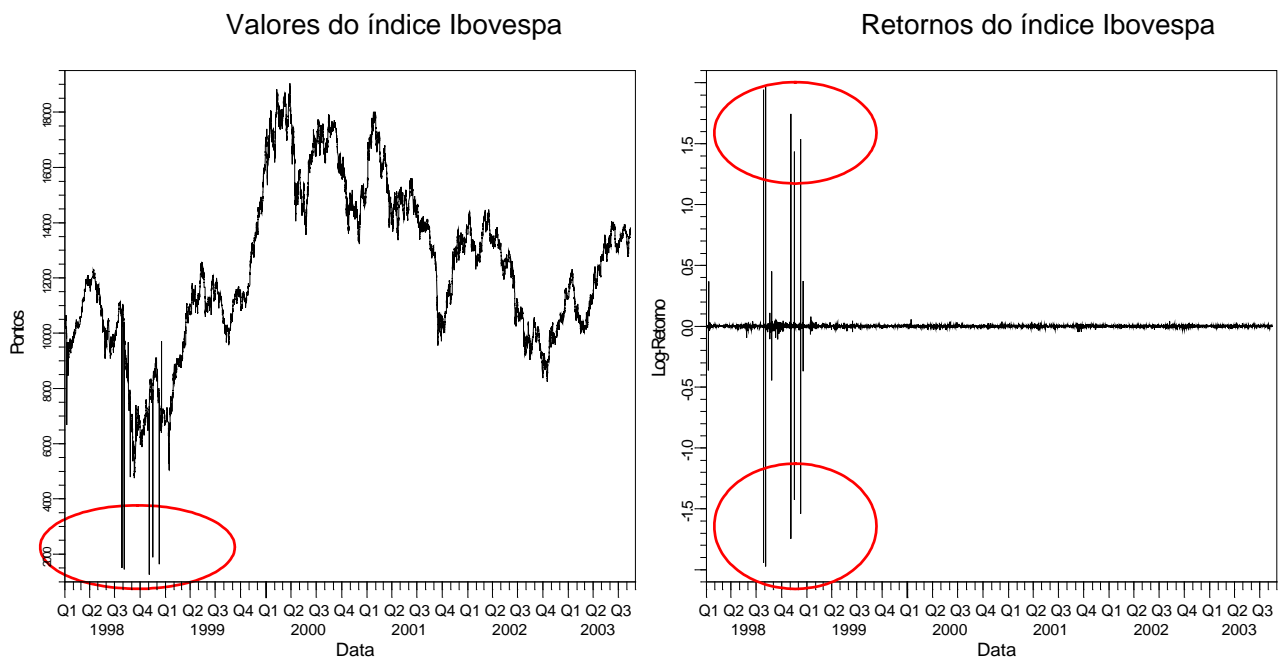

Ilustração 3.- Valores e retornos do índice Ibovespa a intervalos de 15 minutos sem tratamento de outliers.

Na Ilustração 3 podemos identificar claramente alguns outliers que podem vir de erros na extração ou manipulação dos dados. Como se pode observar, o gráfico dos retornos ajuda a identificar mais claramente os outliers.

Então o primeiro passo é limpar estas séries dos outliers. A Ilustração 4 mostra o gráfico da série e dos retornos do índice Ibovespa com freqüência de 15 minutos, depois de que os outliers foram corrigidos (substituí-los pela interpolação linear dos valores anterior e posterior).

Uma vez corrigidos e filtrados os dados procedemos a obter o log-retorno do índice Ibovespa. A variável assim definida é uma variável fluxo. Com estes retornos realizaremos as agregações temporais.

Vamos fazer 4 agregações para variáveis fluxo, a primeira agregação, de 5 observações, corresponde a dados a cada 1,25 horas, a segunda agregação, de 25 
observações, corresponde a dados diários, a terceira agregação, de 125 observações, corresponde a dados semanais e última agregação, de 500 observações, corresponde a dados mensais.
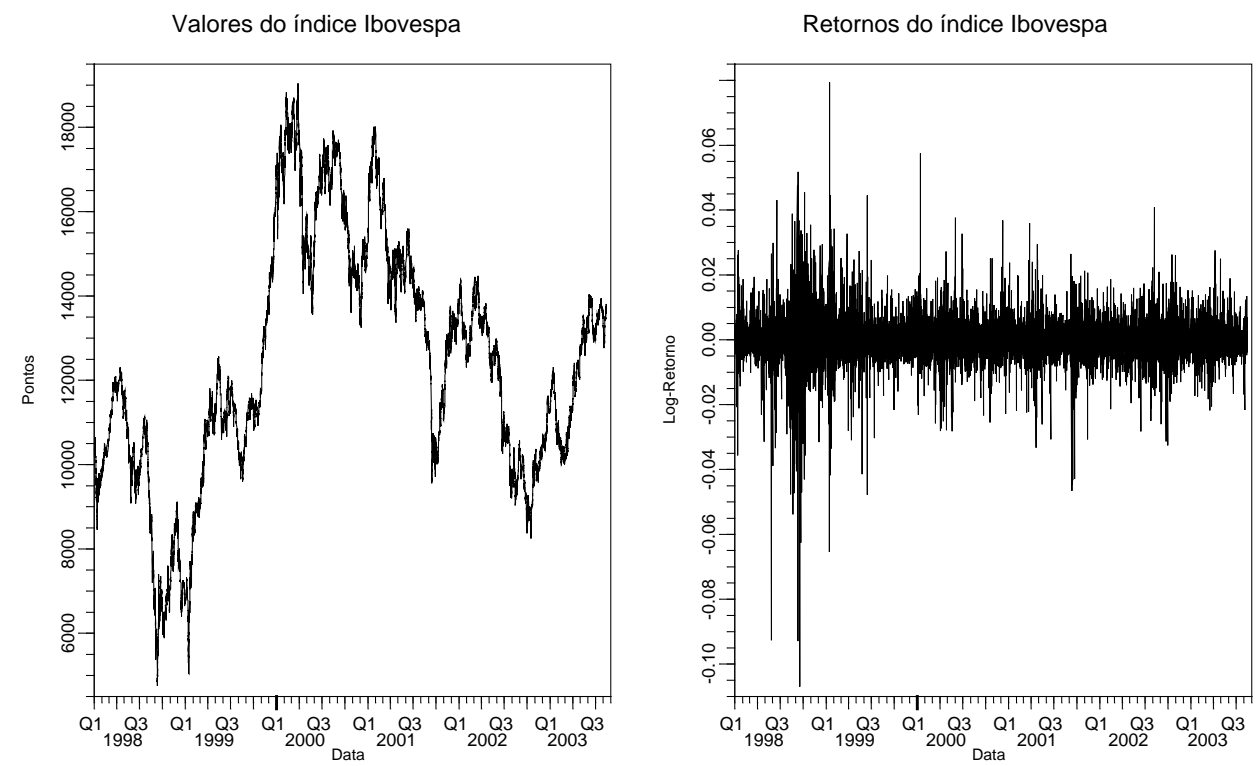

Ilustração 4.- Valores e retornos do índice Ibovespa a intervalos de 15 minutos depois do tratamento de outliers.
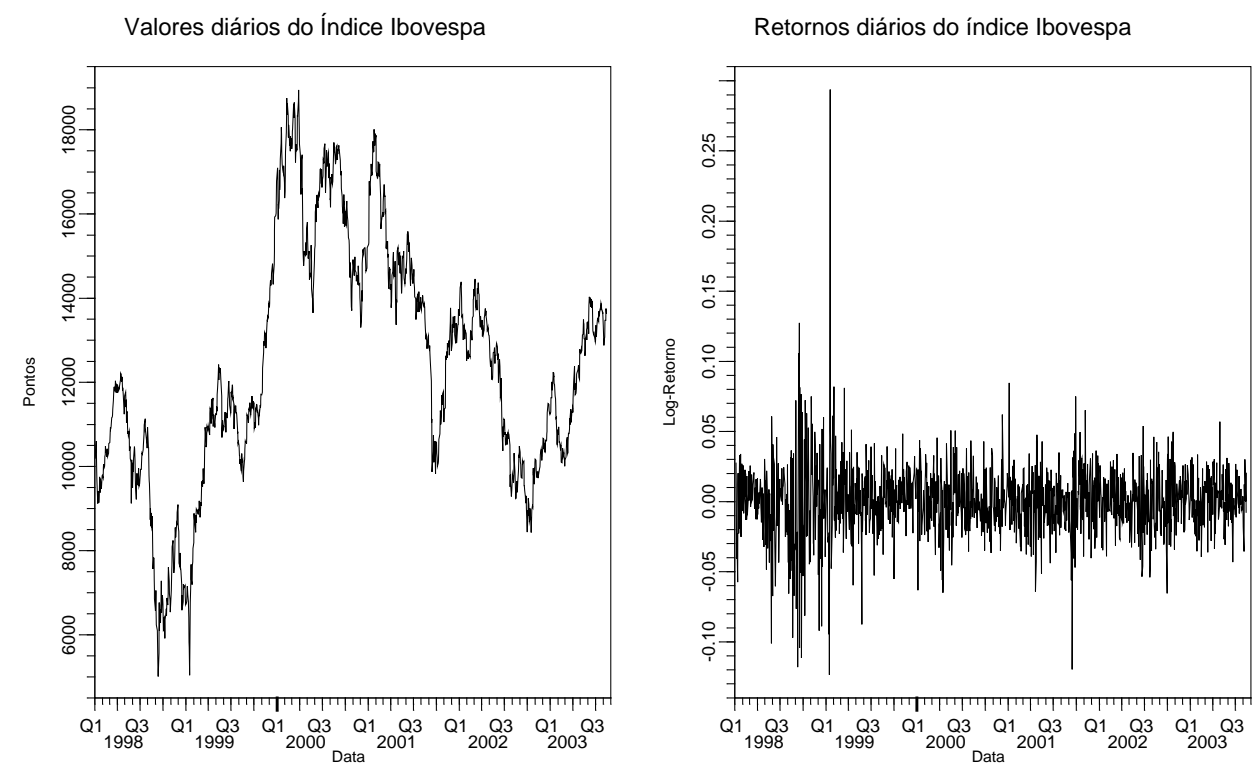

Ilustração 5.- Valores e retornos diários do índice Ibovespa. 
Como tínhamos mencionado anteriormente usaremos a série diária como ponto de partida para fazer o estudo de agregação e desagregação temporal.

Então, primeiramente obtemos os retornos da série com frequiência de 15 minutos, e como em cada dia temos 25 observações (retornos das 11 h00 até as 17 h00 incluídas, em intervalos de 15 minutos), fazemos a agregação temporal das 25 observações considerando a definição de variáveis fluxo.

$\mathrm{Na}$ Ilustração 5 podem-se ver os valores diários do índice Ibovespa. Como pode-se notar, embora já tenha havido um tratamento de outliers, ainda aparecem certos valores extremos que são consistentes com series de retornos em alta freqüência, tal como mencionamos nos fatos estilizados.

Observando a série dos retornos, também podem se ver certos agrupamentos destes, em termos de volatilidade, embora seja difícil identificar quantos e onde começam e terminam.

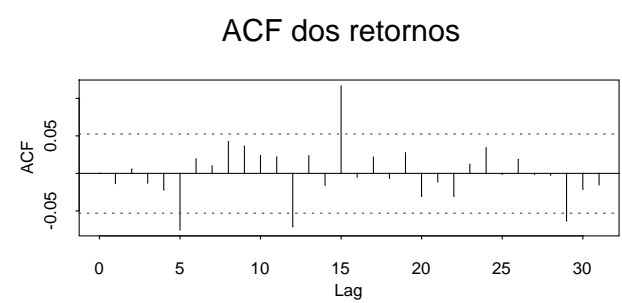

ACF dos retornos quadrados

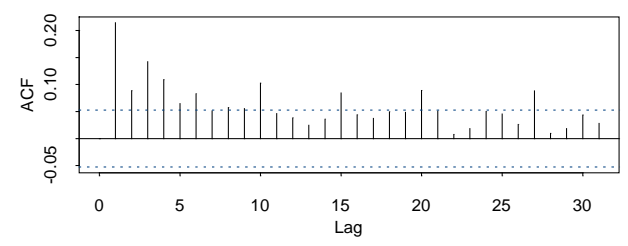

Retornos quadrados

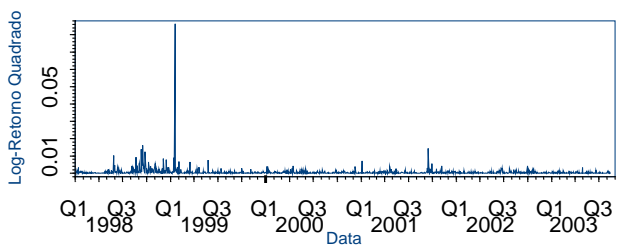

PACF dos retornos

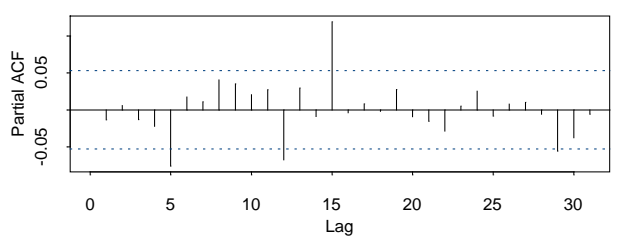

PACF dos retornos quadrados

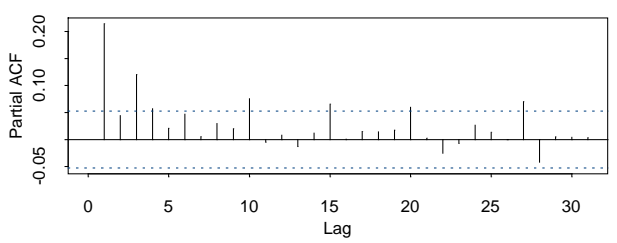

Histograma dos Retornos Quadrados

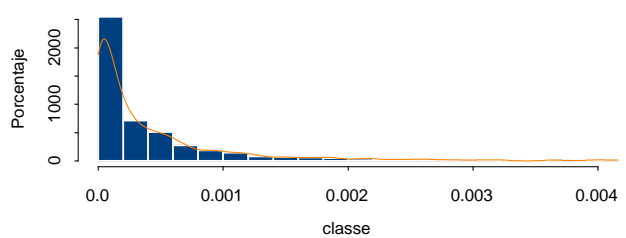

Ilustração 6.- Função de autocorrelação e autocorrelação parcial dos retornos e dos quadrados dos retornos do índice Ibovespa, plot e histograma dos quadrados dos retornos, freqüência diária.

Obviamente, esta distinção entre os agrupamentos não é muito perceptível e, pode ser melhor entendida quando observarmos os gráficos das funções de autocorrelação dos retornos e de seus quadrados, dado pela Ilustração 6. Nesta figura, vemos claramente 
que as autocorrelações de ordem 5, 12, 15 e 29 dos retornos são significantes, rejeitando-se a hipótese de ruído branco e, portanto, violando o primeiro dos fatos estilizados. Podemos notar também que a primeira autocorrelação dos quadrados dos retornos é significante e após esta ordem a função de autocorrelação decai lentamente, confirmando o segundo fato estilizado.

A Ilustração 7 apresenta o histograma dos retornos do índice Ibovespa e a estimação não paramétrica da densidade, com largura de faixa calculada segundo o método de validação cruzada não viciada.

Como tínhamos mencionado anteriormente, a presença de muitos eventos extremos faz com que o histograma apresente caudas pesadas, o qual parece validar os fatos estilizados, mas poderíamos testar a normalidade dos retornos para poder confirmar efetivamente o fato estilizado que os retornos apresentam caudas pesadas em relação à distribuição normal.

Outro assunto interessante é que, embora exista uma aparente simetria visual nos retornos, este não é um fato comum, especialmente num ativo de equity, pois tende a existir um maior número de retornos positivos que negativos, o qual faz que o histograma seja assimétrico à direita. Isto ocorre pela natureza que tem as bolsas de ter tendência crescente. De fato, quando realizamos o teste de hipótese, esta assimetria é confirmada, o qual não é facilmente visível na Ilustração 7.

O coeficiente de assimetria dos retornos é 0.75 e o coeficiente de curtose é 13.96 , e ao se testar hipóteses a respeito da assimetria e de curtose, se rejeitam as hipóteses de não existência de assimetria e excesso de curtose em relação à distribuição normal, a um nível descritivo inferior a $0,01 \%$.

Histograma dos Log-Retornos diários

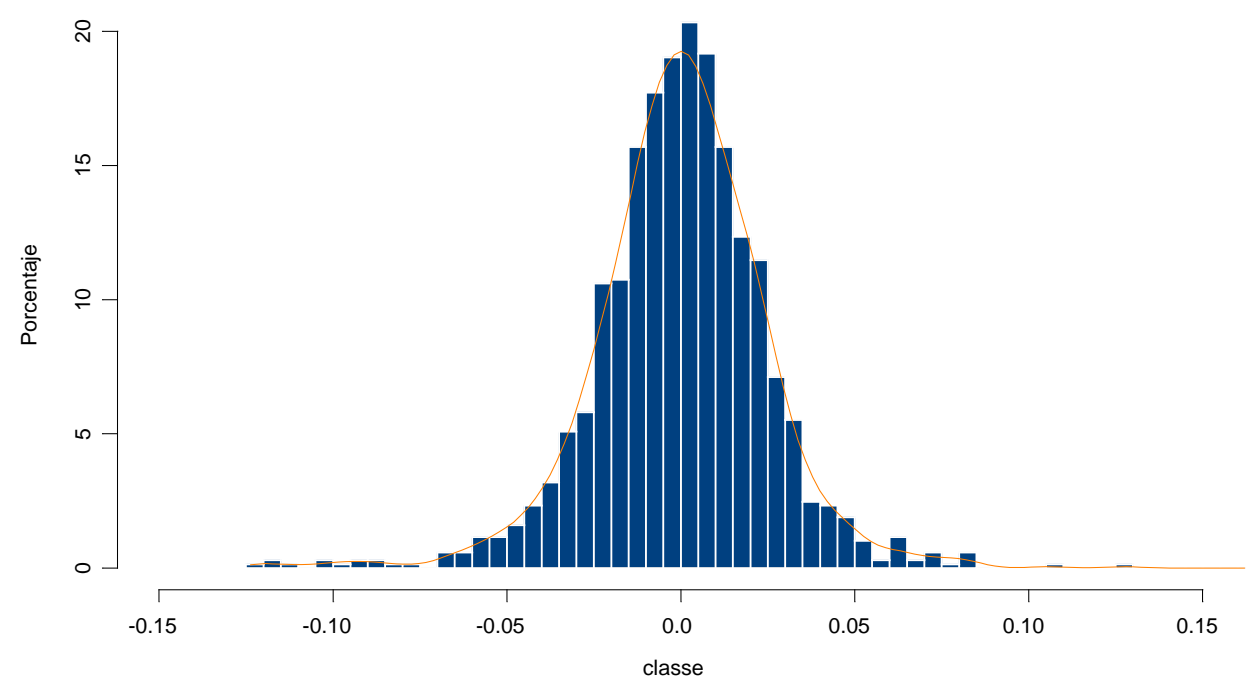

Ilustração 7.- Histograma dos retornos do índice Ibovespa, frequiência diária. 
O código com as análises descritivas para a série do Ibovespa foram feitos no S-Plus (o tratamento foi feito no Insightful Miner).

É importante mencionar que a agregação temporal deve ser realizada segundo a definição de variáveis fluxo (para os retornos). $O$ fato de não considerar esta definição pode não validar as formulas de agregação temporal. Isto pode ocorrer, por exemplo, se em lugar de agregar 125 retornos para obter os retornos diários, tomamos o último valor negociado no dia e calculamos os retornos.

\subsubsection{Fatos Estilizados na série do Euro-Dólar.}

Dispomos de uma série de dados, já tratada e filtrada, da taxa de câmbio Euro-Dólar que vai desde o 1 de janeiro de 1999 ás $02 \mathrm{~h} 48 \mathrm{~m} 35$ s até o 31 de dezembro do 2002 ás $23 \mathrm{~h} 32 \mathrm{~m} 38$ s tick by tick. Dispõe-se então de 20.989.044 ticks com os preços Bid e Ask e a fonte da informação.

O primeiro passo é obter uma série com o preço médio a intervalos de 5 minutos, last price.
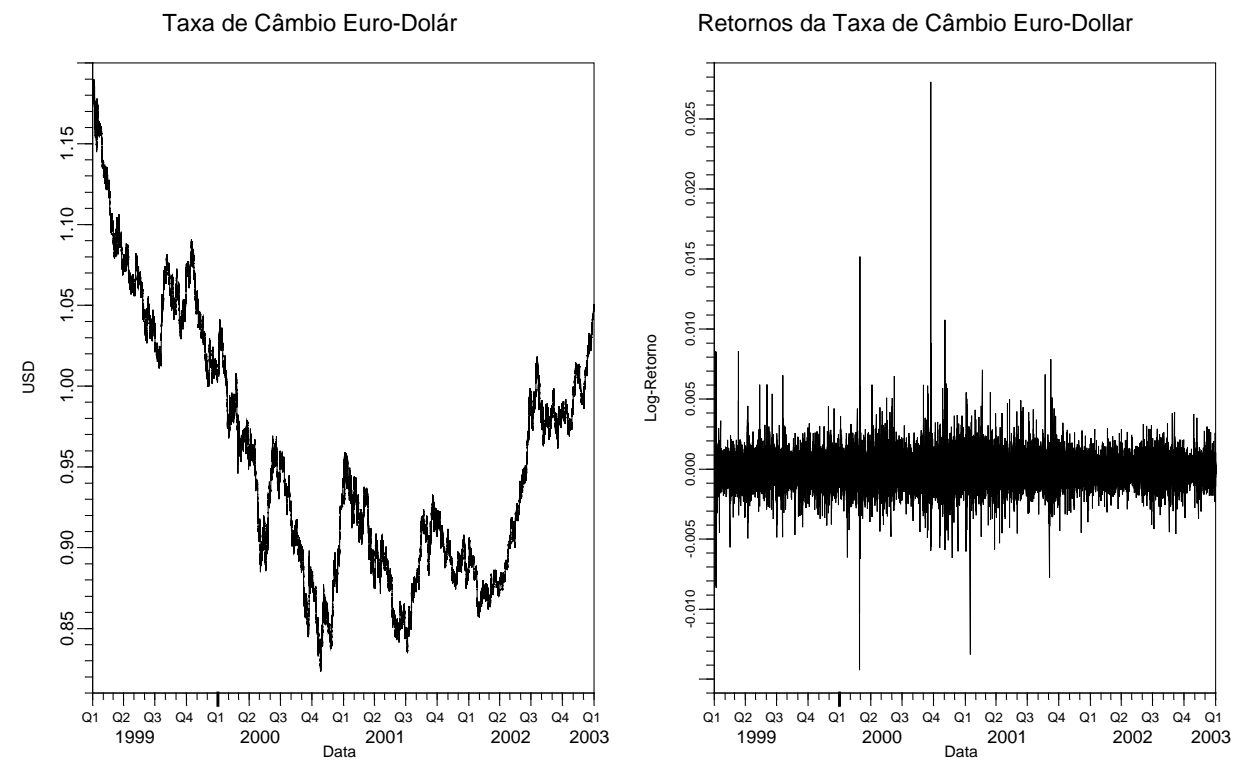

Ilustração 8.- Valores e retornos da taxa de câmbio Euro-Dólar a intervalos de 5 minutos, filtrada.

O mercado de FX é contínuo, no sentido que se realizam transações em qualquer dia do ano e em qualquer horário, mais ainda para o Euro-Dólar que é um dos ativos mais líquidos do mundo. No entanto, os sábados e domingos e feriados (dos Estados Unidos) são dias de pouca atividade e movimentação do mercado. Então filtraremos nossa série destes valores. 
Como na série do Ibovespa, isto não afetara nossa análise, pois partiremos de uma série em alta frequiência assim tratada, que chamaremos do taxa de câmbio Euro-Dólar e para esta série realizaremos agregações temporais, obtendo-se uma série de menor frequiência no estudo seguinte.

A série a intervalos de 5 minutos reduz-se consideravelmente de 20.989 .044 a 288860 observações depois de $\operatorname{alisar}^{11}$ a série e filtrar a dias úteis.

Como tínhamos mencionado antes, esta é uma série que já foi filtrada de possíveis outliers, isto também podemos observar na Ilustração 8, onde estão os valores e os retornos da taxa de câmbio Euro-Dólar, filtrada de sábados e domingos e dos feriados nos Estados Unidos.

Uma vez corrigidos e filtrados os dados procedemos a obter o log-retorno do EuroDólar. A variável assim definida é uma variável fluxo. Sob estes retornos realizaremos as agregações temporais.
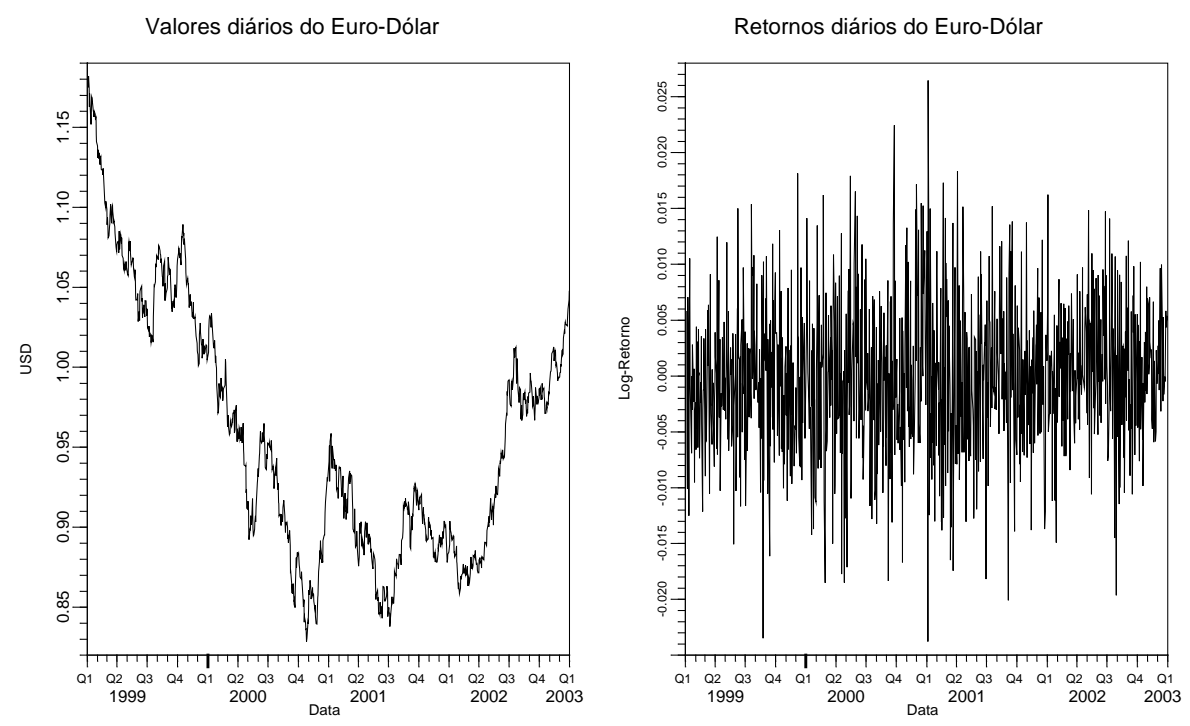

Ilustração 9.- Valores e retornos diários do Euro-Dólar.

Vamos fazer 10 agregações para variáveis fluxo: a primeira agregação, de 3 observações, corresponde a dados a cada 15 minutos; a segunda agregação, de 6 observações, corresponde a dados a cada 30 minutos; a terceira agregação, de 12 observações, corresponde a dados horários; a quarta agregação, de 24 observações, corresponde a dados a cada 2 horas; a quinta agregação, de 72 observações, corresponde a dados a cada 6 horas; a sexta agregação, de 288 observações, corresponde a dados diários; a sétima agregação, de 576 observações, corresponde a dados a cada 2 dias; a oitava agregação, de 1440 observações, corresponde a dados

${ }^{11}$ Este alisamento é no sentido de obter a série a cada 5 minutos a partir da série tick by tick, tomando a informação mais próxima anterior. Usamos a função align do S-Plus. 
semanais (5 dias úteis); a nona agregação, de 2880 observações, corresponde a dados quinzenais (10 dias úteis) e a última agregação, de 5760 observações, corresponde a dados mensais (20 dias úteis).

Usaremos a série de freqüência diária, como ponto de partida para termos o estudo de agregação e desagregação temporal.

Então, primeiramente obtemos os retornos da série com freqüência 5 minutos, e como em cada dia temos 288 observações, fazemos a agregação temporal das 288 observações considerando a definição de variáveis fluxo.

Na Ilustração 9 podem-se ver os valores diários da taxa de câmbio Euro-Dólar. Como pode-se notar, na série dos retornos pode-se ver certos agrupamentos destes tal como mencionamos nos fatos estilizados, embora seja difícil identificar quantos e onde começam e terminam.

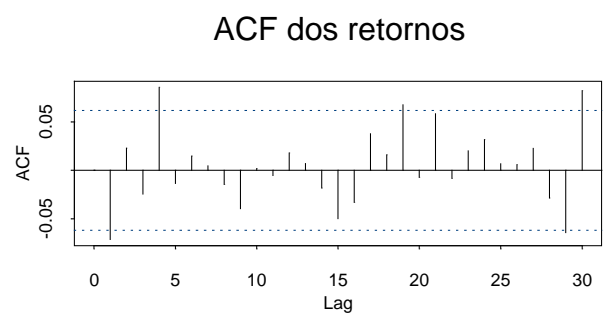

ACF dos retornos quadrados
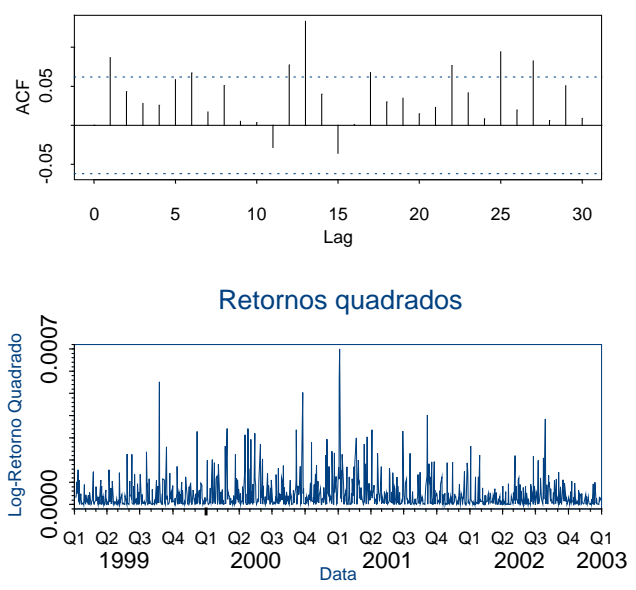

PACF dos retornos

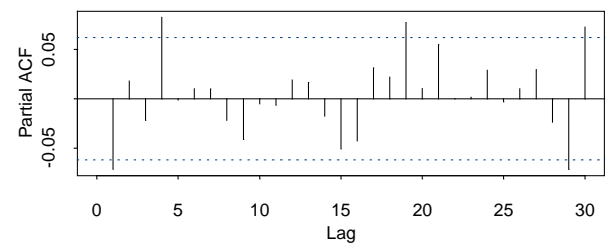

PACF dos retornos quadrados

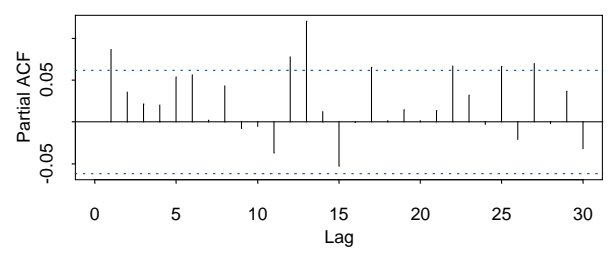

Histograma dos Retornos Quadrados

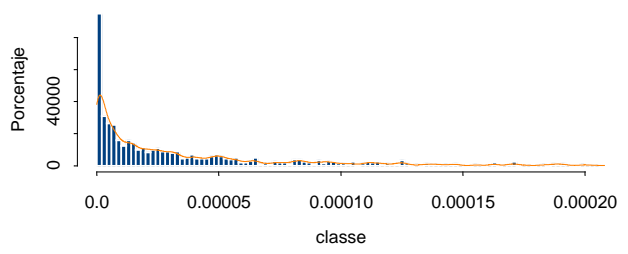

Ilustração 10.- Função de autocorrelação e autocorrelação parcial dos retornos e dos quadrados dos retornos do Euro-Dólar, plot e histograma dos quadrados dos retornos, frequiência diária.

Novamente, esta distinção entre os agrupamentos não é muito perceptível e pode ser melhor entendida quando observarmos os gráficos das funções de autocorrelação dos retornos e de seus quadrados, dado pela Ilustração 10. Nesta figura, vemos claramente que as autocorrelações de ordem 1, 4, etc. dos retornos é significante, rejeitando-se a hipótese de ruído branco e, portanto, violando o primeiro dos fatos estilizados. 
Podemos notar também que a primeira autocorrelação dos quadrados dos retornos é significante, mas após esta ordem a função de autocorrelação não decai lentamente, violando também o segundo fato estilizado.

Histograma dos Log-Retornos diários

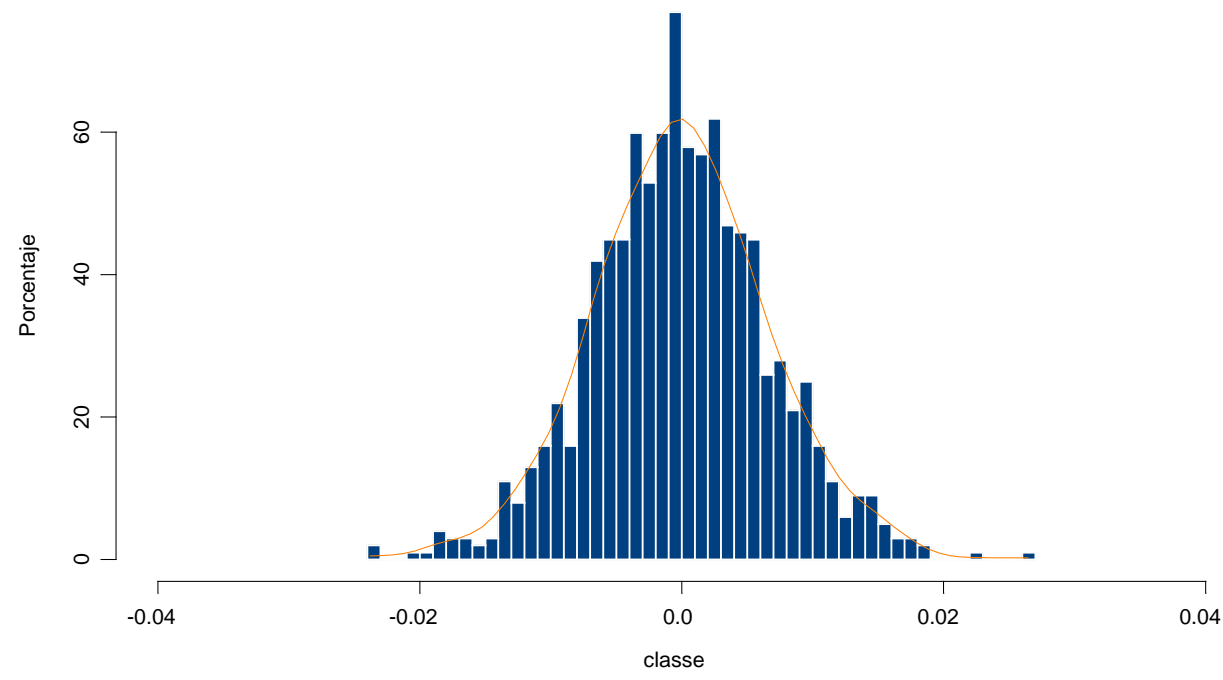

Ilustração 11.- Histograma dos retornos do Euro-Dólar, freqüência diária.

A Ilustração 11 apresenta o histograma dos retornos do Euro-Dólar e a estimação não paramétrica da densidade, com largura de faixa calculada segundo o método de validação cruzada não viciada.

Como tínhamos mencionado anteriormente, a presença de muitos eventos extremos faz com que o histograma pareça apresentar caudas pesadas, que validaria os fatos estilizados, mas poderíamos testar a normalidade dos retornos para poder confirmar efetivamente o fato estilizado que os retornos apresentam caudas pesadas em relação à distribuição normal.

Novamente, como no índice Ibovespa, existe também uma aparente simetria nos retornos. Mas novamente poderíamos estar enganados e a comprovação desta aparente simetria com um teste de hipótese é crucial, embora no mercado de FX não existe nenhum motivo, além do período selecionado, para ter um histograma assimétrico.

Uma das críticas que se tem quando se realizam testes de hipóteses com grandes bancos de dados é que estes não são robustos, no sentido de que pequenas diferenças rejeitam a hipótese nula.

O coeficiente de assimetria dos retornos é 0.22 e o coeficiente de curtose é 0.47 , e ao se testar hipóteses ao respeito da assimetria e de curtose, não se pode rejeitar a hipótese de não existir assimetria nem de existir um excesso de curtose em relação à distribuição normal, os p-valores da assimetria e curtose são 0.39 e 1 , respectivamente. 
No entanto, ao se testar a hipótese de normalidade, esta é rejeitada a um nível inferior a $0.01 \%$.

Os códigos com as análises descritivas para a série do Euro-Dólar foram feitos no SPlus.

\subsubsection{Estimação de modelos GARCH.}

O processo GARCH $(1,1)$ é:

$$
\sigma_{t}^{2}=\alpha_{0}+\alpha r_{t-1}^{2}+\beta \sigma_{t-1}^{2}
$$

onde $\sigma_{t}^{2}$ é a variância condicional e $r_{t}^{2}$ é a inovação quadrada.

Para provar os efeitos da heterogeneidade temporal dos mercados, este processo GARCH(1,1) é estimado para muitas freqüências. As mais baixas frequiências analisadas são mensais e as mais altas freqüências são definidas por uma série temporal homogênea com intervalos de 15 minutos para o índice Ibovespa e de 5 minutos para a taxa de câmbio Euro-Dólar. Nas mais altas frequiências incluiremos termos autoregressivos caso seja necessário, para considerar a significância (negativa) das autocorrelações dos retornos nestas frequiências. Também foi realizado um estudo sem a inclusão destes termos autoregressivos para não violar as hipóteses da agregação temporal.

Para a estimação dos parâmetros usaremos o método de máxima verossimilhança.

Seja $\theta$ o conjunto de parâmetros que caracterizam o processo. Assumimos que as inovações são normalmente distribuídas, então a log-verossimilhança é:

$$
l(\theta)=-\frac{n}{2} \ln (2 \pi)-\frac{1}{2} \sum_{t=1}^{n}\left(\ln \left(\sigma_{t}^{2}\right)+\frac{r_{t}^{2}}{\sigma_{t}^{2}}\right)
$$

O procedimento de log-verossimilhança tem importantes propriedades. A solução é independente do sistema de coordenadas onde os parâmetros são definidos, assim que a estimação pode ser feita em qualquer parametrização e os resultados serão idênticos, para a transformação de parâmetros que seja escolhida. Esta propriedade é verdadeira para amostras finitas e qualquer conjunto de dados, assumindo um máximo não degenerado. Até mesmo se o processo é mal especificado (i.e. os dados não forem gerados pelo processo estimado) o máximo é idêntico em qualquer sistema de coordenadas.

A suposição de normalidade condicional para as inovações pode ser trocada pela suposição de uma distribuição t-Student ou a distribuição exponencial generalizada. Estas duas últimas distribuições têm caudas mais pesadas que a distribuição normal. 
No caso da distribuição t-Student, a função de log-verossimilhança tem a seguinte forma:

$$
\begin{aligned}
l(\theta) & =-\frac{n}{2}\left[\ln (v-2)+2 \ln \left(\pi^{1 / 2} \Gamma\left(\frac{v}{2}\right)\right)-\ln \Gamma\left(\frac{v+1}{2}\right)\right] \\
& -\frac{1}{2} \sum_{t=1}^{n}\left[\ln \left(\sigma_{t}^{2}\right)+(v+1) \ln \left(1+\frac{r_{t}^{2}}{\sigma_{t}^{2}(v-2)}\right)\right],
\end{aligned}
$$

onde $v$ é o número de graus de liberdade da distribuição $t$-Student e $\Gamma$ é a função gama.

O máximo da função de verossimilhança é encontrado usando o algoritmo Berndt, Hall, Hall e Hausman (BHHH) (Berndt et al., 1974) o qual é uma variante do método do gradiente.

Estimar processos GARCH por máxima verossimilhança é difícil porque a função de verossimilhança é grande e quase constante em grande parte do espaço paramétrico, veja Zumbach (2000). Por este motivo têm surgido alternativas como o estimador de quase máxima verossimilhança e os estimadores Bayesianos.

Weiss (1986) e Bollerslev e Wooldridge (1992), mostraram que o estimador de quase máxima verossimilhança, QMLE, de um processo GARCH forte com distribuição normal das inovações é consistente se a variância está corretamente especificada. Este resultado não se aplica quando a distribuição das inovações é $t$-student.

Mais adiante, na estimação dos processos HARCH, além do método de máxima verossimilhança, usaremos o algoritmo do amostrador de Gibbs para a estimação e comparação dos parâmetros estimados.

Como já tínhamos mencionado, os dados empíricos podem ser descritos como gerados por um processo $\operatorname{GARCH}(1,1)$ em uma particular frequiência. O comportamento dos dados amostrais em qualquer outra frequiência é teoricamente determinado pela agregação (ou desagregação) temporal do processo original dados pela proposição 2.1. Estes processos derivados teoricamente em diferentes freqüências podem ser comparados aos processos estimados empiricamente nas mesmas frequiências.

Diferenças significativas entre os resultados empíricos e teóricos conduzem à rejeição da hipótese de somente um processo GARCH gerar os dados. Então podemos mostrar que há mais que uma frequiência relevante na geração da volatilidade, então o mercado pode ser chamado, por enquanto, heterogêneo.

Dado que resultados anteriores confirmaram a adequação destes resultados teóricos nas freqüências menores que 1 hora (Drost e Nijman, 1993), nós usaremos as estimações diárias e de freqüência maior como um ponto de partida para calcular os resultados para as mais altas freqüências mas também para fazer a agregação temporal para as baixas frequiências.

Altas freqüências têm, também, a vantagem de alto nível de significância estatística. 
Ao explorar a agregação temporal, temos que escolher uma escala de tempo. Sazonalidade não é a matéria de um estudo de agregação, mas poderia incomodar. Eliminar as sazonalidades usando uma escala de tempo diferente ao tempo físico poderia ajudar nos resultados. A escala que usaremos, a qual chamaremos de escala de tempo de negócios, simplesmente omite os períodos de final de semana, feriados ou quando os mercados são virtualmente fechados, tal como foi detalhado anteriormente. No tempo físico os finais de semana cobrem dois sétimos da amostra total. Isto causa um completo colapso no processo de estimação produzindo estimações de $\alpha$ muito grandes. O tempo físico incluindo os finais de semana é simplesmente impossível de usar aqui.

\subsubsection{Estimações e agregação temporal na série do Ibovespa.}

Partimos da série de tempo do índice Ibovespa diária e realizamos a agregação e desagregação temporal, usando a proposição 2.1, para as freqüências 15 minutos, 1.25 horas, 1 semana e 1 mês. Em outras palavras vamos fazer agregações de 5 (semana) e 20 (mês) retornos e desagregações de ordem 25 (15 minutos) e 5 (1,25 horas).

Para todas estas freqüências realizamos, também, a estimação dos coeficientes GARCH(1,1) com e sem introdução de uma filtragem através de uma componente autoregressiva na equação da média.
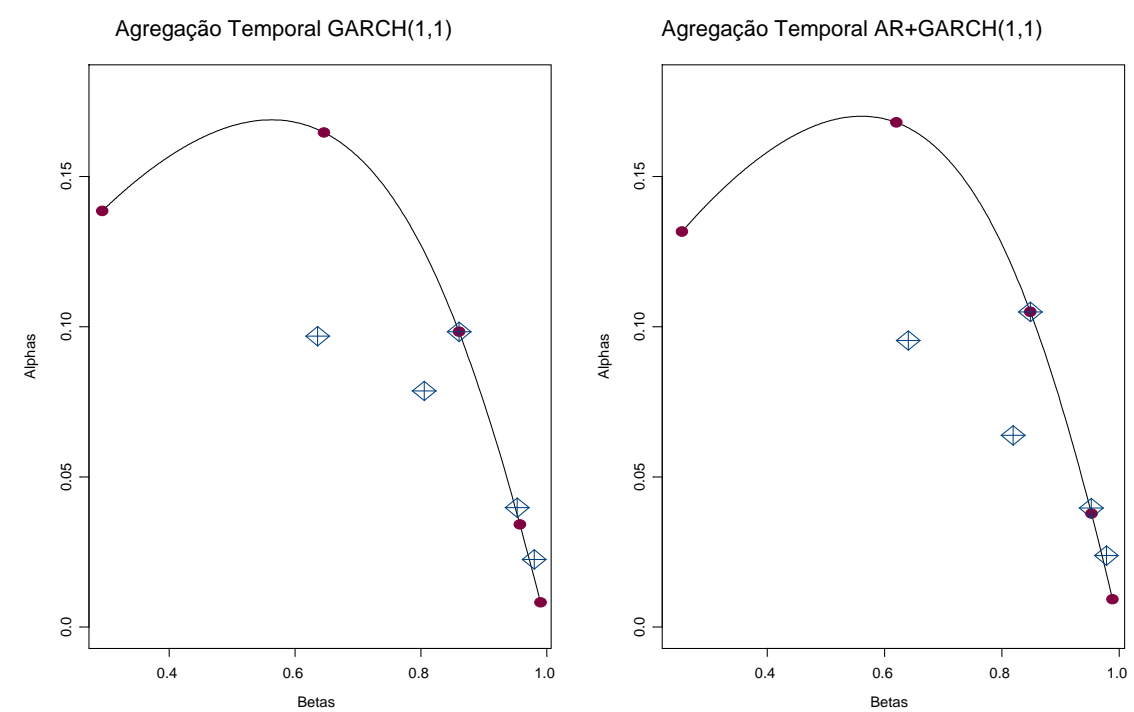

Ilustração 12.- Agregação temporal do $\operatorname{GARCH}(1,1)$ na escala de tempo de negócios.

Os coeficientes $\alpha$ e $\beta$ tanto teóricos (proposição 2.1) como empíricos (estimações de máxima verossimilhança) são mostrados na Ilustração 12 e na Tabela 3. Os coeficientes teóricos são mostrados pelos círculos e os estimados pelos diamantes. 


\begin{tabular}{|c|c|c|c|c|c|c|}
\hline \multicolumn{4}{|c|}{$\operatorname{GARCH}(1,1)$} & \multicolumn{3}{|c|}{ Teóricos } \\
\hline Freqüência & Parâmetro & Estimações & Erro padrão & Freqüência & Parâmetro & Agregação \\
\hline 15 minutos & $\begin{array}{l}\text { Curtose } \\
\text { Alpha } 0 \\
\text { Alpha } \\
\text { Beta }\end{array}$ & $\begin{array}{c}49.33 \\
1.04 \mathrm{E}-07 \\
0.02251 \\
0.98005\end{array}$ & $\begin{array}{l}1.24 \mathrm{E}-08 \\
0.00199 \\
0.00117\end{array}$ & 15 minutos & $\begin{array}{l}\text { Curtose } \\
\text { Alpha } 0 \\
\text { Alpha } \\
\text { Beta }\end{array}$ & $\begin{array}{c}94.25 \\
3.76 \mathrm{E}-08 \\
0.00818 \\
0.99014\end{array}$ \\
\hline \multirow[b]{2}{*}{1,25 horas } & Curtose & 16.48 & & \multirow[b]{2}{*}{1,25 horas } & Curtose & 27.41 \\
\hline & $\begin{array}{l}\text { Alpha } 0 \\
\text { Alpha } \\
\text { Beta }\end{array}$ & $\begin{array}{l}1.09 E-06 \\
0.03973 \\
0.95292\end{array}$ & $\begin{array}{c}2.23 E-07 \\
0.00509 \\
0.00524\end{array}$ & & $\begin{array}{l}\text { Alpha } 0 \\
\text { Alpha } \\
\text { Beta }\end{array}$ & $\begin{array}{c}9.38 \mathrm{E}-07 \\
0.03411 \\
0.95753\end{array}$ \\
\hline 1 dia & $\begin{array}{l}\text { Curtose } \\
\text { Alpha } 0 \\
\text { Alpha } \\
\text { Beta } \\
\end{array}$ & $\begin{array}{c}13.96 \\
2.31 \mathrm{E}-05 \\
0.09831 \\
0.86062 \\
\end{array}$ & $\begin{array}{c}7.66 \mathrm{E}-06 \\
0.01992 \\
0.02745 \\
\end{array}$ & 1 dia & $\begin{array}{l}\text { Curtose } \\
\text { Alpha } 0 \\
\text { Alpha } \\
\text { Beta } \\
\end{array}$ & $\begin{array}{c}13.96 \\
2.31 \mathrm{E}-05 \\
0.09831 \\
0.86062 \\
\end{array}$ \\
\hline \multirow[t]{2}{*}{1 semana } & $\begin{array}{l}\text { Curtose } \\
\text { Alpha } 0 \\
\text { Alpha }\end{array}$ & $\begin{array}{c}1.43 \\
3.28 \mathrm{E}-04 \\
0.07863\end{array}$ & $\begin{array}{c}3.18 \mathrm{E}-04 \\
0.05935\end{array}$ & \multirow[t]{2}{*}{1 semana } & $\begin{array}{l}\text { Curtose } \\
\text { Alpha } 0 \\
\text { Alpha }\end{array}$ & $\begin{array}{c}10.88 \\
5.31 \mathrm{E}-04 \\
0.16470\end{array}$ \\
\hline & Beta & 0.80513 & 0.14840 & & Beta & 0.64613 \\
\hline 1 mês & $\begin{array}{l}\text { Curtose } \\
\text { Alpha } 0 \\
\text { Alpha } \\
\text { Beta }\end{array}$ & $\begin{array}{c}2.16 \\
3.41 \mathrm{E}-03 \\
0.09688 \\
0.63616\end{array}$ & $\begin{array}{c}8.60 \mathrm{E}-03 \\
0.19399 \\
0.79153\end{array}$ & 1 mês & $\begin{array}{l}\text { Curtose } \\
\text { Alpha } 0 \\
\text { Alpha } \\
\text { Beta }\end{array}$ & $\begin{array}{c}9.12 \\
6.38 \mathrm{E}-03 \\
0.13856 \\
0.29368\end{array}$ \\
\hline
\end{tabular}

Tabela 3.- Agregação temporal do GARCH(1,1) na escala de tempo de negócios.

Devido ao fato de caudas pesadas dos retornos, a distribuição considerada para a estimação de máxima verossimilhança foi a $t$ de student.

Em ambos os gráficos pode-se comprovar que a hipótese de que a volatilidade tenha sido gerada por somente um processo $\operatorname{GARCH}(1,1)$ é rejeitada, mesmo quando incluímos termos autoregressivos na equação das médias.

\subsubsection{Estimações e agregação temporal na série do EURO.}

Partimos da série de tempo da taxa de câmbio Euro-Dólar com freqüência de 2 horas e realizamos a agregação e desagregação temporal, usando a proposição 2.1, para as frequiências 5 minutos, 15 minutos, 30 minutos, 1 hora, 2 horas, 1 dia, 2 dias, 1 semana, 2 semanas e 1 mês. Em outras palavras vamos fazer agregações de 4 (1 dia), 8 (2 dias), 20 (1 semana), 40 (2 semanas) e 80 (1 mês) retornos, e desagregações de ordem 72 (5 minutos), 24 (15 minutos), 12 (trinta minutos), 6 (uma hora) e 3 (2 horas). Para todas estas frequiências realizamos, também, a estimação dos coeficientes GARCH(1,1) com e sem introdução de uma filtragem através de uma parte autoregressiva na equação da média. 


\begin{tabular}{|c|c|c|c|c|c|c|}
\hline \multicolumn{4}{|c|}{$\operatorname{GARCH}(1,1)$} & \multicolumn{3}{|c|}{ Teóricos } \\
\hline Freqüência & Parâmetro & Estimações & Erro padrão & Freqüência & Parâmetro & Agregação \\
\hline \multirow{4}{*}{5 minutos } & Curtose & 71.28 & & \multirow{4}{*}{5 minutos } & Curtose & -32.8702 \\
\hline & Alpha 0 & $7.98 \mathrm{E}-13$ & $4.88 \mathrm{E}-14$ & & Alpha 0 & $3.85 \mathrm{E}-11$ \\
\hline & Alpha & 0.00000 & 0.00000 & & Alpha & 0 \\
\hline & Beta & 0.99999 & 0.00000 & & Beta & 1 \\
\hline \multirow{4}{*}{15 minutos } & Curtose & 52.53 & & \multirow{4}{*}{15 minutos } & Curtose & -3.8912 \\
\hline & Alpha 0 & $3.14 \mathrm{E}-08$ & 1.05E-09 & & Alpha 0 & $3.47 \mathrm{E}-10$ \\
\hline & Alpha & 0.25358 & 0.00629 & & Alpha & 0.017813 \\
\hline & Beta & 0.75062 & 0.00417 & & Beta & 0.982114 \\
\hline \multirow{4}{*}{30 minutos } & Curtose & 17.02 & & \multirow{4}{*}{30 minutos } & Curtose & 3.353427 \\
\hline & Alpha 0 & 1.39E-07 & 6.38E-09 & & Alpha 0 & 1.39E-09 \\
\hline & Alpha & 0.34563 & 0.01410 & & Alpha & 0.012914 \\
\hline & Beta & 0.64414 & 0.00861 & & Beta & 0.986941 \\
\hline \multirow{4}{*}{1 hora } & Curtose & 8.51 & & \multirow{4}{*}{1 hora } & Curtose & 6.975536 \\
\hline & Alpha 0 & 1.92E-08 & 3.13E-09 & & Alpha 0 & $5.55 \mathrm{E}-09$ \\
\hline & Alpha & 0.02711 & 0.00266 & & Alpha & 0.017435 \\
\hline & Beta & 0.97240 & 0.00233 & & Beta & 0.98235 \\
\hline \multirow{4}{*}{2 horas } & Curtose & 8.79 & & \multirow{4}{*}{2 horas } & Curtose & 8.786181 \\
\hline & Alpha 0 & 2.22E-08 & 5.76E-09 & & Alpha 0 & 2.22E-08 \\
\hline & Alpha & 0.01975 & 0.00272 & & Alpha & 0.019753 \\
\hline & Beta & 0.97982 & 0.00250 & & Beta & 0.979816 \\
\hline \multirow{4}{*}{6 horas } & Curtose & 3.90 & & \multirow{4}{*}{6 horas } & Curtose & 9.99146 \\
\hline & Alpha 0 & $6.00 \mathrm{E}-08$ & 3.18E-08 & & Alpha 0 & 2E-07 \\
\hline & Alpha & 0.01954 & 0.00475 & & Alpha & 0.030075 \\
\hline & Beta & 0.97934 & 0.00488 & & Beta & 0.968633 \\
\hline \multirow{4}{*}{1 dia } & Curtose & 0.47 & & \multirow{4}{*}{1 dia } & Curtose & 10.43445 \\
\hline & Alpha 0 & 4.49E-07 & 4.53E-07 & & Alpha 0 & $3.19 \mathrm{E}-06$ \\
\hline & Alpha & 0.02659 & 0.01118 & & Alpha & 0.054788 \\
\hline & Beta & 0.96347 & 0.01807 & & Beta & 0.940053 \\
\hline \multirow{4}{*}{2 dias } & Curtose & 0.43 & & \multirow{4}{*}{2 dias } & Curtose & 10.49687 \\
\hline & Alpha 0 & $8.18 \mathrm{E}-07$ & $1.38 \mathrm{E}-06$ & & Alpha 0 & $1.27 \mathrm{E}-05$ \\
\hline & Alpha & 0.01952 & 0.01502 & & Alpha & 0.073334 \\
\hline & Beta & 0.97034 & 0.02662 & & Beta & 0.916376 \\
\hline \multirow{4}{*}{1 semana } & Curtose & -0.21 & & \multirow{4}{*}{1 semana } & Curtose & 10.5032 \\
\hline & Alpha 0 & 9.38E-06 & $1.51 \mathrm{E}-05$ & & Alpha 0 & $7.89 \mathrm{E}-05$ \\
\hline & Alpha & 0.06008 & 0.04523 & & Alpha & 0.104596 \\
\hline & Beta & 0.88069 & 0.11135 & & Beta & 0.869876 \\
\hline \multirow{4}{*}{2 semanas } & Curtose & -0.03 & & \multirow{4}{*}{2 semanas } & Curtose & 10.45405 \\
\hline & Alpha 0 & 4.06E-05 & $1.64 \mathrm{E}-04$ & & Alpha 0 & 0.000312 \\
\hline & Alpha & 0.03252 & 0.08858 & & Alpha & 0.131723 \\
\hline & Beta & 0.85182 & 0.51501 & & Beta & 0.817874 \\
\hline \multirow{4}{*}{1 mês } & Curtose & 0.30 & & & Curtose & 10.33557 \\
\hline & Alpha 0 & $1.43 \mathrm{E}-04$ & $3.25 E-03$ & $1 \mathrm{~m}$ & Alpha 0 & 0.001215 \\
\hline & Alpha & 0.01110 & 0.15955 & 11 mes & Alpha & 0.157582 \\
\hline & Beta & 0.81428 & 4.04681 & & Beta & 0.744151 \\
\hline
\end{tabular}

Tabela 4.- Agregação temporal do $\operatorname{GARCH}(1,1)$ na escala de tempo de negócios. 

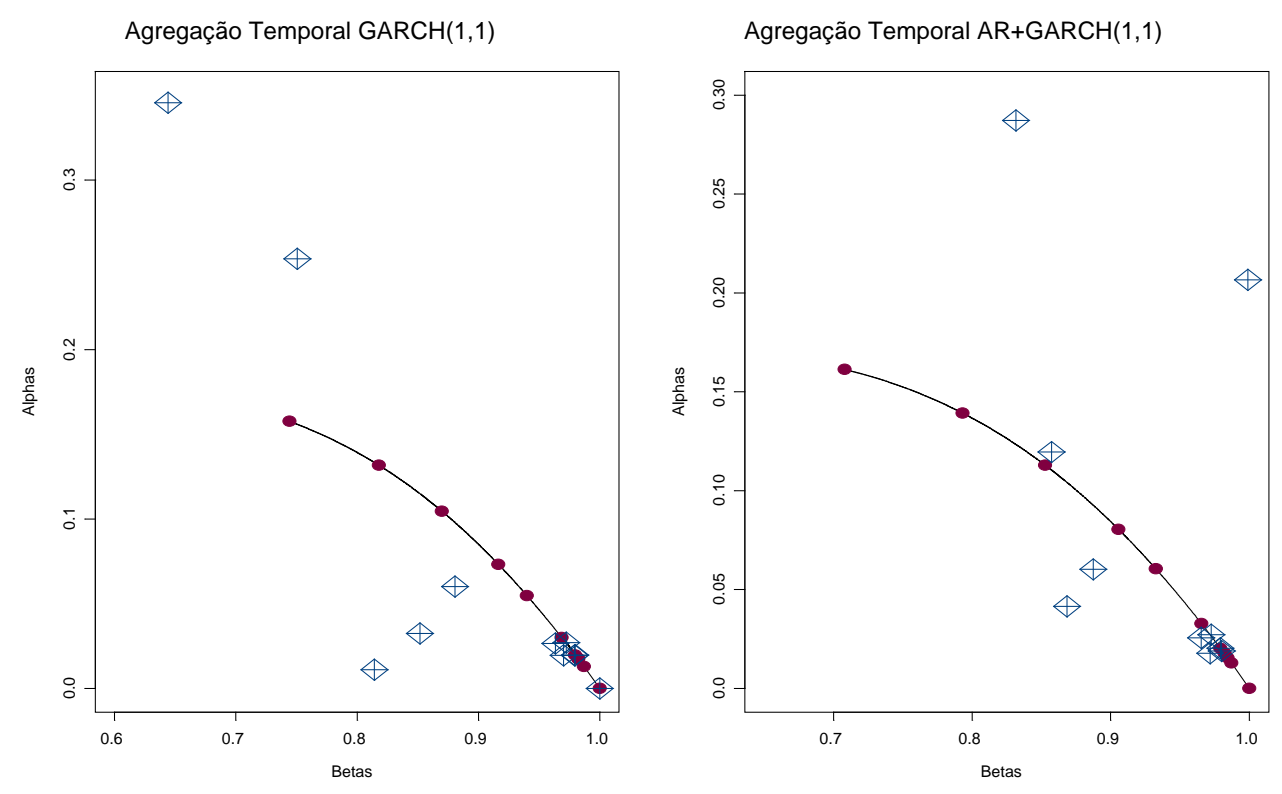

Ilustração 13.- Agregação temporal do $\operatorname{GARCH}(1,1)$ na escala de tempo de negócios.

Os coeficientes $\alpha$ e $\beta$ tanto teóricos (proposição 2.1) como empíricos (estimações de máxima verossimilhança) são mostrados na Ilustração 13 e na Tabela 4. Os coeficientes teóricos são mostrados pelos círculos e os estimados pelos diamantes.

Devido ao fato de caudas pesadas dos retornos, a distribuição considerada para a estimação de máxima verossimilhança é a $t$ de student.

Em ambos gráficos pode-se comprovar que a hipótese de que a volatilidade tenha sido gerada por somente um processo $\operatorname{GARCH}(1,1)$ é rejeitada, mesmo quando incluímos termos autoregressivos na equação da média.

\subsubsection{Estabilidade Temporal.}

Tanto as séries do IBOVESPA quanto a do EURO apresentam agrupamentos, "clusters", de volatilidade que têm aproximadamente o mesmo tamanho para todos os níveis de agregação. Em outras palavras a memória da volatilidade parece ter uma vida mais curta em alta freqüência e uma vida mais longa para freqüências maiores, portanto a informação contida da volatilidade não é a mesma para diferentes frequiências. Alguns autores atribuem isto à presença de muitas componentes de volatilidade independentes nos dados, ou seja, a característica de heterogeneidade do mercado. O modelo GARCH não captura a heterogeneidade dos negociadores que atuam em diferentes horizontes de tempo. 
Estabilidade Temporal do Ibovespa

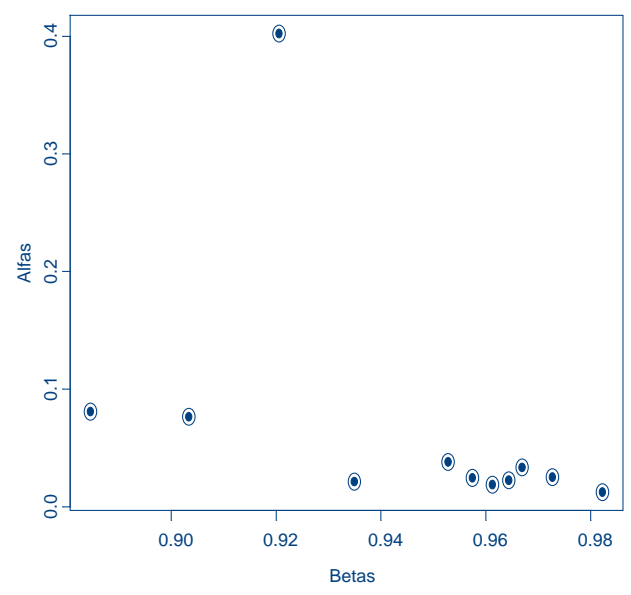

Estabilidade Temporal do Euro

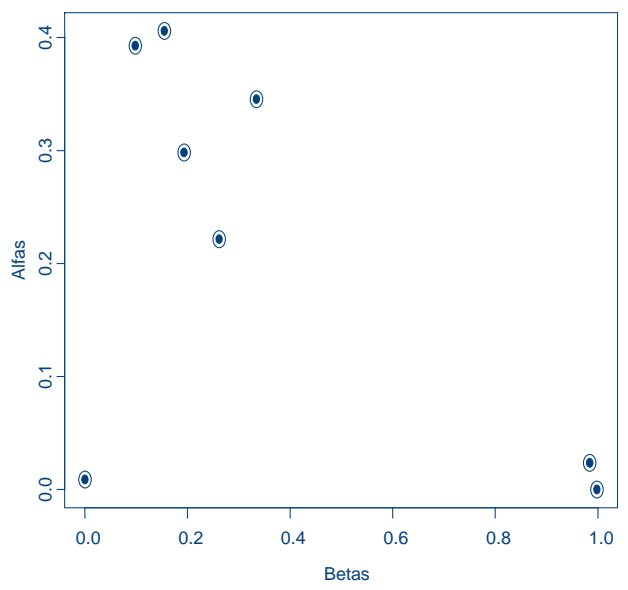

Ilustração 14.- Parâmetros estimados do $\operatorname{GARCH}(1,1)$ para sub-amostras de 6 meses.

Esta é uma explicação razoável dos resultados obtidos em altas frequiências quando usamos um modelo GARCH com uma distribuição $t$-student para os retornos.

Para avaliar o comportamento da volatilidade estimada por um processo $\operatorname{GARCH}(1,1)$ investigamos a estabilidade temporal dos coeficientes estimados para várias subamostras.

Realizamos a estimação dos parâmetros $\operatorname{GARCH}(1,1)$ para Ibovespa e AR+GARCH(1,1) para o Euro-Dólar em intervalos de tempo de 1,25 e 2 horas, respectivamente.

Para isso dividimos as séries em sub-séries de 6 meses, com cerca de 626 observações por série para o Ibovespa e 1504 observações para o Euro.

Como podemos ver na Ilustração 14 os coeficientes não são estáveis no tempo. Alguns deles ficam à esquerda do gráfico e muito longe dos outros. Além disso, estes coeficientes aberrantes não estão ligados com alguma seqüência temporal. As mudanças nos valores dos coeficientes têm uma seqüência irregular no tempo. A hipótese de que todos os parâmetros são iguais pode ser rejeitada com uma alta significância se usamos o teste de razão de verossimilhança (Hamilton, 1994). Novamente isto é um sinal da má especificação do modelo, mas poderia também indicar mudanças no comportamento do mercado.

Um tamanho crescente da amostra não melhora as previsões da volatilidade pelo modelo GARCH. A qualidade da previsão se satura quando incrementamos o tamanho da amostra depois de certo limite (threshold). As sub-amostras usadas são suficientemente grandes, assim o comportamento errôneo dos coeficientes GARCH estimados não pode ser atribuído a tamanhos de amostras muito pequenas. 


\section{Capítulo 3}

\section{Modelos ARCH Heterogêneos}

\section{(HARCH)}

\subsection{Introdução.}

Dacorogna, et al. (2001) mostram que há uma assimetria na interação entre as volatilidades medidas em diferentes frequiências. Uma volatilidade "grossa" prediz uma volatilidade "fina" melhor do que o contrário. Como este efeito não está presente num simples modelo GARCH foram desenvolvidos alguns tipos mais complexos de modelos ARCH para assim esclarecer a heterogeneidade encontrada nos dados de alta freqüência. Um destes é o modelo HARCH (Heterogeneous Autoregressive Conditional Heteroskedasticity).

O processo HARCH tem uma variação da equação da variância, pois esta é baseada nos retornos sobre intervalos de tamanhos diferentes. O comportamento empírico da correlação retardada, dado pela heterogeneidade da informação nos mercado financeiros, pode ser reproduzido bem com este novo processo, e ao mesmo tempo, HARCH pode reproduzir a memória longa da volatilidade, como visto em Dacorogna et al. (1993), e Ding et al. (1993). Além disso, como veremos mais à frente, os termos da variância condicional do HARCH refletem a estrutura heterogênea do mercado de uma maneira natural. 


\subsection{O modelo HARCH.}

O modelo $\operatorname{HARCH}(n)$ devido a Müller et al. (1997) é dado por:

$$
\begin{aligned}
& r_{t}=\sigma_{t} \varepsilon_{t}, \\
& \sigma_{t}^{2}=c_{0}+\sum_{j=1}^{n} c_{j}\left(\sum_{i=1}^{j} r_{t-i}\right)^{2},
\end{aligned}
$$

onde $c_{0}>0, c_{n}>0, c_{j} \geq 0 \forall j=1, \ldots, n-1$ e os $\varepsilon_{t}$ são variáveis aleatórias i.i.d. com média zero e variância um.

A equação para a variância $\sigma_{t}^{2}$ é uma combinação linear dos quadrados dos retornos agregados para assim poder eliminar o problema da heterogeneidade da informação incluída nos dados.

$\mathrm{O}$ processo HARCH pertence à família $\mathrm{ARCH}$, mas difere de todos os outros processos tipo ARCH, na importante propriedade de considerar as volatilidades dos retornos medidas em intervalos de diferentes tamanhos. O processo ARCH Quadrático (QARCH) (veja Sentana, 1991) é uma exceção e pode ser considerado como uma forma generalizada de HARCH.

Para ilustrar algumas características especiais dos processos HARCH podemos considerar o HARCH(2). A equação da variância do HARCH(2) pode ser escrita como segue:

$$
\begin{aligned}
\sigma_{t}^{2} & =c_{0}+c_{1} r_{t-1}^{2}+c_{2}\left(r_{t-1}+r_{t-2}\right)^{2} \\
& =c_{0}+\left(c_{1}+c_{2}\right) r_{t-1}^{2}+c_{2} r_{t-2}^{2}+2 c_{2} r_{t-1} r_{t-2} .
\end{aligned}
$$

Como podemos ver, este processo HARCH(2) pode ser identificado como um processo ordinário $\mathrm{ARCH}(2)$, com exceção de termo que contém o produto misto $r_{t-1} r_{t-2}$. Nos processos tipo ARCH, só os valores absolutos dos retornos são importantes, entretanto, no HARCH, o sinal dos retornos é também importante. Dois retornos subseqüentes do mesmo tamanho e nas mesmas direções causarão uma contribuição mais alta à variância do processo que dois retornos subseqüentes com sinais opostos.

Como sabemos, a volatilidade tem memória longa, portanto, nós precisamos de uma ordem alta no HARCH, um $n$ grande, para modelar o comportamento das séries de tempo empíricas. Isto implica um número alto de coeficientes $c_{j}$.

Portanto precisa-se de uma parametrização parcimoniosa. No caso do $\mathrm{ARCH}$, alguns processos de alta ordem podem ser formulados como processos GARCH de baixa ordem, veja Bollerslev (1986), mas são os processos FIGARCH, veja Baillie et al. (1996), os que resolvem o problema da memória longa. 


\subsection{Extensões do modelo HARCH.}

$\mathrm{Na}$ formulação dos processos $\mathrm{HARCH}$, os coeficientes $c_{1}, \ldots, c_{n}$ poderiam não ser considerados parâmetros livres no modelo, com o objetivo de obter uma aproximação parcimoniosa, veja Dacorogna et al. (1993), e Ding et al. (1993).

Vamos trabalhar com uma aproximação parcimoniosa diferente da proposta destes autores. Esta aproximação parcimoniosa do mercado heterogêneo conduz a um baixo número de parâmetros livres no modelo, que determinam um número muito maior $n$ de coeficientes que modelam a memória longa da volatilidade.

Esta parametrização parcimoniosa permite a exploração da estrutura dos componentes do mercado. Os coeficientes $c_{j}$ refletem o impacto relativo de componentes de mercado diferentes com intervalos de tempo pertinentes diferentes. Então, selecionamos $m$ componentes do mercado que correspondem a $m$ parâmetros livres, cada um associado a algum coeficiente $c_{j}$.

Em outras palavras temos um modelo $\operatorname{HARCH}(n)$ parcimonioso com $n=a^{m}$, com $m$ agregações de tamanhos diferentes $a^{0}, \ldots, a^{m}$, se,

$$
\begin{aligned}
r_{t} & =\sigma_{t} \varepsilon_{t}, \\
\sigma_{t}^{2} & =C_{0}+C_{1} r_{t-1}^{2}+C_{2}\left(r_{t-1}+\ldots+r_{t-a}\right)^{2}+\ldots+C_{m}\left(r_{t-1}+\ldots+r_{t-a^{m}}\right)^{2},
\end{aligned}
$$

onde $\varepsilon_{t} \sim$ i.i.d. $(0,1)$ e $C_{0}>0, \quad C_{j} \geq 0, \quad \forall j=1, \ldots, m-1, \quad C_{m}>0{ }^{12}$.

Aliás, podemos generalizar mais ainda se consideramos um modelo $\operatorname{HARCH}(m)$ parcimonioso com agregações de tamanhos diferentes $a_{1}, \ldots, a_{m}$, onde $m$ é o número de componentes do mercado se,

$$
\begin{aligned}
r_{t} & =\sigma_{t} \varepsilon_{t}, \\
\sigma_{t}^{2} & =C_{0}+C_{1}\left(r_{t-1}+\ldots+r_{t-a_{1}}\right)^{2}+\ldots+C_{m}\left(r_{t-1}+\ldots+r_{t-a_{m}}\right)^{2},
\end{aligned}
$$

onde $\varepsilon_{t} \sim$ i.i.d. $(0,1)$ e $C_{0}>0, \quad C_{j} \geq 0, \quad \forall j=1, \ldots, m-1, \quad C_{m}>0$.

Note-se que em todas estas equações podemos também introduzir uma equação de média, quando os retornos são autocorrelacionados.

Também, do mesmo modo que os processos GARCH generalizam os processos $\mathrm{ARCH}$, podemos generalizar os processos $\mathrm{HARCH}$, assim:

12 Note-se que usamos letras maiúsculas nos coeficientes dos modelos $\operatorname{HARCH}(n)$ parcimonioso, para não confundí-los com os coeficientes do modelo $\operatorname{HARCH}(n)$ simples (não parcimonioso). 


$$
\begin{aligned}
& r_{t}=\sigma_{t} \varepsilon_{t}, \\
& \sigma_{t}^{2}=c_{0}+\sum_{j=1}^{n} \sum_{k=1}^{j} c_{j k}\left(\sum_{i=k}^{j} r_{t-i}\right)^{2}+\sum_{i=1}^{p} b_{i} \sigma_{t-i}^{2},
\end{aligned}
$$

onde $c_{0}>0, \quad c_{j k} \geq 0, \quad \forall j=1, \ldots, n-1, \quad k=1, \ldots, j, \quad b_{i} \geq 0, \quad \forall i=1, \ldots, p$.

Esta equação generalizada do processo considera todos os retornos entre qualquer par de pontos de tempo no período entre $t-n$ e $t$-1. Esta equação cobre os processos HARCH, ARCH e GARCH. Embora, ao introduzir este último termo na equação da variância, (um termo GARCH), podemos contribuir para uma formulação parcimoniosa do modelo, podemos também perder a oportunidade de indicar claramente a estrutura das componentes atuais, isto é, dos diferentes agrupamentos dos retornos necessários.

Também nos processos HARCH parcimoniosos podemos realizar a mesma generalização com a introdução de um termo GARCH na equação de variância. Assim, por exemplo, o processo $\operatorname{GHARCH}(n, p)$ parcimonioso com $n=a^{m-1}$, onde $m$ é o número de componentes do mercado, é dado por

$$
\begin{aligned}
r_{t}= & \sigma_{t} \varepsilon_{t}, \\
\sigma_{t}^{2}= & C_{0}+C_{1} r_{t-1}^{2}+C_{2}\left(r_{t-1}+\ldots+r_{t-a}\right)^{2}+\ldots+C_{m+1}\left(r_{t-1}+\ldots+r_{t-a^{m}}\right)^{2}+ \\
& +b_{1} \sigma_{t-1}^{2}+\ldots+b_{p} \sigma_{t-p}^{2},
\end{aligned}
$$

onde $\quad C_{j} \geq 0, \quad j=0, \ldots, m-1, \quad C_{m}>0, \quad b_{i} \geq 0, \quad i=1, \ldots, p-1, \quad b_{p}>0 \quad \mathrm{e}$ $\varepsilon_{t} \sim$ i.i.d. $(0,1)$.

Por último, o processo $\operatorname{GHARCH}(m, p)$ parcimonioso com agregações de tamanhos diferentes $a_{1}, \ldots, a_{m}$, onde $m$ é o número de componentes do mercado, é dado por

$$
\begin{aligned}
r_{t}= & \sigma_{t} \varepsilon_{t} \\
\sigma_{t}^{2} & =C_{0}+C_{1}\left(r_{t-1}+\ldots+r_{t-a_{1}}\right)^{2}+\ldots+C_{m}\left(r_{t-1}+\ldots+r_{t-a_{m}}\right)^{2}+ \\
& +b_{1} \sigma_{t-1}^{2}+\ldots+b_{p} \sigma_{t-p}^{2}
\end{aligned}
$$

onde $\quad C_{j} \geq 0, \quad j=0, \ldots, m-1, \quad C_{m}>0, \quad b_{i} \geq 0, \quad i=1, \ldots, p-1, \quad b_{p}>0 \quad \mathrm{e}$ $\varepsilon_{t} \sim$ i.i.d. $(0,1)$.

Algumas outras variantes dos modelos HARCH têm sido desenvolvidas, como por exemplo, os processos EMA-HARCH (Dacorogna et. al, 2001). A principal idéia do $\mathrm{HARCH}$, isto é, fazer agregações de diferentes tamanhos, pode ser combinada com 
algumas extensões dos modelos ARCH, como por exemplo, M-GARCH, e assim obter variantes dos processos HARCH.

Agora vamos demonstrar algumas propriedades dos processos HARCH. Condições necessárias e suficientes para a estacionariedade dos processos HARCH mais simples são dadas por Dacorogna et al. (1996). Estenderemos estes resultados e mostraremos alguns outros para os processos HARCH generalizados e parcimoniosos.

\subsection{Estacionariedade. Condições Necessárias.}

Nesta seção obteremos condições necessárias de estacionariedade para os modelos HARCH.

\section{HARCH (2)}

$$
\begin{aligned}
& r_{t}=\sigma_{t} \varepsilon_{t}, \\
& \sigma_{t}^{2}=c_{0}+c_{1} r_{t-1}^{2}+c_{2}\left(r_{t-1}+r_{t-2}\right)^{2},
\end{aligned}
$$

$\varepsilon_{t} \sim$ i.i.d. $(0,1)$.

Se $r_{t}$ é estacionário temos:

$$
E\left(r_{t}^{2}\right)=E\left(r_{t-i}^{2}\right), \forall i \geq 1
$$

Então,

$$
\begin{aligned}
E\left(r_{t}^{2}\right) & =E\left(\sigma_{t}^{2} \varepsilon_{t}^{2}\right)=E\left(\sigma_{t}^{2}\right) E\left(\varepsilon_{t}^{2}\right)=E\left(\sigma_{t}^{2}\right) \\
& =E\left(c_{0}+c_{1} r_{t-1}^{2}+c_{2}\left(r_{t-1}+r_{t-2}\right)^{2}\right) \\
& =c_{0}+\left(c_{1}+c_{2}\right) E\left(r_{t-1}^{2}\right)+c_{2} E\left(r_{t-2}^{2}\right)+2 c_{2} E\left(r_{t-1} r_{t-2}\right) .
\end{aligned}
$$

Devido à independência dos $\varepsilon_{t}$ temos que $E\left(r_{t-1} r_{t-2}\right)=0$ e, então,

$$
E\left(r_{t}^{2}\right)=c_{0}+\left(c_{1}+c_{2}\right) E\left(r_{t-1}^{2}\right)+c_{2} E\left(r_{t-2}^{2}\right)
$$

Usando (3.9) temos 


$$
E\left(r_{t}^{2}\right)=\frac{c_{0}}{1-\left(c_{1}+2 c_{2}\right)}
$$

Portanto devemos ter: $c_{1}+2 c_{2}<1$.

\section{GHARCH $(2,1)$}

$$
\begin{aligned}
& r_{t}=\sigma_{t} \varepsilon_{t}, \\
& \sigma_{t}^{2}=c_{0}+c_{1} r_{t-1}^{2}+c_{2}\left(r_{t-1}+r_{t-2}\right)^{2}+b_{1} \sigma_{t-1}^{2},
\end{aligned}
$$

$\varepsilon_{t} \sim$ i.i.d. $(0,1)$.

Se $r_{t}$ é estacionário temos que (3.9) vale.

Então,

$$
\begin{aligned}
E\left(r_{t}^{2}\right) & =E\left(\sigma_{t}^{2} \varepsilon_{t}^{2}\right)=E\left(\sigma_{t}^{2}\right) E\left(\varepsilon_{t}^{2}\right) \\
& =E\left(\sigma_{t}^{2}\right)=E\left(c_{0}+c_{1} r_{t-1}^{2}+c_{2}\left(r_{t-1}+r_{t-2}\right)^{2}+b_{1} \sigma_{t-1}^{2}\right) \\
& =c_{0}+\left(c_{1}+c_{2}\right) E\left(r_{t-1}^{2}\right)+c_{2} E\left(r_{t-2}^{2}\right)+2 c_{2} E\left(r_{t-1} r_{t-2}\right)+b_{1} E\left(\sigma_{t-1}^{2}\right) .
\end{aligned}
$$

Devido à independência dos $\varepsilon_{t}$ temos que $E\left(r_{t-1} r_{t-2}\right)=0$ e, então,

$$
E\left(r_{t}^{2}\right)=c_{0}+\left(c_{1}+c_{2}\right) E\left(r_{t-1}^{2}\right)+c_{2} E\left(r_{t-2}^{2}\right)+b_{1} E\left(\sigma_{t-1}^{2}\right) .
$$

Usando (3.9) temos

$$
E\left(r_{t}^{2}\right)=\frac{c_{0}}{1-\left(c_{1}+2 c_{2}+b_{1}\right)}
$$

e portanto $c_{1}+2 c_{2}+b_{1}<1$. 


\section{$\operatorname{GHARCH}(n, p)$}

$$
\begin{aligned}
& r_{t}=\sigma_{t} \varepsilon_{t}, \\
& \sigma_{t}^{2}=c_{0}+c_{1} r_{t-1}^{2}+\ldots+c_{n}\left(r_{t-1}+\ldots+r_{t-n}\right)^{2}+b_{1} \sigma_{t-1}^{2}+\ldots+b_{p} \sigma_{t-p}^{2},
\end{aligned}
$$

$\varepsilon_{t} \sim$ i.i.d. $(0,1)$.

Se $r_{t}$ é estacionário temos que

$$
\begin{aligned}
E\left(r_{t}^{2}\right) & =E\left(\sigma_{t}^{2} \varepsilon_{t}^{2}\right)=E\left(\sigma_{t}^{2}\right) E\left(\varepsilon_{t}^{2}\right)=E\left(\sigma_{t}^{2}\right) \\
& =E\left(c_{0}+c_{1} r_{t-1}^{2}+\ldots+c_{n}\left(r_{t-1}+\ldots+r_{t-n}\right)^{2}+b_{1} \sigma_{t-1}^{2}+\ldots+b_{p} \sigma_{t-p}^{2}\right) \\
& =c_{0}+\sum_{i=1}^{n} c_{i} E\left(\left(\sum_{j=1}^{i} r_{t-j}\right)^{2}\right)+\sum_{k=1}^{p} b_{k} E\left(\sigma_{t-k}^{2}\right) .
\end{aligned}
$$

Devido à independência dos $\varepsilon_{t}$ temos que $E\left(r_{t-i} r_{t-j}\right)=0, \forall i \neq j$ e, então,

$$
E\left(r_{t}^{2}\right)=c_{0}+\sum_{i=1}^{n} c_{i} E\left(\sum_{j=1}^{i} r_{t-j}^{2}\right)+\sum_{k=1}^{p} b_{k} E\left(\sigma_{t-k}^{2}\right),
$$

e usando (3.9) temos:

$$
E\left(r_{t}^{2}\right)=\frac{c_{0}}{1-\left(\sum_{i=1}^{n} i c_{i}+\sum_{k=1}^{p} b_{k}\right)}
$$

Portanto devemos ter $\sum_{i=1}^{n} i c_{i}+\sum_{k=1}^{p} b_{k}<1$. 
$\operatorname{GHARCH}(\boldsymbol{n}, \boldsymbol{p})$ parcimonioso com $n=a^{m}$.

$$
\begin{aligned}
r_{t} & =\sigma_{t} \varepsilon_{t}, \\
\sigma_{t}^{2} & =C_{0}+C_{1} r_{t-1}^{2}+C_{2}\left(r_{t-1}+\ldots+r_{t-a}\right)^{2}+\ldots \\
& +C_{m+1}\left(r_{t-1}+\ldots+r_{t-a^{m}}\right)^{2}+b_{1} \sigma_{t-1}^{2}+\ldots+b_{p} \sigma_{t-p}^{2},
\end{aligned}
$$

ou seja,

$$
\begin{aligned}
& r_{t}=\sigma_{t} \varepsilon_{t}, \\
& \sigma_{t}^{2}=C_{0}+\sum_{i=1}^{m+1} C_{i}\left(\sum_{j=1}^{a^{i-1}} r_{t-j}\right)^{2}+\sum_{k=1}^{p} b_{k} \sigma_{t-k}^{2},
\end{aligned}
$$

$\varepsilon_{t} \sim$ i.i.d. $(0,1)$.

Se $r_{t}$ é estacionário temos

$$
\begin{aligned}
\mathrm{E}\left(r_{t}^{2}\right) & =\mathrm{E}\left(\sigma_{t}^{2} \varepsilon_{t}^{2}\right)=\mathrm{E}\left(\sigma_{t}^{2}\right) \mathrm{E}\left(\varepsilon_{t}^{2}\right)=\mathrm{E}\left(\sigma_{t}^{2}\right) \\
& =\mathrm{E}\left(C_{0}+\sum_{i=1}^{m+1} C_{i}\left(\sum_{j=1}^{a^{i-1}} r_{t-j}\right)^{2}+\sum_{k=1}^{p} b_{k} \sigma_{t-k}^{2}\right) \\
& =C_{0}+\sum_{i=1}^{m+1} C_{i} \mathrm{E}\left[\left(\sum_{j=1}^{a^{i-1}} r_{t-j}\right)^{2}\right]+\sum_{k=1}^{p} b_{k} \mathrm{E}\left(\sigma_{t-k}^{2}\right) .
\end{aligned}
$$

Devido à independência dos $\varepsilon_{t}$ temos que $E\left(r_{t-i} r_{t-j}\right)=0, \forall i \neq j$ e, então,

$$
\mathrm{E}\left(r_{t}^{2}\right)=C_{0}+\sum_{i=1}^{m+1} C_{i} \sum_{j=1}^{a^{i-1}} \mathrm{E}\left(r_{t-j}^{2}\right)+\sum_{k=1}^{p} b_{k} \mathrm{E}\left(\sigma_{t-k}^{2}\right) .
$$

Usando (3.9) temos: 


$$
E\left(r_{t}^{2}\right)=\frac{C_{0}}{1-\left(\sum_{i=1}^{m+1} a^{i-1} C_{i}+\sum_{k=1}^{p} b_{k}\right)},
$$

do que decorre $\sum_{i=1}^{m+1} a^{i-1} C_{i}+\sum_{k=1}^{p} b_{k}<1$.

Note-se que o cálculo de $E\left(r_{t}^{2}\right)$ nos dá condições necessárias sobre os parâmetros, mas não suficientes.

Para obter condições suficientes temos que reformular o problema em termos de Cadeias de Markov. No entanto, não usaremos o caminho seguido por Engle (1982) e Bollerslev (1986) para determinar as condições suficientes de estacionariedade dos processos GARCH, devido ao fato que nos processos HARCH, a agrupação dos retornos envolve o termo $r_{t-i} r_{t-j}$, o que dificulta uma formulação similar.

Usaremos, então, o mesmo caminho adotado por Dacorogna et al. (1996) e estenderemos os resultados aí encontrados.

Para isso usaremos alguns resultados sobre cadeias de Markov dados por Meyn e Tweedie (1996), sumarizados no Capítulo 1.

Agora consideremos os processos HARCH como cadeias de Markov da seguinte forma.

A cadeia $X_{t}=\left(r_{t}, r_{t-1}\right)$, onde os valores $r_{t}$ e $r_{t-1}$ são dados pela equação (3.8), descreve um processo HARCH (2).

A cadeia $X_{t}=\left(r_{t-1}, \sigma_{t}\right)$, onde os valores $r_{t-1}$ e $\sigma_{t}$ são dados pela equação (3.11), descreve um processo $\operatorname{GHARCH}(2,1)$.

A cadeia $X_{t}=\left(r_{t-1}, \ldots, r_{t-n+1}, \sigma_{t}, \ldots, \sigma_{t-p+1}\right)$, onde os valores $r_{t-1}, \ldots, r_{t-n+1}$ e $\sigma_{t}, \ldots, \sigma_{t-p+1}$ são dados pela equação (3.13), descreve um processo GHARCH (n,p).

A cadeia $X_{t}=\left(r_{t-1}, \ldots, r_{t-a^{m}+1}, \sigma_{t}, \ldots, \sigma_{t-p+1}\right)$, onde os valores $r_{t-1}, \ldots, r_{t-a^{m}+1} \mathrm{e}$ $\sigma_{t}, \ldots, \sigma_{t-p+1}$ são dados pela equação (3.16), descreve um processo parcimonioso GHARCH (n,p) com $n=a^{m}$.

\section{Proposição 3.1.}

As cadeias de Markov que representam os processos HARCH (2), GHARCH $(2,1)$, GHARCH (n,p) e parcimonioso GHARCH (n,p) com $n=a^{m}$ são também cadeias fracas de Feller. 
Prova.

Seja $F_{y}(\cdot)$ a distribuição dos eventos $y$. Se temos que $y_{k} \rightarrow y$, então temos que mostrar que $F_{y_{k}} \rightarrow F_{y}$. Devido ao fato que as cadeias de Markov que descrevem os processos HARCH (2), GHARCH $(2,1)$, GHARCH $(n, p)$ e parcimonioso GHARCH $(n, p)$ com $n=a^{m}$ são expressas através de funções contínuas, isto é, para $\varepsilon_{t}$ fixo, a função $G, X_{t}=G\left(X_{t-1}, \varepsilon_{t}\right)$, é uma função contínua, então usando o teorema da convergência dominada temos o resultado.

\section{Proposição 3.2.}

As cadeias de Markov que representam os processos HARCH (2), GHARCH $(2,1)$, $\operatorname{GHARCH}(n, p)$ e parcimonioso $\operatorname{GHARCH}(n, p) \operatorname{com} n=a^{m}$ são também $T$-cadeias.

Prova.

Os processos HARCH (2), GHARCH $(2,1)$, GHARCH $(n, p)$ e parcimonioso GHARCH $(n, p)$, com $n=a^{m}$, são processos $\psi$-irredutíveis, e usando o resultado anterior, isto é, que estes processos são cadeias fracas de Feller temos o resultado.

\section{Proposição 3.3.}

As cadeias de Markov que representam os processos HARCH (2), GHARCH $(2,1)$, $\operatorname{GHARCH}(n, p)$ e parcimonioso $\operatorname{GHARCH}(n, p)$, com $n=a^{m}$, são recorrentes e cada uma delas possui medida invariante, para as condições abaixo indicadas.

\section{Prova.}

\section{$\underline{\text { HARCH (2) }}$}

A cadeia $X_{t}=\left(r_{t}, r_{t-1}\right)$, onde os valores $r_{t}$ e $r_{t-1}$ são dados pela equação (3.8), descreve um processo HARCH (2).

Sejam $c_{1}$ e $c_{2}$, coeficientes que satisfazem a condição: i) $c_{1}+2 c_{2}<1$, e escolhemos $\alpha$ tal que ii) $\alpha>c_{2}$ e iii) $c_{1}+c_{2}+\alpha<1$.

Definimos a função $V$ como $V(y, x):=\alpha x^{2}+y^{2}+2 c_{2} x y$.

Temos que 


$$
V(y, x)=\alpha x^{2}+y^{2}+2 c_{2} x y \geq c_{2} x^{2}+c_{2} y^{2}+2 c_{2} x y=\left(\sqrt{c_{2}} x+\sqrt{c_{2}} y\right)^{2} \geq 0 .
$$

Então temos que:

$$
\begin{aligned}
V\left(X_{t}\right)-E\left[V\left(X_{t+1}\right) \mid X_{t}\right]= & V\left(r_{t}, r_{t-1}\right)-E\left[V\left(r_{t+1}, r_{t}\right) \mid r_{t}, r_{t-1}\right] \\
= & \alpha r_{t-1}^{2}+r_{t}^{2}+2 c_{2} r_{t-1} r_{t}-E\left[\alpha r_{t}^{2}+r_{t+1}^{2}+2 c_{2} r_{t} r_{t+1} \mid r_{t}, r_{t-1}\right] \\
= & \alpha r_{t-1}^{2}+r_{t}^{2}+2 c_{2} r_{t-1} r_{t}-\alpha r_{t}^{2}- \\
& -\left(c_{0}+c_{1} r_{t}^{2}+c_{2}\left(r_{t}+r_{t-1}\right)^{2}\right) E\left[\varepsilon_{t+1}^{2}\right] \\
& -2 c_{2} E\left[\varepsilon_{t+1}\right]\left(c_{0}+c_{1} r_{t}^{2}+c_{2}\left(r_{t}+r_{t-1}\right)^{2}\right)^{1 / 2} r_{t} .
\end{aligned}
$$

Como $E\left(\varepsilon_{t}\right)=0$ e $E\left(\varepsilon_{t}^{2}\right)=1$, então temos:

$$
\begin{aligned}
V\left(X_{t}\right)-E\left[V\left(X_{t+1}\right) \mid X_{t}\right] & =\alpha r_{t-1}^{2}+r_{t}^{2}+2 c_{2} r_{t-1} r_{t}-\alpha r_{t}^{2}-\left(c_{0}+c_{1} r_{t}^{2}+c_{2}\left(r_{t}+r_{t-1}\right)^{2}\right) \\
& =\left(\alpha-c_{2}\right) r_{t-1}^{2}+\left(1-\alpha-c_{1}-c_{2}\right) r_{t}^{2}-c_{0} .
\end{aligned}
$$

Como $\left(\alpha-c_{2}\right)$ e $\left(1-\alpha-c_{1}-c_{2}\right)$ são positivos, a última expressão é tão grande quanto quisermos, se $\left(r_{t}, r_{t-1}\right)$ estão fora de um conjunto compacto.

Portanto, a cadeia $X_{t}=\left(r_{t}, r_{t-1}\right)$ que descreve um processo HARCH (2) é recorrente e tem uma medida invariante.

\section{$\underline{\operatorname{GHARCH}(2,1)}$}

A cadeia $X_{t}=\left(\sigma_{t}, r_{t-1}\right)$, onde os valores $r_{t-1}$ e $\sigma_{t}$ são dados pela equação (3.11), descreve um processo GHARCH $(2,1)$.

Sejam $c_{1}, c_{2}$ e $b_{1}$, coeficientes que satisfazem a condição: i) $c_{1}+2 c_{2}+b_{1}<1$, e escolhemos $\alpha$ tal que ii) $\alpha>c_{2}$ e iii) $c_{1}+c_{2}+b_{1}+\alpha<1$.

Definimos a função $V$ como $V(y, x):=\alpha x^{2}+y^{2}$

Então, $V(y, x)=\alpha x^{2}+y^{2} \geq 0$,

e temos 


$$
\begin{aligned}
V\left(X_{t}\right)-E\left[V\left(X_{t+1}\right) \mid X_{t}\right]= & V\left(\sigma_{t}, r_{t-1}\right)-E\left[V\left(\sigma_{t+1}, r_{t}\right) \mid \sigma_{t}, r_{t-1}\right] \\
= & \alpha r_{t-1}^{2}+\sigma_{t}^{2}-E\left[\sigma_{t+1}^{2}+\alpha \sigma_{t}^{2} \varepsilon_{t}^{2} \mid \sigma_{t}, r_{t-1}\right] \\
= & \alpha r_{t-1}^{2}+\sigma_{t}^{2}-\alpha \sigma_{t}^{2} E\left[\varepsilon_{t}^{2}\right]- \\
& -E\left[c_{0}+c_{1} r_{t}^{2}+c_{2}\left(r_{t}+r_{t-1}\right)^{2}+b_{1} \sigma_{t}^{2} \mid \sigma_{t}, r_{t-1}\right] \\
= & \alpha r_{t-1}^{2}+\sigma_{t}^{2}-\alpha \sigma_{t}^{2} E\left[\varepsilon_{t}^{2}\right]- \\
& -E\left[c_{0}+c_{1} \sigma_{t}^{2} \varepsilon_{t}^{2}+c_{2}\left(\sigma_{t} \varepsilon_{t}+r_{t-1}\right)^{2}+b_{1} \sigma_{t}^{2} \mid \sigma_{t}, r_{t-1}\right] \\
= & \alpha r_{t-1}^{2}+\sigma_{t}^{2}-c_{0}-c_{1} \sigma_{t}^{2} E\left[\varepsilon_{t}^{2}\right]-c_{2} \sigma_{t}^{2} E\left[\varepsilon_{t}^{2}\right]- \\
& -c_{2} r_{t-1}^{2}-2 c_{2} \sigma_{t} r_{t-1} E\left[\varepsilon_{t}\right]-b_{1} \sigma_{t}^{2}-\alpha \sigma_{t}^{2} E\left[\varepsilon_{t}^{2}\right] .
\end{aligned}
$$

Como $E\left(\varepsilon_{t}\right)=0$ e $E\left(\varepsilon_{t}^{2}\right)=1$, então temos:

$$
\begin{aligned}
V\left(X_{t}\right)-E\left[V\left(X_{t+1}\right) \mid X_{t}\right] & =\alpha r_{t-1}^{2}+\sigma_{t}^{2}-c_{0}-c_{1} \sigma_{t}^{2}-c_{2} \sigma_{t}^{2}-c_{2} r_{t-1}^{2}-b_{1} \sigma_{t}^{2} \\
& =\left(\alpha-c_{2}\right) r_{t-1}^{2}+\left(1-\alpha-c_{1}-c_{2}-b_{1}\right) \sigma_{t}^{2}-c_{0}
\end{aligned}
$$

Novamente, como $\left(\alpha-c_{2}\right)$ e $\left(1-\alpha-c_{1}-c_{2}\right)$ são positivos, a última expressão é tão grande quanto quisermos, se $\left(\sigma_{t}, r_{t-1}\right)$ estão fora de um conjunto compacto.

Portanto, a cadeia $X_{t}=\left(\sigma_{t}, r_{t-1}\right)$ que descreve um processo $\operatorname{GHARCH}(2,1)$ é recorrente e tem uma medida invariante.

\section{$\underline{\operatorname{GHARCH}(n, p)}$}

A cadeia $X_{t}=\left(r_{t-1}, \ldots, r_{t-n+1}, \sigma_{t}, \ldots, \sigma_{t-p+1}\right)$, onde os valores $r_{t-1}, \ldots, r_{t-n+1}$ e $\sigma_{t}, \ldots, \sigma_{t-p+1}$ são dados pela equação (3.13), descreve um processo $\operatorname{GHARCH}(n, p)$. Definimos a função $V$, como:

$$
V\left(X_{t}\right)=V\left(r_{t-1}, \ldots, r_{t-n+1}, \sigma_{t}, \ldots, \sigma_{t-p+1}\right):=\sum_{i=1}^{n-1} \alpha_{i} r_{t-i}^{2}+2 \sum_{i=1}^{n-2} \sum_{j=i+1}^{n-1} \beta_{j} r_{t-i} r_{t-j}+\sum_{i=0}^{p-1} \gamma_{i} \sigma_{t-i}^{2}
$$

Agora vamos calcular $V\left(X_{t}\right)-E\left[V\left(X_{t+1}\right) \mid X_{t}\right]$, mas vamos fazê-lo por partes, assim: 


$$
\begin{aligned}
& E\left[\sum_{i=1}^{n-1} \alpha_{i} r_{t+1-i}^{2} \mid X_{t}\right]=E\left[\alpha_{1} r_{t}^{2} \mid X_{t}\right]+\sum_{i=1}^{n-2} \alpha_{i+1} r_{t-i}^{2}=\alpha_{1} \sigma_{t}^{2}+\sum_{i=1}^{n-2} \alpha_{i+1} r_{t-i}^{2} . \\
& E\left[2 \sum_{i=1}^{n-2} \sum_{j=i+1}^{n-1} \beta_{j} r_{t+1-i} r_{t+1-j} \mid X_{t}\right]=E\left[2 \sum_{i=0}^{n-3} \sum_{j=i+1}^{n-2} \beta_{j+1} r_{t-i} r_{t-j} \mid X_{t}\right] \\
& =E\left[2 r_{t} \sum_{j=1}^{n-2} \beta_{j+1} r_{t-j} \mid X_{t}\right]+2 \sum_{i=1}^{n-3} \sum_{j=i+1}^{n-2} \beta_{j+1} r_{t-i} r_{t-j} \\
& =2 \sum_{i=1}^{n-3} \sum_{j=i+1}^{n-2} \beta_{j+1} r_{t-i} r_{t-j} \text {. } \\
& E\left[\sum_{i=0}^{p-1} \gamma_{i} \sigma_{t+1-i}^{2} \mid X_{t}\right]=E\left[\gamma_{0} \sigma_{t+1}^{2} \mid X_{t}\right]+\sum_{i=1}^{p-1} \gamma_{i} \sigma_{t+1-i}^{2}, \\
& E\left[\sum_{i=0}^{p-1} \gamma_{i} \sigma_{t+1-i}^{2} \mid X_{t}\right]=E\left[\gamma_{0}\left(c_{0}+c_{1} r_{t}^{2}+\ldots+c_{n}\left(r_{t}+\ldots+r_{t-n+1}\right)^{2}\right) \mid X_{t}\right]+ \\
& +E\left[\gamma_{0}\left(b_{1} \sigma_{t}^{2}+\ldots+b_{p} \sigma_{t-p+1}^{2}\right) \mid X_{t}\right]+\sum_{i=0}^{p-2} \gamma_{i+1} \sigma_{t-i}^{2}, \\
& E\left[\sum_{i=0}^{p-1} \gamma_{i} \sigma_{t+1-i}^{2} \mid X_{t}\right]=E\left[\gamma_{0}\left(c_{0}+r_{t}^{2} \sum_{i=1}^{n} c_{i}+2 r_{t} \sum_{i=1}^{n-1} r_{t-i} \sum_{j=i+1}^{n} c_{j}\right) \mid X_{t}\right]+ \\
& +E\left[\gamma_{0}\left(\sum_{i=1}^{n-1} r_{t-i}^{2} \sum_{j=i+1}^{n} c_{j}\right) \mid X_{t}\right]+ \\
& +E\left[\gamma_{0}\left(2 \sum_{i=1}^{n-2} \sum_{j=i+1}^{n-1} r_{t-i} r_{t-j} \sum_{k=j+1}^{n} c_{k}\right) \mid X_{t}\right]+ \\
& +\gamma_{0} \sum_{i=0}^{p-1} b_{i+1} \sigma_{t-i}^{2}+\sum_{i=0}^{p-2} \gamma_{i+1} \sigma_{t-i}^{2} \\
& =\gamma_{0}\left(c_{0}+\sigma_{t}^{2} \sum_{i=1}^{n} c_{i}+\sum_{i=1}^{n-1} r_{t-i}^{2} \sum_{j=i+1}^{n} c_{j}+2 \sum_{i=1}^{n-2} \sum_{j=i+1}^{n-1} r_{t-i} r_{t-j} \sum_{k=j+1}^{n} c_{k}\right)+ \\
& +\gamma_{0} \sum_{i=0}^{p-1} b_{i+1} \sigma_{t-i}^{2}+\sum_{i=0}^{p-2} \gamma_{i+1} \sigma_{t-i}^{2} \text {. }
\end{aligned}
$$


Portanto, temos que:

$$
\begin{aligned}
V\left(X_{t}\right)-E\left[V\left(X_{t+1}\right) \mid X_{t}\right]= & \sum_{i=1}^{n-1} \alpha_{i} r_{t-i}^{2}+2 \sum_{i=1}^{n-2} \sum_{j=i+1}^{n-1} \beta_{j} r_{t-i} r_{t-j}+\sum_{i=0}^{p-1} \gamma_{i} \sigma_{t-i}^{2}-\alpha_{1} \sigma_{t}^{2}- \\
& -\sum_{i=1}^{n-2} \alpha_{i+1} r_{t-i}^{2}-2 \sum_{i=1}^{n-3} \sum_{j=i+1}^{n-2} \beta_{j+1} r_{t-i} r_{t-j}- \\
& -\gamma_{0}\left(c_{0}+\sigma_{t}^{2} \sum_{i=1}^{n} c_{i}+\sum_{i=1}^{n-1} r_{t-i}^{2} \sum_{j=i+1}^{n} c_{j}\right)- \\
& -2 \gamma_{0} \sum_{i=1}^{n-2} \sum_{j=i+1}^{n-1} r_{t-i} r_{t-j} \sum_{k=j+1}^{n} c_{k}-\gamma_{0} \sum_{i=0}^{p-1} b_{i+1} \sigma_{t-i}^{2}-\sum_{i=0}^{p-2} \gamma_{i+1} \sigma_{t-i}^{2},
\end{aligned}
$$

e agrupando temos:

$$
\begin{aligned}
V\left(X_{t}\right)-E\left[V\left(X_{t+1}\right) \mid X_{t}\right] & =\sum_{i=1}^{n-2}\left(\alpha_{i}-\alpha_{i+1}-\gamma_{0} \sum_{j=i+1}^{n} c_{j}\right) r_{t-i}^{2}+\left(\alpha_{n-1}-\gamma_{0} c_{n}\right) r_{t-n+1}^{2}+ \\
& +2 \sum_{i=1}^{n-3} \sum_{j=i+1}^{n-2}\left(\beta_{j}-\beta_{j+1}-\gamma_{0} \sum_{k=j+1}^{n} c_{k}\right) r_{t-i} r_{t-j}+ \\
& +2 \sum_{i=1}^{n-2}\left(\beta_{n-1}-\gamma_{0} c_{n}\right) r_{t-i} r_{t-n+1}+ \\
& +\sum_{i=1}^{p-2}\left(\gamma_{i}-\gamma_{i+1}-\gamma_{0} b_{i+1}\right) \sigma_{t-i}^{2}+\left(\gamma_{p-1}-\gamma_{0} b_{p}\right) \sigma_{t-p+1}^{2}+ \\
& +\left(\gamma_{0}-\gamma_{1}-\gamma_{0} b_{1}-\alpha_{1}-\gamma_{0} \sum_{i=1}^{n} c_{i}\right) \sigma_{t}^{2}-\gamma_{0} c_{0}
\end{aligned}
$$

Então podemos escolher os $\beta_{j}$ como segue:

$$
\beta_{j}=\gamma_{0} \sum_{i=j+1}^{n} \sum_{k=i}^{n} c_{k}=\gamma_{0}\left(\sum_{k=j+1}^{n} c_{k}+\sum_{k=j+2}^{n} c_{k}+\ldots+\sum_{k=n-1}^{n} c_{k}+c_{n}\right), \forall j \in\{2, \ldots, n-1\},
$$

portanto, temos que:

$$
\beta_{j}=\beta_{j+1}+\gamma_{0} \sum_{k=j+1}^{n} c_{k}, \forall j \in\{2, \ldots, n-2\} \text { e } \beta_{n-1}=\gamma_{0} c_{n} .
$$


Então vamos ter a eliminação dos produtos duplos em (3.18) e, portanto,

$$
\begin{aligned}
V\left(X_{t}\right)-E\left[V\left(X_{t+1}\right) \mid X_{t}\right] & =\sum_{i=1}^{n-2}\left(\alpha_{i}-\alpha_{i+1}-\gamma_{0} \sum_{j=i+1}^{n} c_{j}\right) r_{t-i}^{2}+ \\
& +\left(\alpha_{n-1}-\gamma_{0} c_{n}\right) r_{t-n+1}^{2}+ \\
& +\sum_{i=1}^{p-2}\left(\gamma_{i}-\gamma_{i+1}-\gamma_{0} b_{i+1}\right) \sigma_{t-i}^{2}+ \\
& +\left(\gamma_{p-1}-\gamma_{0} b_{p}\right) \sigma_{t-p+1}^{2}+ \\
& +\left(\gamma_{0}-\gamma_{1}-\gamma_{0} b_{1}-\alpha_{1}-\gamma_{0} \sum_{i=1}^{n} c_{i}\right) \sigma_{t}^{2}-\gamma_{0} c_{0} .
\end{aligned}
$$

Note que com os valores dos $\beta_{j}$ escolhidos, e se temos que os valores dos $\alpha_{i}$ são tais que $\alpha_{i}>\gamma_{0} \sum_{j=i+1}^{n} \sum_{k=j}^{n} c_{k}, \forall i \in\{1, \ldots, n-1\}$, então a função $V\left(X_{t}\right) \geq 0$. Para mostrar isto, basta considerar as variáveis aleatórias $Y_{2}, \ldots, Y_{n}, Z_{1}, \ldots, Z_{n-1}$ i.i.d. $N(0,1)$. Então o vetor aleatório dado pela combinação:

$$
W_{i}=\gamma_{0} \sum_{j=i+1}^{n}\left(\sum_{k=j}^{n} c_{k}\right)^{1 / 2} Y_{j}+\left(\alpha_{i}-\gamma_{0} \sum_{j=i+1}^{n} \sum_{k=j}^{n} c_{k}\right)^{1 / 2} Z_{i}, i=1, \ldots, n-1
$$

tem matriz de variância:

$$
\left(\begin{array}{ccccc}
\alpha_{1} & \beta_{2} & \beta_{3} & \ldots & \beta_{n-1} \\
\beta_{2} & \alpha_{2} & \beta_{3} & \ldots & \beta_{n-1} \\
\vdots & & \ddots & & \vdots \\
\beta_{n-1} & \beta_{n-1} & \beta_{n-1} & \ldots & \alpha_{n-1}
\end{array}\right)
$$

E portanto, como toda matriz de variâncias é definida não negativa, temos que

$$
V\left(X_{t}\right)=\sum_{i=1}^{n-1} \alpha_{i} r_{t-i}^{2}+2 \sum_{i=1}^{n-2} \sum_{j=i+1}^{n-1} \beta_{j} r_{t-i} r_{t-j}+\sum_{i=0}^{p-1} \gamma_{i} \sigma_{t-i}^{2} \geq \sum_{i=1}^{n-1} \alpha_{i} r_{t-i}^{2}+2 \sum_{i=1}^{n-2} \sum_{j=i+1}^{n-1} \beta_{j} r_{t-i} r_{t-j} \geq 0
$$

para valores de $\gamma_{i} \geq 0, \forall i=1, \ldots, p$. 
Agora vamos demonstrar que existem valores para $\alpha_{i}, \beta_{i}$ e $\gamma_{i}$, tais que $V\left(X_{n}\right)-E\left[V\left(X_{n+1}\right) \mid X_{n}\right] \geq 0, X_{n} \in C^{c}$, onde $C$ é um conjunto compacto.

Primeiro tomemos $\gamma_{0}=1$, então a equação (3.19) fica assim:

$$
\begin{aligned}
V\left(X_{t}\right)-E\left[V\left(X_{t+1}\right) \mid X_{t}\right] & =\sum_{i=1}^{n-2}\left(\alpha_{i}-\alpha_{i+1}-\sum_{j=i+1}^{n} c_{j}\right) r_{t-i}^{2}+\left(\alpha_{n-1}-c_{n}\right) r_{t-n+1}^{2}+ \\
& +\sum_{i=1}^{p-2}\left(\gamma_{i}-\gamma_{i+1}-b_{i+1}\right) \sigma_{t-i}^{2}+\left(\gamma_{p-1}-b_{p}\right) \sigma_{t-p+1}^{2}+ \\
& +\left(1-\gamma_{1}-b_{1}-\alpha_{1}-\sum_{i=1}^{n} c_{i}\right) \sigma_{t}^{2}-c_{0}
\end{aligned}
$$

Agora consideramos:

$$
\sum_{i=1}^{n} i c_{i}+\sum_{k=1}^{p} b_{k}=\sum_{i=1}^{n} \sum_{j=i}^{n} c_{i}+\sum_{k=1}^{p} b_{k}<1 .
$$

Note-se que temos que:

$$
\alpha_{i}>\sum_{j=i+1}^{n} \sum_{k=j}^{n} c_{k}, \text { para } i=2, \ldots, n-1
$$

Portanto as seguintes desigualdades são validas:

$$
\begin{gathered}
\alpha_{i}>\sum_{j=i+2}^{n} \sum_{k=j}^{n} c_{k}+\sum_{k=i+1}^{n} c_{k}, \\
\alpha_{i}-\sum_{k=i+1}^{n} c_{k}>\sum_{j=i+2}^{n} \sum_{k=j}^{n} c_{k} .
\end{gathered}
$$

Como a desigualdade (3.23) é estrita, então podemos escolher $\alpha_{i+1}$ satisfazendo (3.22) tal que:

$$
\alpha_{i}-\sum_{k=i+1}^{n} c_{k}>\alpha_{i+1}>\sum_{j=i+2}^{n} \sum_{k=j}^{n} c_{k} .
$$


De maneira similar para $\gamma_{i}$ temos que:

$$
\gamma_{i}>\sum_{j=i+1}^{p} b_{j}, \text { para } i=2, \ldots, p-1 .
$$

Portanto as seguintes desigualdades são validas:

$$
\begin{gathered}
\gamma_{i}>\sum_{j=i+2}^{p} b_{j}+b_{i+1}, \\
\gamma_{i}-b_{i+1}>\sum_{j=i+2}^{p} b_{j} .
\end{gathered}
$$

Como a desigualdade (3.26) é estrita, então podemos escolher $\gamma_{i+1}$ satisfazendo (3.25) tal que:

$$
\gamma_{i}-b_{i+1}>\gamma_{i+1}>\sum_{j=i+2}^{n} b_{j}
$$

Agora faltam escolher os valores de $\alpha_{1}$ e $\gamma_{1}$, para isso usamos (3.21) assim:

$$
\begin{aligned}
& \sum_{i=1}^{n} \sum_{j=i}^{n} c_{i}+\sum_{k=1}^{p} b_{k}<1 \Rightarrow 1-\sum_{i=1}^{n} \sum_{j=i}^{n} c_{i}-\sum_{k=1}^{p} b_{k}>0 \\
& \Rightarrow 1-\sum_{i=2}^{n} \sum_{j=i}^{n} c_{i}-\sum_{k=2}^{p} b_{k}-\sum_{j=1}^{n} c_{i}-b_{1}>0,
\end{aligned}
$$

e portanto:

$$
1-\sum_{j=1}^{n} c_{i}-b_{1}>\sum_{i=2}^{n} \sum_{j=i}^{n} c_{i}+\sum_{k=2}^{p} b_{k} .
$$

Como a desigualdade (3.28) é estrita, então podemos escolher $\gamma_{1}$ e $\alpha_{1}$ satisfazendo (3.22) e (3.25), tal que:

$$
1-\sum_{j=1}^{n} c_{i}-b_{1}>\gamma_{1}+\alpha_{1}>\sum_{i=2}^{n} \sum_{j=i}^{n} c_{i}+\sum_{k=2}^{p} b_{k} .
$$


Segue das relações (3.29), (3.27) e (3.24) que $\left(\alpha_{i}-\alpha_{i+1}-\sum_{j=i+1}^{n} c_{j}\right)>0$ para $i=1, \ldots, n-2, \quad\left(\alpha_{n-1}-c_{n}\right)>0, \quad\left(\gamma_{i}-\gamma_{i+1}-b_{i+1}\right)>0 \quad$ para $\quad i=1, \ldots, p-2$, $\left(\gamma_{p-1}-b_{p}\right)>0$ e $\left(1-\gamma_{1}-b_{1}-\alpha_{1}-\sum_{i=1}^{n} c_{i}\right)>0$.

Então $V\left(X_{t}\right)-E\left[V\left(X_{t+1}\right) \mid X_{t}\right]$ pode ser tão grande quanto quisermos se $X_{t}$ está fora de um conjunto compacto.

Portanto a cadeia $X_{t}=\left(r_{t-1}, \ldots, r_{t-n+1}, \sigma_{t}, \ldots, \sigma_{t-p+1}\right)$ que descreve um processo $\operatorname{GHARCH}(n, p)$ é recorrente e tem uma medida invariante.

\section{$\underline{\operatorname{GHARCH}(\boldsymbol{n}, \boldsymbol{p}) \text { parcimonioso com } n=a^{m}}$}

A cadeia $X_{t}=\left(r_{t-1}, \ldots, r_{t-a^{m}+1}, \sigma_{t}, \ldots, \sigma_{t-p+1}\right)$, onde os valores $r_{t-1}, \ldots, r_{t-a^{m}+1} \mathrm{e}$ $\sigma_{t}, \ldots, \sigma_{t-p+1}$ são dados pela equação (3.16), descreve um processo parcimonioso $\operatorname{GHARCH}(n, p) \operatorname{com} n=a^{m}$.

Definimos a função $V$, como:

$$
V\left(X_{t}\right)=V\left(r_{t-1}, \ldots, r_{t-a^{m}+1}, \sigma_{t}, \ldots, \sigma_{t-p+1}\right):=\sum_{i=1}^{a^{m}-1} \alpha_{i} r_{t-i}^{2}+2 \sum_{i=1}^{a^{m}-2} \sum_{j=i+1}^{a^{m}-1} \beta_{j} r_{t-i} r_{t-j}+\sum_{i=0}^{p-1} \gamma_{i} \sigma_{t-i}^{2}
$$

Agora vamos calcular $V\left(X_{t}\right)-E\left[V\left(X_{t+1}\right) \mid X_{t}\right]$, mas vamos fazê-lo por partes, assim:

$$
\begin{gathered}
E\left[\sum_{i=1}^{a^{m}-1} \alpha_{i} r_{t+1-i}^{2} \mid X_{t}\right]=E\left[\sum_{i=0}^{a^{m}-2} \alpha_{i+1} r_{t-i}^{2} \mid X_{t}\right]=E\left[\alpha_{1} r_{t}^{2} \mid X_{t}\right]+\sum_{i=1}^{a^{m}-2} \alpha_{i+1} r_{t-i}^{2} \\
=\alpha_{1} \sigma_{t}^{2}+\sum_{i=1}^{a^{m}-2} \alpha_{i+1} r_{t-i}^{2}, \\
E\left[2 \sum_{i=1}^{a^{m}-2} \sum_{j=i+1}^{a^{m}-1} \beta_{j} r_{t+1-i} r_{t+1-j} \mid X_{t}\right]=E\left[2 \sum_{i=0}^{a^{m}-3} \sum_{j=i+1}^{a^{m}-2} \beta_{j+1} r_{t-i} r_{t-j} \mid X_{t}\right],
\end{gathered}
$$




$$
\begin{aligned}
& E\left[2 \sum_{i=1}^{a^{m}-2} \sum_{j=i+1}^{a^{m}-1} \beta_{j} r_{t+1-i} r_{t+1-j} \mid X_{t}\right]=E\left[2 r_{t} \sum_{j=1}^{a^{m}-2} \beta_{j+1} r_{t-j} \mid X_{t}\right]+2 \sum_{i=1}^{a^{m}-3} \sum_{j=i+1}^{a^{m}-2} \beta_{j+1} r_{t-i} r_{t-j} \\
& =2 \sum_{i=1}^{a^{m}-3} \sum_{j=i+1}^{a^{m}-2} \beta_{j+1} r_{t-i} r_{t-j} \text {, } \\
& E\left[\sum_{i=0}^{p-1} \gamma_{i} \sigma_{t+1-i}^{2} \mid X_{t}\right]=E\left[\gamma_{0} \sigma_{t+1}^{2} \mid X_{t}\right]+\sum_{i=1}^{p-1} \gamma_{i} \sigma_{t+1-i}^{2} \\
& =E\left[\gamma_{0}\left(C_{0}+C_{1} r_{t}^{2}+\ldots+C_{m+1}\left(r_{t}+\ldots+r_{t-a^{m}+1}\right)^{2}\right) \mid X_{t}\right]+ \\
& +E\left[\gamma_{0}\left(b_{1} \sigma_{t}^{2}+\ldots+b_{p} \sigma_{t-p+1}^{2}\right) \mid X_{t}\right]+\sum_{i=0}^{p-2} \gamma_{i+1} \sigma_{t-i}^{2}, \\
& E\left[\sum_{i=0}^{p-1} \gamma_{i} \sigma_{t+1-i}^{2} \mid X_{t}\right]=E\left[\gamma_{0}\left(C_{0}+r_{t}^{2} \sum_{i=1}^{m+1} C_{i}+2 r_{t} \sum_{l=0}^{m-1} \sum_{i=a^{l}}^{l+1} r_{t-i} \sum_{j=l+2}^{m+1} C_{j}\right) \mid X_{t}\right]+ \\
& +E\left[\gamma_{0}\left(\sum_{l=0}^{m-1} \sum_{i=a^{l}}^{a^{l+1}-1} r_{t-i}^{2} \sum_{j=l+2}^{m+1} C_{j}+2 \sum_{l=1}^{m-1} \sum_{i=a^{l}-1}^{a^{l+1}-2} \sum_{j=i+1}^{l+1} r_{t-i} r_{t-j} \sum_{k=l+2}^{m+1} C_{k}\right) \mid X_{t}\right]+ \\
& +E\left[\gamma_{0}\left(2 \sum_{i=1}^{a^{1}-2} \sum_{j=i+1}^{a^{1}-1} r_{t-i} r_{t-j} \sum_{k=2}^{m+1} C_{k}\right) \mid X_{t}\right]+\gamma_{0} \sum_{i=0}^{p-1} b_{i+1} \sigma_{t-i}^{2}+\sum_{i=0}^{p-2} \gamma_{i+1} \sigma_{t-i}^{2} \text {, } \\
& E\left[\sum_{i=0}^{p-1} \gamma_{i} \sigma_{t+1-i}^{2} \mid X_{t}\right]=\gamma_{0}\left(C_{0}+\sigma_{t}^{2} \sum_{i=1}^{m+1} C_{i}+\sum_{l=0}^{m-1} \sum_{i=a^{l}}^{a^{l+1}-1} r_{t-i}^{2} \sum_{j=l+2}^{m+1} C_{j}\right)+ \\
& +\gamma_{0}\left(2 \sum_{l=1}^{m-1} \sum_{i=a^{l}-1}^{l+1} \sum_{j=i+1}^{a^{l+1}-1} r_{t-i} r_{t-j} \sum_{k=l+2}^{m+1} C_{k}\right)+ \\
& +2 \gamma_{0} \sum_{i=1}^{a^{1}-2} \sum_{j=i+1}^{a^{1}-1} r_{t-i} r_{t-j} \sum_{k=2}^{m+1} C_{k}+\gamma_{0} \sum_{i=0}^{p-1} b_{i+1} \sigma_{t-i}^{2}+\sum_{i=0}^{p-2} \gamma_{i+1} \sigma_{t-i}^{2} .
\end{aligned}
$$

Portanto, temos que: 


$$
\begin{aligned}
V\left(X_{t}\right)-E\left[V\left(X_{t+1}\right) \mid X_{t}\right]= & \sum_{i=1}^{a^{m}-1} \alpha_{i} r_{t-i}^{2}+2 \sum_{i=1}^{a^{m}-2} \sum_{j=i+1}^{a^{m}-1} \beta_{j} r_{t-i} r_{t-j}+\sum_{i=0}^{p-1} \gamma_{i} \sigma_{t-i}^{2}-\alpha_{1} \sigma_{t}^{2}- \\
& -\sum_{i=1}^{a^{m}-2} \alpha_{i+1} r_{t-i}^{2}-2 \sum_{i=1}^{a^{m}-3} \sum_{j=i+1}^{a^{m}-2} \beta_{j+1} r_{t-i} r_{t-j}- \\
& -\gamma_{0}\left(C_{0}+\sigma_{t}^{2} \sum_{i=1}^{m+1} C_{i}+\sum_{l=0}^{m-1} \sum_{i=a^{l}}^{a^{l+1}-1} r_{t-i}^{2} \sum_{j=l+2}^{m+1} C_{j}\right)- \\
& -2 \gamma_{0} \sum_{l=1}^{m-1} \sum_{i=a^{l}-1}^{a^{l+1}-2} \sum_{j=i+1}^{l+1} r_{t-i} r_{t-j} \sum_{k=l+2}^{m+1} C_{k}- \\
& -2 \gamma_{0} \sum_{i=1}^{a^{1}-2} \sum_{j=i+1}^{a^{1}-1} r_{t-i} r_{t-j} \sum_{k=2}^{m+1} C_{k}-\gamma_{0} \sum_{i=0}^{p-1} b_{i+1} \sigma_{t-i}^{2}-\sum_{i=0}^{p-2} \gamma_{i+1} \sigma_{t-i}^{2} .
\end{aligned}
$$

Sejam $\alpha_{a^{m}}=\beta_{a^{m}}=\gamma_{p}=0$, e agrupando temos:

$$
\begin{aligned}
V\left(X_{t}\right)-E\left[V\left(X_{t+1}\right) \mid X_{t}\right] & =\sum_{l=0}^{m-1} \sum_{i=a^{l}}^{a^{l+1}-1}\left(\alpha_{i}-\alpha_{i+1}-\gamma_{0} \sum_{j=l+2}^{m+1} C_{j}\right) r_{t-i}^{2}+ \\
& +2 \sum_{l=1}^{m-1} \sum_{i=a^{l}-1}^{l+1} \sum_{j=i+1}^{a^{l+1}-1}\left(\beta_{j}-\beta_{j+1}-\gamma_{0} \sum_{k=l+2}^{m+1} C_{k}\right) r_{t-i} r_{t-j} \\
& +\sum_{i=1}^{a-2} \sum_{j=i+1}^{a-1}\left(\beta_{j}-\beta_{j+1}-\gamma_{0} \sum_{k=2}^{m+1} C_{k}\right) r_{t-i} r_{t-j}+ \\
& +\sum_{i=1}^{p-1}\left(\gamma_{i}-\gamma_{i+1}-\gamma_{0} b_{i+1}\right) \sigma_{t-i}^{2}+ \\
& +\left(\gamma_{0}-\gamma_{1}-\gamma_{0} b_{1}-\alpha_{1}-\gamma_{0} \sum_{i=1}^{m+1} C_{i}\right) \sigma_{t}^{2}-\gamma_{0} C_{0} .
\end{aligned}
$$

Seja $l$, tal que $j \in\left\{a^{l}, a^{l}+1, \ldots, a^{l+1}-1\right\}$, então podemos escolher os $\beta_{j}$ como segue:

$$
\beta_{j}=\gamma_{0} \sum_{k=j}^{a^{l+1}-1} \sum_{i=l+2}^{m+1} C_{i}+\gamma_{0} \sum_{s=l+1}^{m-1} \sum_{k=a^{s}}^{a^{s+1}-1} \sum_{i=s+2}^{m+1} C_{i}, \forall j \in\left\{2, \ldots, a^{m}-1\right\}
$$

portanto, temos que $\beta_{j}-\beta_{j+1}=\gamma_{0} \sum_{i=l+2}^{m+1} C_{i}$, com $l$, tal que $j \in\left\{a^{l}, a^{l}+1, \ldots, a^{l+1}-1\right\}$.

Então vamos ter a eliminação dos produtos duplos em (3.30) e, portanto, 


$$
\begin{aligned}
V\left(X_{t}\right)-E\left[V\left(X_{t+1}\right) \mid X_{t}\right] & =\sum_{l=0}^{m-1} \sum_{i=a^{l}}^{a^{l+1}-1}\left(\alpha_{i}-\alpha_{i+1}-\gamma_{0} \sum_{j=l+2}^{m+1} C_{j}\right) r_{t-i}^{2}+ \\
& +\sum_{i=1}^{p-1}\left(\gamma_{i}-\gamma_{i+1}-\gamma_{0} b_{i+1}\right) \sigma_{t-i}^{2}+ \\
& +\left(\gamma_{0}-\gamma_{1}-\gamma_{0} b_{1}-\alpha_{1}-\gamma_{0} \sum_{i=1}^{m+1} C_{i}\right) \sigma_{t}^{2}-\gamma_{0} C_{0} .
\end{aligned}
$$

Note que com os valores dos $\beta_{j}$ escolhidos, e se temos que os valores dos $\alpha_{j}$ são tais que $\alpha_{j}>\gamma_{0} \sum_{k=j}^{a^{l+1}-1} \sum_{i=l+2}^{m+1} C_{i}+\gamma_{0} \sum_{s=l+1}^{m-1} \sum_{k=a^{s}}^{a^{s+1}-1} \sum_{i=s+2}^{m+1} C_{i}, \quad \forall i \in\left\{1, \ldots, a^{m}-1\right\}$, então a função $V\left(X_{t}\right) \geq 0$. Para mostrar isto, basta considerar as variáveis aleatórias $Y_{1}, \ldots, Y_{a^{m}-1}, Z_{1}, \ldots, Z_{a^{m}-1}$ i.i.d. $N(0,1)$. Então o vetor aleatório dado pela combinação

$$
\begin{aligned}
W_{i} & =\gamma_{0} \sum_{k=j}^{a^{l+1}-1}\left(\sum_{i=l+2}^{m+1} C_{i}\right)^{1 / 2} Y_{j}+\sum_{s=l+1}^{m-1} \sum_{k=a^{s}}^{a^{s+1}}\left(\sum_{i=s+2}^{m+1} C_{i}\right)^{1 / 2} Y_{s}+ \\
& +\left(\alpha_{i}-\gamma_{0}\left(\sum_{k=j}^{a^{l+1}-1} \sum_{i=l+2}^{m+1} C_{i}+\sum_{s=l+1}^{m-1} \sum_{k=a^{s}}^{a^{s+1}} \sum_{i=s+2}^{m+1} C_{i}\right)\right)^{1 / 2} Z_{i},
\end{aligned}
$$

$i=1, \ldots, a^{m}-1$, tem matriz de variância:

$$
\left(\begin{array}{ccccc}
\alpha_{1} & \beta_{2} & \beta_{3} & \ldots & \beta_{a^{m}-1} \\
\beta_{2} & \alpha_{2} & \beta_{3} & \ldots & \beta_{a^{m}-1} \\
\vdots & & \ddots & & \vdots \\
\beta_{a^{m}-1} & \beta_{a^{m}-1} & \beta_{a^{m}-1} & \ldots & \alpha_{a^{m}-1}
\end{array}\right)
$$

E portanto, como toda matriz de variâncias é definida não negativa, temos que

$$
\begin{aligned}
V\left(X_{t}\right) & =\sum_{i=1}^{a^{m}-1} \alpha_{i} r_{t-i}^{2}+2 \sum_{i=1}^{a^{m}-2} \sum_{j=i+1}^{a^{m}-1} \beta_{j} r_{t-i} r_{t-j}+\sum_{i=0}^{p-1} \gamma_{i} \sigma_{t-i}^{2} \geq \\
& \geq \sum_{i=1}^{a^{m}-1} \alpha_{i} r_{t-i}^{2}+2 \sum_{i=1}^{a^{m}-2} \sum_{j=i+1}^{a^{m}-1} \beta_{j} r_{t-i} r_{t-j} \geq 0,
\end{aligned}
$$


para valores de $\gamma_{i} \geq 0, \forall i=1, \ldots, p$.

Agora vamos demonstrar que existem valores para $\alpha_{j}, \beta_{j}$ e $\gamma_{j}$, tais que $V\left(X_{n}\right)-E\left[V\left(X_{n+1}\right) \mid X_{n}\right] \geq 0, X_{n} \in C^{c}$, onde $C$ é um conjunto compacto.

Primeiro tomemos $\gamma_{0}=1$, então a equação (3.31) fica assim:

$$
\begin{aligned}
V\left(X_{t}\right)-E\left[V\left(X_{t+1}\right) \mid X_{t}\right] & =\sum_{l=0}^{m-1} \sum_{i=a}^{l+1}\left(\alpha_{i}-\alpha_{i+1}-\sum_{j=l+2}^{m+1} C_{j}\right) r_{t-i}^{2}+ \\
& +\sum_{i=1}^{p-1}\left(\gamma_{i}-\gamma_{i+1}-b_{i+1}\right) \sigma_{t-i}^{2}+ \\
& +\left(1-\gamma_{1}-b_{1}-\alpha_{1}-\sum_{i=1}^{m+1} C_{i}\right) \sigma_{t}^{2}-C_{0}
\end{aligned}
$$

Agora consideramos:

$$
\sum_{i=1}^{m+1} a^{i-1} C_{i}+\sum_{k=1}^{p} b_{k}=\sum_{l=0}^{m-1} \sum_{i=a^{l}}^{a^{l+1}-1} \sum_{j=l+2}^{m+1} C_{j}+\sum_{i=1}^{m+1} C_{i}+\sum_{k=1}^{p} b_{k}<1 .
$$

Note-se que temos que:

$$
\alpha_{j}>\sum_{k=j}^{a^{l+1}-1} \sum_{i=l+2}^{m+1} C_{i}+\sum_{s=l+1}^{m-1} \sum_{k=a^{s}}^{a^{s+1}-1} \sum_{i=s+2}^{m+1} C_{i}, \text { para } j=2, \ldots, a^{m}-1
$$

com $l$, tal que $j \in\left\{a^{l}, a^{l}+1, \ldots, a^{l+1}-1\right\}$.

Portanto as seguintes desigualdades são validas:

$$
\begin{gathered}
\alpha_{j}>\sum_{k=j+1}^{a^{l+1}-1} \sum_{i=l+2}^{m+1} C_{i}+\sum_{s=l+1}^{m-1} \sum_{k=a^{s}}^{a^{s+1}-1} \sum_{i=s+2}^{m+1} C_{i}+\sum_{i=l+2}^{m+1} C_{i}, \\
\alpha_{j}-\sum_{i=l+2}^{m+1} C_{i}>\sum_{k=j+1}^{a^{l+1}-1} \sum_{i=l+2}^{m+1} C_{i}+\sum_{s=l+1}^{m-1} \sum_{k=a^{s}}^{a^{s+1}} \sum_{i=s+2}^{m+1} C_{i} .
\end{gathered}
$$

Como a desigualdade (3.35) é estrita, então podemos escolher $\alpha_{j+1}$ satisfazendo (3.34) tal que: 


$$
\alpha_{j}-\sum_{i=l+2}^{m+1} C_{i}>\alpha_{j+1}>\sum_{k=j+1}^{a^{l+1}-1} \sum_{i=l+2}^{m+1} C_{i}+\sum_{s=l+1}^{m-1} \sum_{k=a^{s}}^{a^{s+1}-1} \sum_{i=s+2}^{m+1} C_{i} .
$$

De modo similar para $\gamma_{i}$ temos que:

$$
\gamma_{i}>\sum_{j=i+1}^{p} b_{j}, \text { para } i=2, \ldots, p-1
$$

Portanto as seguintes desigualdades são validas:

$$
\begin{gathered}
\gamma_{i}>\sum_{j=i+2}^{p} b_{j}+b_{i+1}, \\
\gamma_{i}-b_{i+1}>\sum_{j=i+2}^{p} b_{j} .
\end{gathered}
$$

Como a desigualdade (3.38) é estrita, então podemos escolher $\gamma_{i+1}$ satisfazendo (3.37) tal que:

$$
\gamma_{i}-b_{i+1}>\gamma_{i+1}>\sum_{j=i+2}^{n} b_{j}
$$

Agora faltam escolher os valores de $\alpha_{1}$ e $\gamma_{1}$, para isso usamos (3.33)

$$
\begin{aligned}
& \sum_{l=0}^{m-1} \sum_{i=a^{l}}^{a^{l+1}} \sum_{j=l+2}^{m+1} C_{j}+\sum_{i=1}^{m+1} C_{i}+\sum_{k=1}^{p} b_{k}<1 \Rightarrow 1-\sum_{l=0}^{m-1} \sum_{i=a}^{l+1} \sum_{j=l+2}^{m+1} C_{j}-\sum_{i=1}^{m+1} C_{i}-\sum_{k=1}^{p} b_{k}>0 \\
& \Rightarrow 1-\sum_{i=1}^{a-1} \sum_{j=2}^{m+1} C_{j}-\sum_{l=1}^{m-1} \sum_{i=a^{l}}^{a^{l+1}-1} \sum_{j=l+2}^{m+1} C_{j}-\sum_{i=1}^{m+1} C_{i}-\sum_{k=1}^{p} b_{k}>0
\end{aligned}
$$

e, portanto,

$$
1-\sum_{i=1}^{m+1} C_{i}-b_{1}>\sum_{i=1}^{a-1} \sum_{j=2}^{m+1} C_{j}+\sum_{l=1}^{m-1} \sum_{i=a^{l}}^{a^{l+1}} \sum_{j=l+2}^{m+1} C_{j}+\sum_{k=2}^{p} b_{k} .
$$


Como a desigualdade (3.40) é estrita, então podemos escolher $\gamma_{1}$ e $\alpha_{1}$ satisfazendo (3.34) e (3.37), tal que:

$$
1-\sum_{i=1}^{m+1} C_{i}-b_{1}>\alpha_{1}+\gamma_{1}>\sum_{i=1}^{a-1} \sum_{j=2}^{m+1} C_{j}+\sum_{l=1}^{m-1} \sum_{i=a}^{a^{l+1}} \sum_{j=l+2}^{m+1} C_{j}+\sum_{k=2}^{p} b_{k}
$$

Segue das relações (3.41), (3.39) e (3.36) que $\left(\alpha_{i}-\alpha_{i+1}-\sum_{j=l+2}^{m+1} C_{j}\right)>0$ para $i=1, \ldots, a^{m}-1, \quad\left(\gamma_{i}-\gamma_{i+1}-b_{i+1}\right)>0 \quad$ para $\quad i=1, \ldots, p-1 \quad$ e $\left(1-\gamma_{1}-b_{1}-\alpha_{1}-\sum_{i=1}^{m+1} C_{i}\right)>0$. Então $V\left(X_{t}\right)-E\left[V\left(X_{t+1}\right) \mid X_{t}\right]$ pode ser tão grande quanto desejemos se $X_{t}$ está fora de um conjunto compacto.

Portanto, a cadeia

$$
X_{t}=\left(r_{t-1}, \ldots, r_{t-a^{m}+1}, \sigma_{t}, \ldots, \sigma_{t-p+1}\right),
$$

que descreve um processo parcimonioso $\operatorname{GHARCH}(n, p)$, com $n=a^{m}$, é recorrente e tem uma medida invariante.

\section{Proposição 3.4.}

As cadeias de Markov que representam os processos HARCH (2), GHARCH $(2,1)$, GHARCH $(n, p)$ e parcimonioso $\operatorname{GHARCH}(n, p)$, com $n=a^{m}$, são Harris positivas (ergodicidade aperiódica), e cada uma delas possui uma medida de probabilidade invariante (distribuição estacionária), com seu segundo momento finito, para as condições indicadas a seguir.

Prova.

\section{$\underline{\text { HARCH (2) }}$}

A cadeia $X_{t}=\left(r_{t}, r_{t-1}\right)$, onde os valores $r_{t}$ e $r_{t-1}$ são dados pela equação (3.8), descreve um processo HARCH (2).

Sejam $c_{1}$ e $c_{2}$, coeficientes que satisfazem a condição: i) $c_{1}+2 c_{2}<1$, e escolhemos $\alpha$ tal que ii) $\alpha>c_{2}$ e iii) $c_{1}+c_{2}+\alpha<1$. 
Definimos a função $V$ como $V(y, x):=\alpha x^{2}+y^{2}+2 c_{2} x y$.

Temos que

$$
V(y, x)=\alpha x^{2}+y^{2}+2 c_{2} x y \geq c_{2} x^{2}+c_{2} y^{2}+2 c_{2} x y=\left(\sqrt{c_{2}} x+\sqrt{c_{2}} y\right)^{2} \geq 0 .
$$

Então temos que:

$$
V\left(X_{t}\right)-E\left[V\left(X_{t+1}\right) \mid X_{t}\right]=\left(\alpha-c_{2}\right) r_{t-1}^{2}+\left(1-\alpha-c_{1}-c_{2}\right) r_{t}^{2}-c_{0}
$$

Escolhemos $\varpi_{\min }=\min \left(\alpha-c_{2}, 1-\alpha-c_{1}-c_{2}\right)$, e como $\left(\alpha-c_{2}\right)$ e $\left(1-\alpha-c_{1}-c_{2}\right)$ são positivos, temos:

$$
V\left(X_{t}\right)-E\left[V\left(X_{t+1}\right) \mid X_{t}\right] \geq \varpi_{\min } r_{t-1}^{2}+\varpi_{\min } r_{t}^{2}-c_{0} \geq 1-\left(c_{0}+1\right) 1_{B\left(0, \frac{c_{0}+1}{\varpi_{\min }}\right)},
$$

onde $B\left(0, \frac{c_{0}+1}{\sigma_{\min }}\right)$ é a bola com centro zero e raio quadrado $\frac{c_{0}+1}{\bar{\sigma}_{\min }}$.

Portanto, a cadeia $X_{t}=\left(r_{t}, r_{t-1}\right)$ que descreve um processo HARCH (2) é recorrente positiva (ergódica e aperiódica) e tem uma distribuição estacionária.

Agora consideremos $f(x, y)=x^{2}+y^{2}$, então temos,

$$
\begin{aligned}
V\left(X_{t}\right)-E\left[V\left(X_{t+1}\right) \mid X_{t}\right] & \geq \varpi_{\min } r_{t-1}^{2}+\varpi_{\min } r_{t}^{2}-c_{0} \\
& \geq \frac{\varpi_{\min }}{2} r_{t-1}^{2}+\frac{\varpi_{\min }}{2} r_{t}^{2}+\frac{\varpi_{\min }}{2} r_{t-1}^{2}+\frac{\varpi_{\min }}{2} r_{t}^{2}-c_{0} \\
& \geq \frac{\varpi_{\min }}{2} f\left(r_{t-1}, r_{t}\right)-c_{0} 1_{B\left(0, \frac{2 c_{0}}{\varpi_{\min }}\right)}
\end{aligned}
$$

onde $B\left(0, \frac{2 c_{0}}{\sigma_{\min }}\right)$ é a bola com centro zero e raio quadrado $\frac{2 c_{0}}{\bar{\sigma}_{\min }}$.

Portanto, a cadeia $X_{t}=\left(r_{t}, r_{t-1}\right)$ que descreve um processo HARCH (2) tem uma distribuição estacionária com seu segundo momento finito. 


\section{$\underline{\operatorname{GHARCH}(2,1)}$}

A cadeia $X_{t}=\left(\sigma_{t}, r_{t-1}\right)$, onde os valores $r_{t-1}$ e $\sigma_{t}$ são dados pela equação (3.11), descreve um processo GHARCH $(2,1)$.

Sejam $c_{1}, c_{2}$ e $b_{1}$, coeficientes que satisfazem a condição: i) $c_{1}+2 c_{2}+b_{1}<1$, e escolhemos $\alpha$ tal que ii) $\alpha>c_{2}$ e iii) $c_{1}+c_{2}+b_{1}+\alpha<1$.

Definimos a função $V$ como $V(y, x):=\alpha x^{2}+y^{2}$

Temos que, $V(y, x)=\alpha x^{2}+y^{2} \geq 0$.

Então temos que,

$$
V\left(X_{t}\right)-E\left[V\left(X_{t+1}\right) \mid X_{t}\right]=\left(\alpha-c_{2}\right) r_{t-1}^{2}+\left(1-\alpha-c_{1}-c_{2}-b_{1}\right) \sigma_{t}^{2}-c_{0}
$$

Escolhemos $\varpi_{\min }=\min \left(\alpha-c_{2}, 1-\alpha-c_{1}-c_{2}-b_{1}\right)$, e como $\left(1-\alpha-c_{1}-c_{2}-b_{1}\right)$ e $\left(\alpha-c_{2}\right)$ são positivos, temos:

$$
V\left(X_{t}\right)-E\left[V\left(X_{t+1}\right) \mid X_{t}\right] \geq \varpi_{\min } r_{t-1}^{2}+\varpi_{\min } \sigma_{t}^{2}-c_{0} \geq 1-\left(c_{0}+1\right) 1_{B\left(0, \frac{c_{0}+1}{\sigma_{\min }}\right)},
$$

onde $B\left(0, \frac{c_{0}+1}{\sigma_{\min }}\right)$ é a bola com centro zero e raio quadrado $\frac{c_{0}+1}{\bar{\sigma}_{\min }}$.

Portanto, a cadeia $X_{t}=\left(\sigma_{t}, r_{t-1}\right)$ que descreve um processo GHARCH $(2,1)$ é recorrente positiva (ergódica e aperiódica) e tem uma distribuição estacionária. Agora consideremos $f(x, y)=x^{2}+y^{2}$, então temos,

$$
\begin{aligned}
V\left(X_{t}\right)-E\left[V\left(X_{t+1}\right) \mid X_{t}\right] & \geq \varpi_{\min } r_{t-1}^{2}+\varpi_{\min } \sigma_{t}^{2}-c_{0} \\
& \geq \frac{\varpi_{\min }}{2} r_{t-1}^{2}+\frac{\varpi_{\min }}{2} \sigma_{t}^{2}+\frac{\varpi_{\min }}{2} r_{t-1}^{2}+\frac{\varpi_{\min }}{2} \sigma_{t}^{2}-c_{0} \\
& \geq \frac{\varpi_{\min }}{2} f\left(r_{t-1}, \sigma_{t}\right)-c_{0} 1_{B\left(0, \frac{2 c_{0}}{\varpi_{\min }}\right)}
\end{aligned}
$$

onde $B\left(0, \frac{2 c_{0}}{\sigma_{\min }}\right)$ é a bola com centro zero e raio quadrado $\frac{2 c_{0}}{\bar{\sigma}_{\min }}$.

Portanto, a cadeia $X_{t}=\left(\sigma_{t}, r_{t-1}\right)$ que descreve um processo $\operatorname{GHARCH}(2,1)$ tem uma distribuição estacionária com seu segundo momento finito. 


\section{$\underline{\operatorname{GHARCH}(n, p)}$}

A cadeia $X_{t}=\left(r_{t-1}, \ldots, r_{t-n+1}, \sigma_{t}, \ldots, \sigma_{t-p+1}\right)$, onde os valores $r_{t-1}, \ldots, r_{t-n+1}$ e $\sigma_{t}, \ldots, \sigma_{t-p+1}$ são dados pela equação (3.13), descreve um processo $\operatorname{GHARCH}(n, p)$.

Definimos a função $V$, como:

$$
V\left(X_{t}\right)=V\left(r_{t-1}, \ldots, r_{t-n+1}, \sigma_{t}, \ldots, \sigma_{t-p+1}\right):=\sum_{i=1}^{n-1} \alpha_{i} r_{t-i}^{2}+2 \sum_{i=1}^{n-2} \sum_{j=i+1}^{n-1} \beta_{j} r_{t-i} r_{t-j}+\sum_{i=0}^{p-1} \gamma_{i} \sigma_{t-i}^{2}
$$

Então, como tínhamos visto na proposição 3.3, temos que,

$$
\begin{aligned}
V\left(X_{t}\right)-E\left[V\left(X_{t+1}\right) \mid X_{t}\right] & =\sum_{i=1}^{n-2}\left(\alpha_{i}-\alpha_{i+1}-\sum_{j=i+1}^{n} c_{j}\right) r_{t-i}^{2}+\left(\alpha_{n-1}-c_{n}\right) r_{t-n+1}^{2}+ \\
& +\sum_{i=1}^{p-2}\left(\gamma_{i}-\gamma_{i+1}-b_{i+1}\right) \sigma_{t-i}^{2}+\left(\gamma_{p-1}-b_{p}\right) \sigma_{t-p+1}^{2}+ \\
& +\left(1-\gamma_{1}-b_{1}-\alpha_{1}-\sum_{i=1}^{n} c_{i}\right) \sigma_{t}^{2}-c_{0} .
\end{aligned}
$$

Além disso, foi mostrado na proposição 3.3 que,

$$
V\left(X_{t}\right)=\sum_{i=1}^{n-1} \alpha_{i} r_{t-i}^{2}+2 \sum_{i=1}^{n-2} \sum_{j=i+1}^{n-1} \beta_{j} r_{t-i} r_{t-j}+\sum_{i=0}^{p-1} \gamma_{i} \sigma_{t-i}^{2} \geq 0
$$

e que os coeficientes de (3.42) são positivos, isto é,

$$
\begin{aligned}
& \xi_{i}=\left(\alpha_{i}-\alpha_{i+1}-\sum_{j=i+1}^{n} c_{j}\right)>0, i=1, \ldots, n-2, \xi_{n-1}=\left(\alpha_{n-1}-c_{n}\right)>0, \\
& \xi_{n-1+i}=\left(\gamma_{i}-\gamma_{i+1}-b_{i+1}\right)>0, i=1, \ldots, p-2, \xi_{n+p-2}=\left(\gamma_{p-1}-b_{p}\right)>0 \mathrm{e} \\
& \xi_{n+p-1}=\left(1-\gamma_{1}-b_{1}-\alpha_{1}-\sum_{i=1}^{n} c_{i}\right)>0
\end{aligned}
$$

então escolhemos $\varpi_{\min }=\min \left(\xi_{i}\right)$, para $i=1, \ldots, n+p-1$, portanto temos que, 


$$
V\left(X_{t}\right)-E\left[V\left(X_{t+1}\right) \mid X_{t}\right] \geq \varpi_{\min } \sum_{i=1}^{n-1} r_{t-i}^{2}+\varpi_{\min } \sum_{i=0}^{p-1} \sigma_{t-i}^{2}-c_{0} \geq 1-\left(c_{0}+1\right) 1_{B\left(0, \frac{c_{0}+1}{\sigma_{\min }}\right)},
$$

onde $B\left(0, \frac{c_{0}+1}{\sigma_{\min }}\right)$ é a bola com centro zero e raio quadrado $\frac{c_{0}+1}{\sigma_{\min }}$.

Portanto, a cadeia $X_{t}=\left(r_{t-1}, \ldots, r_{t-n+1}, \sigma_{t}, \ldots, \sigma_{t-p+1}\right)$ que descreve um processo $\operatorname{GHARCH}(n, p)$ é recorrente positiva (ergódica e aperiódica) e tem uma distribuição estacionária.

Agora consideremos $f\left(x_{1}, \ldots, x_{n+p-1}\right)=x_{1}^{2}+\ldots+x_{n+p-1}^{2}$, então temos,

$$
\begin{aligned}
V\left(X_{t}\right)-E\left[V\left(X_{t+1}\right) \mid X_{t}\right] & \geq \varpi_{\min } \sum_{i=1}^{n-1} r_{t-i}^{2}+\varpi_{\min } \sum_{i=0}^{p-1} \sigma_{t-i}^{2}-c_{0} \\
& \geq \frac{\varpi_{\min }}{2}\left(\sum_{i=1}^{n-1} r_{t-i}^{2}+\sum_{i=0}^{p-1} \sigma_{t-i}^{2}\right)+\frac{\varpi_{\min }}{2}\left(\sum_{i=1}^{n-1} r_{t-i}^{2}+\sum_{i=0}^{p-1} \sigma_{t-i}^{2}\right)-c_{0} \\
& \geq \frac{\varpi_{\min }}{2} f\left(r_{t-1}, \ldots, r_{t-n+1}, \sigma_{t}, \ldots, \sigma_{t-p+1}\right)-c_{0} 1_{B\left(0, \frac{2 c_{0}}{\varpi_{\min }}\right)}
\end{aligned}
$$

onde $B\left(0, \frac{2 c_{0}}{\sigma_{\min }}\right)$ é a bola com centro zero e raio quadrado $\frac{2 c_{0}}{\bar{\sigma}_{\min }}$.

Portanto, a cadeia $X_{t}=\left(r_{t-1}, \ldots, r_{t-n+1}, \sigma_{t}, \ldots, \sigma_{t-p+1}\right)$ que descreve um processo $\operatorname{GHARCH}(n, p)$ tem uma distribuição estacionária com seu segundo momento finito.

\section{GHARCH $(\boldsymbol{n}, \boldsymbol{p})$ parcimonioso com $n=a^{m}$}

A cadeia $X_{t}=\left(r_{t-1}, \ldots, r_{t-a^{m}+1}, \sigma_{t}, \ldots, \sigma_{t-p+1}\right)$, onde os valores $r_{t-1}, \ldots, r_{t-a^{m}+1} \mathrm{e}$ $\sigma_{t}, \ldots, \sigma_{t-p+1}$ são dados pela equação (3.16), descreve um processo parcimonioso $\operatorname{GHARCH}(n, p) \operatorname{com} n=a^{m}$.

Definimos a função $V$, como:

$$
V\left(X_{t}\right)=V\left(r_{t-1}, \ldots, r_{t-a^{m}+1}, \sigma_{t}, \ldots, \sigma_{t-p+1}\right):=\sum_{i=1}^{a^{m}-1} \alpha_{i} r_{t-i}^{2}+2 \sum_{i=1}^{a^{m}-2} \sum_{j=i+1}^{a^{m}-1} \beta_{j} r_{t-i} r_{t-j}+\sum_{i=0}^{p-1} \gamma_{i} \sigma_{t-i}^{2}
$$

Então, como tínhamos visto na proposição 3.3, temos que, 


$$
\begin{aligned}
V\left(X_{t}\right)-E\left[V\left(X_{t+1}\right) \mid X_{t}\right] & =\sum_{l=0}^{m-1} \sum_{i=a^{l}}^{l+1}-1\left(\alpha_{i}-\alpha_{i+1}-\sum_{j=l+2}^{m+1} C_{j}\right) r_{t-i}^{2}+ \\
& +\sum_{i=1}^{p-1}\left(\gamma_{i}-\gamma_{i+1}-b_{i+1}\right) \sigma_{t-i}^{2}+ \\
& +\left(1-\gamma_{1}-b_{1}-\alpha_{1}-\sum_{i=1}^{m+1} C_{i}\right) \sigma_{t}^{2}-C_{0}
\end{aligned}
$$

Além disso, foi mostrado na proposição 3.3 que,

$$
V\left(X_{t}\right)=\sum_{i=1}^{a^{m}-1} \alpha_{i} r_{t-i}^{2}+2 \sum_{i=1}^{a^{m}-2} \sum_{j=i+1}^{a^{m}-1} \beta_{j} r_{t-i} r_{t-j}+\sum_{i=0}^{p-1} \gamma_{i} \sigma_{t-i}^{2} \geq 0
$$

e que os coeficientes de (3.43) são positivos, isto é,

$$
\begin{aligned}
& \xi_{i}=\left(\alpha_{i}-\alpha_{i+1}-\sum_{j=l+2}^{m+1} C_{j}\right)>0, i=1, \ldots, a^{m}-1, \\
& \xi_{a^{m}-1+i}=\left(\gamma_{i}-\gamma_{i+1}-b_{i+1}\right)>0, i=1, \ldots, p-1 \mathrm{e} \\
& \xi_{a^{m}+p-1}\left(1-\gamma_{1}-b_{1}-\alpha_{1}-\sum_{i=1}^{m+1} C_{i}\right)>0,
\end{aligned}
$$

então escolhemos $\varpi_{\min }=\min \left(\xi_{i}\right)$, para $i=1, \ldots, a^{m}+p-1$, portanto temos que,

$$
V\left(X_{t}\right)-E\left[V\left(X_{t+1}\right) \mid X_{t}\right] \geq \varpi_{\min } \sum_{i=1}^{a^{m}-1} r_{t-i}^{2}+\varpi_{\min } \sum_{i=0}^{p-1} \sigma_{t-i}^{2}-c_{0} \geq 1-\left(c_{0}+1\right) 1_{B\left(0, \frac{c_{0}+1}{\varpi_{\min }}\right)},
$$

onde $B\left(0, \frac{c_{0}+1}{\sigma_{\min }}\right)$ é a bola com centro zero e raio quadrado $\frac{c_{0}+1}{\bar{\sigma}_{\min }}$.

Portanto, a cadeia $X_{t}=\left(r_{t-1}, \ldots, r_{t-a^{m}+1}, \sigma_{t}, \ldots, \sigma_{t-p+1}\right)$ que descreve um processo GHARCH $(n, p)$ com $n=a^{m}$, é recorrente positiva (ergódica e aperiódica) e tem uma distribuição estacionária.

Agora consideremos $f\left(x_{1}, \ldots, x_{a^{m}+p-1}\right)=x_{1}^{2}+\ldots+x_{a^{m}+p-1}^{2}$, então temos, 


$$
\begin{aligned}
V\left(X_{t}\right)-E\left[V\left(X_{t+1}\right) \mid X_{t}\right] & \geq \varpi_{\min } \sum_{i=1}^{a^{m}-1} r_{t-i}^{2}+\varpi_{\min } \sum_{i=0}^{p-1} \sigma_{t-i}^{2}-c_{0} \\
& \geq \frac{\varpi_{\min }}{2}\left(\sum_{i=1}^{a^{m}-1} r_{t-i}^{2}+\sum_{i=0}^{p-1} \sigma_{t-i}^{2}\right)+\frac{\varpi_{\min }}{2}\left(\sum_{i=1}^{a^{m}-1} r_{t-i}^{2}+\sum_{i=0}^{p-1} \sigma_{t-i}^{2}\right)-c_{0} \\
& \geq \frac{\varpi_{\min }}{2} f\left(r_{t-1}, \ldots, r_{t-a^{m}+1}, \sigma_{t}, \ldots, \sigma_{t-p+1}\right)-c_{0} 1_{B\left(0, \frac{2 c_{0}}{\varpi_{\min }}\right)}
\end{aligned}
$$

onde $B\left(0, \frac{2 c_{0}}{\sigma_{\min }}\right)$ é a bola com centro zero e raio quadrado $\frac{2 c_{0}}{\bar{\sigma}_{\min }}$.

Portanto, a cadeia $X_{t}=\left(r_{t-1}, \ldots, r_{t-a^{m}+1}, \sigma_{t}, \ldots, \sigma_{t-p+1}\right)$ que descreve um processo GHARCH $(n, p)$ com $n=a^{m}$, tem uma distribuição estacionária com seu segundo momento finito.

Podemos obter as condições para a existência e esperança incondicional constante de momentos mais altos, mas o cálculo fica crescentemente tedioso. A esperança do $2 \mathrm{~m}$ ésimo momento é:

$$
E\left(r_{t}^{2 m}\right)=E\left(\sigma_{t}^{2 m}\right) E\left(\varepsilon_{t}^{2 m}\right)
$$

Alguns produtos dos retornos, elevados a uma potencia, têm esperança diferente de zero, conduzindo a um sistema de equações para estas esperanças e $E\left(r_{t}^{2 m}\right)$. Por exemplo, para um processo $\operatorname{HARCH}(n)$, o sistema de equações tem a dimensão $m$, para $n=2$, e mais alto para valores de $n$ maiores. No caso relativamente simples do quarto momento $(m=2)$ do $\operatorname{HARCH}(2)(n=2)$, a esperança $E\left(r_{t}^{2} r_{t-1}^{2}\right)$ tem que ser calculada e resolvida junto com a equação para $E\left(r_{t}^{4}\right)$. No caso padrão em que $\varepsilon_{t} \sim N(0,1)$, $E\left(\varepsilon_{t}^{4}\right)=3$ e a seguinte condição necessária é obtida para manter o quarto momento finito,

$$
3\left[c_{2}^{2}+\left(c_{1}+c_{2}\right)^{2}\right]+c_{2}\left[1+3\left(c_{1}^{2}+6 c_{1} c_{2}+4 c_{2}^{2}\right)\right]<1
$$

As condições necessárias e suficientes para o quarto, e em geral o $k$-ésimo momento de um processo $\operatorname{HARCH}(n)$ são dadas em Dacorogna et al. (1996). Processos com um momento de segunda ordem finito e um momento de quarta ordem que diverge podem existir.

Também eles mostram que uma condição explícita necessária para a existência do $2 k$ ésimo momento de um processo $\mathrm{HARCH}(n)$ é, 


$$
\sum_{j=1}^{n}\left(\sum_{i=j}^{n} c_{j}\right)^{k}<\frac{1}{E\left(\varepsilon^{2 k}\right)}
$$

Assim como uma condição explícita suficiente para a existência do $2 k$-ésimo momento de um processo $\operatorname{HARCH}(n)$ é,

$$
\left(\sum_{j=1}^{n} j^{2} c_{j}\right)^{k}<\frac{1}{E\left(\varepsilon^{2 k}\right)} .
$$




\section{Capítulo 4}

\section{Aplicações}

\subsection{Introdução.}

Neste capítulo realizamos aplicações para avaliar o desempenho dos modelos HARCH em séries financeiras. Primeiramente, vamos realizar algumas simulações com o objetivo de entender melhor os modelos HARCH.

Para as estimações dos parâmetros das séries simuladas usaremos o método tradicional de máxima verossimilhança e o método Bayesiano via amostrador de Gibbs, para assim, estabelecer comparações antes de realizar as estimações dos parâmetros de séries reais como a taxa de câmbio Euro-Dólar e o índice Ibovespa.

Alguns documentos sobre inferência Bayesiana sobre processos ARCH podem ser encontrados em Geweke (1989), Kleibergen e van Dijk (1993), Geweke (1994) e Bauwens e Lubrano (1998).

O algoritmo do amostrador de Gibbs é simples quando existem expressões analíticas conhecidas das distribuições condicionais a posteriori dos parâmetros. Quando uma destas densidades condicionais não é conhecida o algoritmo não pode ser usado, pois não se consegue amostrar nas distribuições condicionais desconhecidas. Uma tradicional solução nesta situação é usar o método da rejeição para amostrar na distribuição condicional desconhecida tal como, por exemplo, faz o algoritmo de Metropolis-Hastings.

Uma outra alternativa para solucionar o problema de distribuições condicionais a posteriori desconhecidas é usar o algoritmo do amostrador de Griddy-Gibbs (GriddyGibbs sampler) de Ritter e Tanner (1992). 


\subsection{Amostrador Griddy-Gibbs.}

Seja $\Theta=\left\{\theta_{1}, \ldots, \theta_{n}\right\}$ o conjunto de parâmetros a estimar, e sejam as distribuições condicionais a posteriori conhecidas (que possamos gerar números aleatórios delas), e dadas pela expressão, $\varphi\left(\theta_{i} \mid \theta_{1}, \theta_{2}, \ldots, \theta_{i-1}, \theta_{i+1}, \ldots, \theta_{n}, y\right)$, para cada parâmetro $\theta_{i}$, $i=1, \ldots, n$. O algoritmo do amostrador de Gibbs, veja Besag (1974), Geman e Geman (1984) e Gelfand e Smith (1990), gera uma realização de uma cadeia de Markov $\left\{\theta_{i}^{(n)}\right\}_{n=0}^{N}$, para cada parâmetro $\theta_{i}, i=1, \ldots, n$,

$$
\theta_{i}^{(n)} \sim \varphi\left(\theta_{i} \mid \theta_{1}^{(n-1)}, \ldots, \theta_{i-1}^{(n-1)}, \theta_{i+1}^{(n-1)}, \ldots, \theta_{n}^{(n-1)}, y\right)
$$

O procedimento começa com qualquer valor de $\theta_{2}^{(0)}, \ldots, \theta_{n}^{(0)}$ no suporte da distribuição a posteriori. A influência das condições iniciais desaparece após um certo número de iterações. Se o algoritmo converge depois de $s$ iterações, podemos gerar uma amostra da densidade a posteriori de $\Theta$, a qual é, no entanto, não independente devido à estrutura das cadeias de Markov.

Quando a densidade condicional a posteriori de algum dos parâmetros não é nenhuma densidade conhecida, mas temos uma expressão analítica possível de ser avaliada em uma rede de pontos, podemos usar o amostrador de Griddy-Gibbs, pois podemos calcular a correspondente função de distribuição usando algum método de integração numérica, veja, por exemplo, Davis e Rabinowitz (1975), para depois gerar variáveis aleatórias desta função de distribuição.

Uma técnica de redução de variância é incorporar condicionamento no algoritmo, isto é, usar a média condicional $\sum_{n=1}^{N} E\left(\theta_{i} \mid \theta_{1}^{n}, \ldots, \theta_{i-1}^{n}, \theta_{i+1}^{n}, \ldots, \theta_{n}^{n}, y\right) / N$, para estimar $E\left(\theta_{i} \mid \theta_{1}, \theta_{2}, \ldots, \theta_{i-1}, \theta_{i+1}, \ldots, \theta_{n}, y\right)$, no lugar de $\sum_{n=1}^{N} \theta_{i}^{n} / N$

\subsection{Simulação.}

O primeiro passo é realizar a simulação de alguns processos HARCH para entender o comportamento deles. Para isso foi desenvolvido um código em S-Plus para gerar diversos processos HARCH. O código é o mais geral possível para poder gerar qualquer tipo de processo HARCH.

Na Ilustração 15 podemos ver os retornos e as volatilidades simuladas de vários processos HARCH. Foram geradas 10.000 observações de diversos processos HARCH parcimoniosos e com diferentes níveis de agregações. 

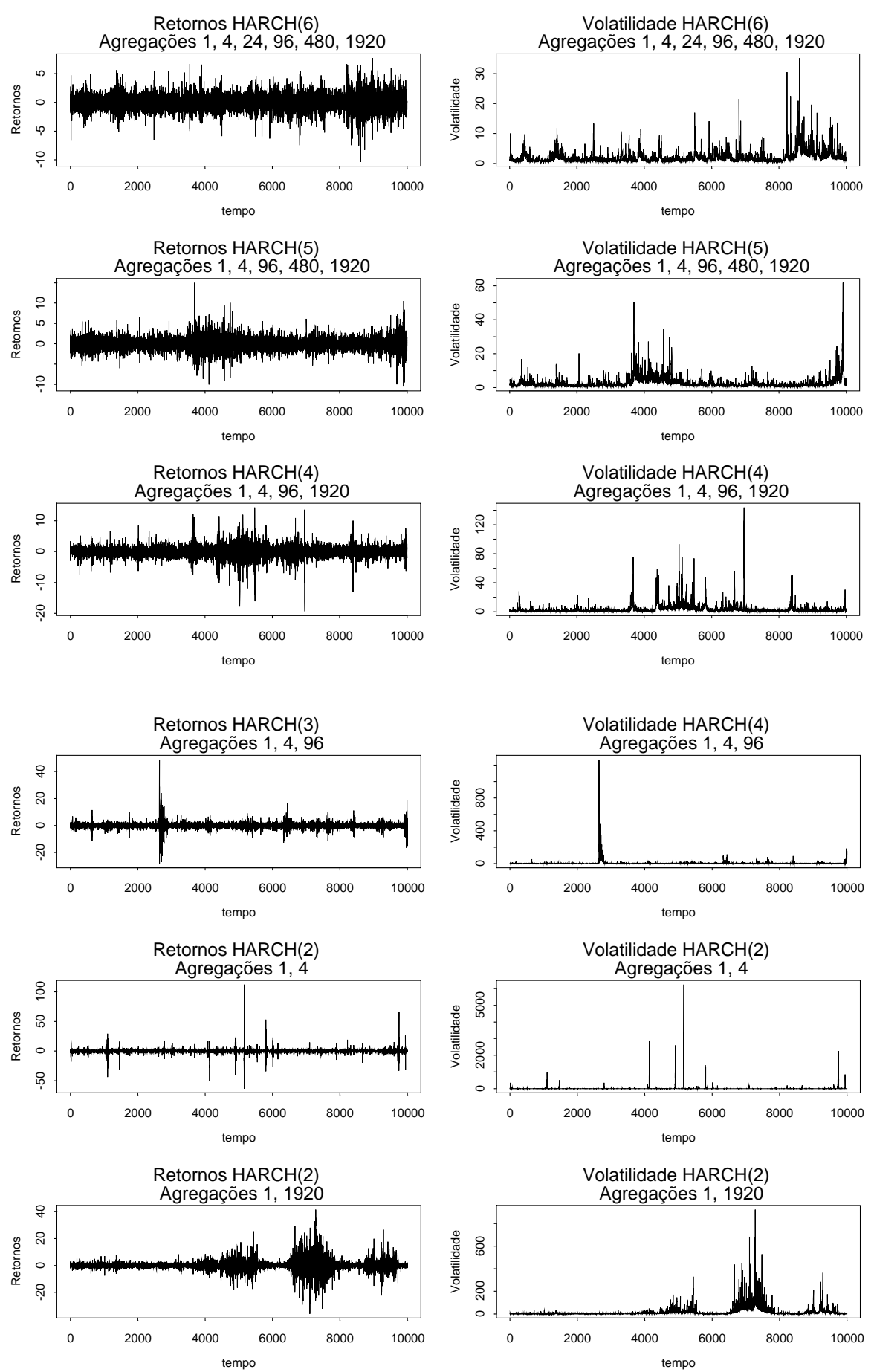

Ilustração 15.- Simulação de processos HARCH parcimoniosos: retornos e volatilidades. 
Os valores dos parâmetros são dados por $C_{0}=0.5$, e $C_{i}=\frac{0.98}{a_{i} n}, i=1, \ldots, n$, onde $n$ é o número de agregações do processo $\operatorname{HARCH}(n)$ e $a_{i}$ é o tamanho de cada agregação. Podem-se ver algumas diferenças importantes entre estes processos como, por exemplo, que cada agregação influencia diretamente nas agrupações das magnitudes dos retornos (clusters de volatilidades), e que quando existem diversos tipos de agrupamentos, cada um perto do outro, como no primeiro gráfico, $\mathrm{HARCH}(6) \mathrm{com}$ agregações 1, 4, 24, 96, 480, 1920, os agrupamentos ficam mais difíceis de serem distinguidos. Esta característica, já tínhamos visto nas séries dos exemplos anteriores e pode ser vista em muitas outras séries financeiras, onde se podem identificar diversos clusters de volatilidades, embora não consigamos identificar nem quantos são nem onde começam ou onde terminam estes clusters.

\subsection{Componentes do Mercado.}

Um dos problemas que aparece, então, é como detectar estes agrupamentos, de que ordem eles são e quantos são. Lamentavelmente não existe nenhuma regra geral para a escolha destes agrupamentos e eles vão depender do comportamento de cada série. Estes agrupamentos são conhecidos como as componentes do mercado, veja Dacorogna et al. (2001).

A escolha do número de componentes e seu tamanho desempenham um papel importante na modelagem, embora seja arbitrária. No entanto, existem algumas regras essenciais que podemos usar, como por exemplo, que o tamanho da agregação seguinte de um processo HARCH $(m)$ parcimonioso tem que ser ao menos o dobro do tamanho da agregação anterior. Para entender melhor, vejamos um exemplo: suponhamos que queremos modelar uma série com um processo HARCH(3) parcimonioso, assim,

$$
\begin{aligned}
r_{t} & =\sigma_{t} \varepsilon_{t} \\
\sigma_{t}^{2} & =C_{0}+C_{1} r_{t-1}^{2}+C_{2}\left(r_{t-1}+\ldots+r_{t-a_{1}}\right)^{2}+C_{3}\left(r_{t-1}+\ldots+r_{t-a_{2}}\right)^{2}
\end{aligned}
$$

e devemos ter que os tamanhos $a_{2} \geq 2 a_{1} \geq 2$. Embora isto não seja obrigatório, ajuda na parcimônia do modelo.

Uma segunda regra é a agregação temporal de processos GARCH vista no capítulo 2. Isto é, fazer varias agregações temporais e olhar quando começam a aparecer desvios significativos entre os parâmetros estimados do processo GARCH e os teóricos da agregação temporal.

Uma outra regra que ajuda a determinar se as componentes escolhidas foram ótimas é o impacto da componente. Define-se o impacto $I_{i}$ da $i$-ésima componente do mercado como 


$$
\begin{aligned}
& I_{1}=C_{1}, \\
& I_{i}=a_{i} C_{i} \quad \forall i>1 .
\end{aligned}
$$

A condição de estacionariedade de um processo $\operatorname{HARCH}(m)$ parcimonioso pode se formulada em termos dos impactos, da seguinte forma:

$$
\sum_{i=1}^{m} I_{i}<1
$$

O número de componentes em uma série financeira pode variar dependendo de como ela é negociada no mercado, assim, séries líquidas podem ter uma estrutura com maior número de componentes que séries não líquidas, pois os agentes do mercado heterogêneo influenciam neste contexto. No nosso caso, as séries do Ibovespa e o Euro poderiam ter diversas componentes que ajudem a modelar o comportamento heterogêneo do mercado de market makers, traders de curto prazo, trades intraday, traders de curto e médio prazo e investidores, por exemplo.

\subsection{Estimações de máxima verossimilhança.}

Quando a função de verossimilhança é complexa ou a quantidade de observações de uma série é muito grande, a estimação de parâmetros por máxima verossimilhança geralmente resulta difícil, é por isso que as implementações de novos algoritmos de otimização ou de métodos Bayesianos para a determinação dos parâmetros são usadas freqüentemente.

Nas secções seguintes vamos a estabelecer comparações entre a estimação de máxima verossimilhança e o amostrador de Griddy-Gibbs. Para isso vamos primeiramente testar a qualidade das estimações de máxima verossimilhança e do simulador de processos HARCH feito no S-Plus.

\begin{tabular}{|l|r|r|}
\hline \multicolumn{3}{|c|}{ HARCH(2) } \\
\hline Parâmetros & \multicolumn{1}{|c|}{ Reais } & Estimados \\
\hline$c_{0}$ & 0.500 & 0.511 \\
& & $(0.07)$ \\
\hline$c_{1}$ & 0.490 & 0.481 \\
& & $(0.10)$ \\
\hline$c_{2}$ & 0.245 & 0.243 \\
& & $(0.05)$ \\
\hline
\end{tabular}

Tabela 5.- Média e desvio padrão das estimações de um processo HARCH(2), com agregações 1 e 2, por máxima verossimilhança, baseadas em 1000 séries simuladas de tamanho 500.

Geramos 1.000 séries simuladas de um processo HARCH(2) com 500 observações cada. O processo simulado foi o seguinte: 


$$
\begin{aligned}
r_{t} & =\sigma_{t} \varepsilon_{t}, \\
\sigma_{t}^{2} & =0.5+0.49 r_{t-1}^{2}+0.245\left(r_{t-1}+r_{t-2}\right)^{2},
\end{aligned}
$$

onde os $\varepsilon_{t} \sim N(0,1)$ são i.i.d.

As estimações de máxima verossimilhança encontram-se na Tabela 5. O processo HARCH simulado tem duas componentes, a primeira de tamanho 1 e a segunda de tamanho 2. Na terceira coluna da tabela encontram-se as médias e os desvios padrões das estimativas de máxima verossimilhança. Pode-se ver a boa qualidade das estimativas. $\mathrm{O}$ algoritmo foi implementado em S-Plus.

Geramos, também, 1.000 séries simuladas de um processo HARCH(2) parcimonioso com agregações de tamanho 1 e 96, com 1000 observações cada série gerada. O processo simulado foi o seguinte:

$$
\begin{aligned}
r_{t} & =\sigma_{t} \varepsilon_{t}, \\
\sigma_{t}^{2} & =0.5+0.49 r_{t-1}^{2}+0.005104\left(r_{t-1}+\ldots+r_{t-96}\right)^{2},
\end{aligned}
$$

onde os $\varepsilon_{t} \sim N(0,1)$ são i.i.d.

\begin{tabular}{|l|r|r|}
\hline \multicolumn{3}{|c|}{ HARCH(2) } \\
\hline Parâmetros & \multicolumn{1}{|l|}{ Reais } & Estimados \\
\hline $\mathbf{C}_{0}$ & 0.500 & 0.505 \\
& & $(0.06)$ \\
\hline $\mathbf{C}_{1}$ & 0.490 & 0.484 \\
& & $(0.07)$ \\
\hline $\mathbf{C}_{2}$ & $5.104 \mathrm{E}-03$ & $5.108-03$ \\
& & $(8.41 \mathrm{E}-04)$ \\
\hline
\end{tabular}

Tabela 6.- Média e desvio padrão das estimações de um processo HARCH(2), com agregações 1 e 96, por máxima verossimilhança, baseadas em 1000 séries simuladas de tamanho 1000.

As estimações de máxima verossimilhança encontram-se na Tabela 6. O processo HARCH simulado tem duas componentes, a primeira de tamanho 1 e a segunda de tamanho 96. Na terceira coluna da tabela encontram-se as médias e os desvios padrões das estimativas de máxima verossimilhança. Pode-se ver a boa qualidade das estimativas. $\mathrm{O}$ algoritmo foi implementado em S-Plus e pode realizar simulações e estimativas de máxima verossimilhança de praticamente qualquer processo HARCH com diversas funções de distribuição das inovações.

Fizemos varias outras simulações e uma observação importante é que quanto maior é o número de agregações e maior é o número de componentes em um processo HARCH parcimonioso, a estimação por máxima verossimilhança fica mais difícil e demorada. 
Nas simulações feitas consideramos coeficientes tais que os impactos de cada componente sejam iguais. Note-se que quanto maior seja o número de agregações de alguma componente, menor será o coeficiente desta componente. Por esse motivo quando queremos ver a significância de alguma componente no modelo é melhor estudar os impactos e não os coeficientes. Isto basicamente deve-se a que os retornos agregados têm geralmente uma magnitude maior que os retornos que não são agregados.

Alem disso, os coeficientes foram escolhidos de tal modo que se cumpram as condições de estacionariedade vistas no capítulo anterior.

Além disso, como já tínhamos mencionado no início desta secção a otimização fica muito difícil quando a função de verossimilhança é complexa.

O algoritmo BHHH de Berndt et al. (1974) ajuda na otimização da função de verossimilhança, pois ele encontra o ótimo global fugindo de cair em ótimos locais. No entanto, quando a função de verossimilhança é praticamente constante em um grande intervalo ele não consegue achar o ótimo global e pode parar em algum valor distante. Os algoritmos genéticos (veja Goldberg, 1989 e Pictet et al., 1995) ou qualquer outro método sofisticado de otimização poderiam ser uma solução para este tipo de problemas. Dacorogna et al. (2001) propõem algoritmo iterativo como uma mistura de algoritmo genético, com crossover e mutação, com o algoritmo BHHH em cada iteração para uma melhor otimização. No entanto, nós tentaremos a utilização de estimação Bayesiana e assim poder compará-la com as estimativas que encontremos por máxima verossimilhança usando métodos tradicionais de otimização.

\subsection{Estimações usando o amostrador Griddy-Gibbs.}

Uma vez comprovada a qualidade do otimizador para o cálculo dos parâmetros de máxima verossimilhança nas simulações anteriores, vamos estudar a qualidade do algoritmo do amostrador Griddy-Gibbs nestas simulações.

$\mathrm{Na}$ verdade, os métodos Bayesianos são menos robustos, no entanto, no método de máxima verossimilhança, quando a função de verossimilhança é muito complexa o otimizador poderia não convergir ao máximo e o algoritmo colapsar ou cair em um looping. Isto pode acontecer ou porque a função de verossimilhança é quase constante em um intervalo grande ou porque os valores dos retornos, no modelo, levam a valores de matrizes "numericamente singulares" dentro do algoritmo de otimização, o que termina causando um colapso no algoritmo e, portanto, o máximo não é encontrado.

Então a proposição de novos algoritmos de otimização, mais sofisticados, ou de novas estratégias do cálculo dos parâmetros do modelo, tal como os métodos Bayesianos, podem ser de muita utilidade e resolver este problema. Vamos estudar as duas propostas para que de agora em diante possa ser utilizada qualquer delas dependendo da complexidade do modelo proposto.

Vamos então simular uma única série, com 2000 observações, de um processo HARCH(2) parcimonioso com agregações de tamanho 1 e 96 . O processo simulado foi o seguinte: 


$$
\begin{aligned}
r_{t} & =\sigma_{t} \varepsilon_{t}, \\
\sigma_{t}^{2} & =0.5+0.49 r_{t-1}^{2}+0.005104\left(r_{t-1}+\ldots+r_{t-96}\right)^{2},
\end{aligned}
$$

onde os $\varepsilon_{t} \sim N(0,1)$ são i.i.d.

No algoritmo de Griddy-Gibbs incorporamos condicionamento para o cálculo dos valores dos parâmetros, isto é, em cada iteração calculamos a esperança condicional do parâmetro. Assim por exemplo para estimar $E\left[C_{0} \mid r_{t}\right]$ usaremos $\sum_{i=1}^{N} E\left[C_{0} \mid C_{1}^{i}, C_{2}^{i}, r_{t}\right] / N$ em lugar de $\sum_{i=1}^{N} C_{0}^{i} / N$, onde $N$ é o total de iterações. Embora ambas as formas de calcular os parâmetros sejam consistentes, o cálculo com condicionamento apresenta menor variância.

Usamos 10000 iterações do algoritmo de Griddy-Gibbs e descartamos as 1000 primeiras.

\begin{tabular}{|l|r|r|r|}
\hline Parâmetro & Real & MV & Griddy-Gibbs \\
\hline \hline $\boldsymbol{C}_{\boldsymbol{0}}$ & 0,50 & 0.52059 & 0.52294 \\
& & & $(0.0322)$ \\
$\boldsymbol{C}_{\boldsymbol{1}}$ & 0,49 & 0,46338 & 0.46629 \\
& & & $(0.0381)$ \\
$\boldsymbol{C}_{\boldsymbol{2}}$ & $5,10417 \mathrm{E}-03$ & $5,35191 \mathrm{E}-03$ & $5.40148 \mathrm{E}-03$ \\
& & & $(4.4903 \mathrm{E}-04)$ \\
\hline $\boldsymbol{N}$ & & & 10000 \\
\hline
\end{tabular}

Tabela 7.- Estimações de um processo $\mathrm{HARCH}(2)$, com agregações 1 e 96, usando o amostrador de Griddy-Gibbs.

Na Tabela 7 podemos ver as estimativas de máxima verossimilhança e usando o amostrador de Griddy-Gibbs da série, com 2000 observações, simulada no S-Plus. A terceira coluna mostra as estimativas por Griddy-Gibbs e seu desvio padrão.

A distribuição dos resíduos considerada foi normal, mas o algoritmo ficou preparado para também rodar com outra distribuição, como por exemplo, $t$. As distribuições a priori, consideradas para os parâmetros foi uniforme. No caso de considerar a distribuição $t(v)$ dos retornos, podemos considerar a distribuição a priori para os graus de liberdade $v$ como uma Cauchy do lado direito centrada em zero, ou uma uniforme ou então escolher subjetivamente o valor dos graus de liberdade. Valores e 5 ou 6 para os graus de liberdade são comumente usados.

Uma das grandes dificuldades no amostrador de Griddy-Gibbs é que se deve estabelecer uma malha de pontos num intervalo para fazer a integração numérica. A determinação errada deste intervalo leva a erros nas estimativas, de igual modo um número pequeno de pontos na malha poderia levar a resultados não satisfatórios. Por outro lado, um número muito grande de pontos na malha pode tornar o algoritmo lento, 
sem necessidade. Um número de 50 pontos na malha, para a estimação e avaliação da distribuição condicional, resultou ser adequado para estimar nosso modelo.

Pelas condições de estacionariedade sabemos que $C_{0}, C_{1}, C_{2} \geq 0$ e $C_{1}+96 C_{2} \leq 1$, então vamos escolher como pontos iniciais de partida do algoritmo $C_{1}=0.5$ e $C_{2}=0.5 / 96$.
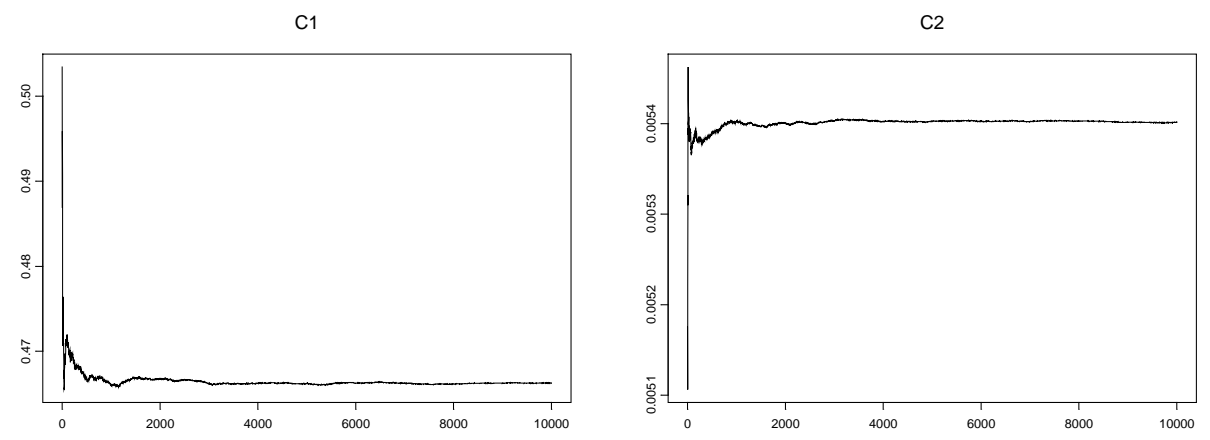

Ilustração 16.- Convergência dos parâmetros $C_{l}$ e $C_{2}$ da simulação do modelo HARCH(2) parcimonioso, com agregações 1 e 96, usando o amostrador Gridd-Gibbs.

Na Ilustração 16 podemos ver a convergência dos parâmetros $C_{1}$ e $C_{2}$ para as 10000 iterações realizadas com o amostrador de Gridd-Gibbs. Podemos notar a proximidade das estimações por Griddy-Gibbs e máxima verossimilhança o que valida o algoritmo de Griddy-Gibbs, embora este último seja muito mais demorado e custoso computacionalmente, mas a principal vantagem é que ele pode substituir máxima verossimilhança quando a função de otimização não converge ao máximo por complexidade da função de verossimilhança.

\section{7. $\quad$ Estimações de máxima verossimilhança para o índice Ibovespa e a taxa de câmbio Euro-Dólar.}

Uma vez testada a qualidade do algoritmo vamos realizar as estimações de máxima verossimilhança para as séries do índice Ibovespa e da taxa de câmbio Euro-Dólar.

Tanto para o índice Ibovespa, quanto para a taxa de câmbio Euro-Dólar dispomos de uma série de dados já tratada, filtrada e agrupada, como visto no capítulo 2.

A série do índice Ibovespa considerada vai desde 2 de janeiro de 1998 ás 11 h00 até 13 de agosto do 2003 ás $17 \mathrm{~h} 00$ em intervalos de 15 minutos, durante dias e horários laborais. Dispõe-se então de 34.449 observações (retornos).

Para a taxa de câmbio Euro-Dólar dispomos de uma série de dados que vai desde 4 de janeiro de 1999 das 00 h00 até 31 de dezembro do 2002 ás 23h30, 24 horas por dia, 
unicamente dos dias úteis, em intervalos de 15 minutos. Dispõe-se então de 96.286 observações (retornos).

O primeiro passo é estabelecer o número de componentes e o número de retornos agregados em cada uma delas. Para ambas séries consideraremos 5 componentes correspondentes a informação de 15 minutos, horária, diária, semanal e mensal.

No caso do Euro-Dólar, então, temos a estimar os parâmetros de um processo HARCH(5) parcimonioso com agregações 1, 4, 96, 480 e 1920, ou seja o modelo ficaria assim:

$$
\begin{aligned}
r_{t}= & \sigma_{t} \varepsilon_{t}, \\
\sigma_{t}^{2}= & C_{0}+C_{1} r_{t-1}^{2}+C_{2}\left(r_{t-1}+\ldots+r_{t-4}\right)^{2}+C_{3}\left(r_{t-1}+\ldots+r_{t-96}\right)^{2}+ \\
& +C_{4}\left(r_{t-1}+\ldots+r_{t-480}\right)^{2}+C_{5}\left(r_{t-1}+\ldots+r_{t-1920}\right)^{2}
\end{aligned}
$$

onde $C_{j} \geq 0, \quad j=1, \ldots, 5, \quad C_{0}>0$, e $\varepsilon_{t} \sim$ i.i.d. $(0,1)$.

No caso do Ibovespa, somente mudam as agregações devido às características da série, então, temos a estimar os parâmetros de um processo HARCH(5) parcimonioso com agregações 1, 4, 24, 120 e 480, ou seja o modelo ficaria assim:

$$
\begin{aligned}
r_{t}= & \sigma_{t} \varepsilon_{t}, \\
\sigma_{t}^{2}= & C_{0}+C_{1} r_{t-1}^{2}+C_{2}\left(r_{t-1}+\ldots+r_{t-4}\right)^{2}+C_{3}\left(r_{t-1}+\ldots+r_{t-24}\right)^{2}+ \\
& +C_{4}\left(r_{t-1}+\ldots+r_{t-120}\right)^{2}+C_{5}\left(r_{t-1}+\ldots+r_{t-480}\right)^{2}
\end{aligned}
$$

\begin{tabular}{|c|c|c|c|c|}
\hline Componente & $\begin{array}{l}\text { Agregações. } \\
\text { Euro-Dólar }\end{array}$ & $\begin{array}{c}\text { Agregações. } \\
\text { lbovespa }\end{array}$ & $\begin{array}{c}\text { Amplitude dos } \\
\text { intervalos }\end{array}$ & $\begin{array}{l}\text { Descrição da } \\
\text { componente }\end{array}$ \\
\hline 1 & 1 & 1 & 15 minutos & $\begin{array}{c}\text { Curto Prazo, negociantes } \\
\text { intradia, oportunidades } \\
\text { de arbitragem, market } \\
\text { makers. }\end{array}$ \\
\hline 2 & 4 & 4 & 1 hora & Negociantes intradia. \\
\hline 3 & 96 & 24 & $1 \mathrm{dia}$ & $\begin{array}{l}\text { Negociantes com } \\
\text { posições diárias. }\end{array}$ \\
\hline 4 & 480 & 120 & 1 semana & $\begin{array}{l}\text { Comerciantes de médio } \\
\text { prazo. } \\
\text { Comerciantes de longo }\end{array}$ \\
\hline 5 & 1920 & 480 & 1 mês & $\begin{array}{l}\text { prazo, negociantes de } \\
\text { derivativos e } \\
\text { investidores. }\end{array}$ \\
\hline
\end{tabular}

onde $C_{j} \geq 0, \quad j=1, \ldots, 5, \quad C_{0}>0$, e $\varepsilon_{t} \sim$ i.i.d. $(0,1)$.

Tabela 8.- Componentes HARCH para o índice Ibovespa e a taxa de câmbio Euro-Dólar. 
O número de parâmetros a estimar, tanto para a série do Euro-Dólar quanto para a série do Ibovespa, é 6, quando $\varepsilon_{t} \sim N(0,1)$ e 7 quando $\varepsilon_{t} \sim t(0,1, v)$, devido à estimação dos $v$ graus de liberdade.

A Tabela 8 mostra as componentes consideradas no modelo HARCH para as séries Euro-Dólar e Ibovespa.

Para a estimação de máxima verossimilhança testamos diversos métodos de otimização no S-Plus ${ }^{13}$, (veja Nash, 1990 e Nocedal e Wright, 1999) tal como o método NelderMead, veja Nelder e Mead (1965), que é um método robusto, trabalha bem com funções não diferenciáveis, mas é um método lento; o método do gradiente conjugado, veja Fletcher e Reeves (1964); o método BFGS (quase Newton, publicado simultaneamente em 1970 por Broyden, Fletcher, Goldfarb e Shanno), veja Dennis et. al. (1981) e Dennis e Mei (1979); o método BFGS com limites superiores e inferiores para os parâmetros, veja Byrd et. al. (1995); o método SANN (simulated annealing), veja Belisle (1992); e o algoritmo Berndt, Hall, Hall e Hausman (BHHH), veja Berndt et al. (1974).

Destes algoritmos os que forneceram resultados mais consistentes foram o L-BFGS-B, SANN e BHHH, que embora sejam demorados não colapsam como acontece com os outros métodos. Estes colapsos geralmente acontecem quando aparecem matrizes numericamente próximas à singularidade.

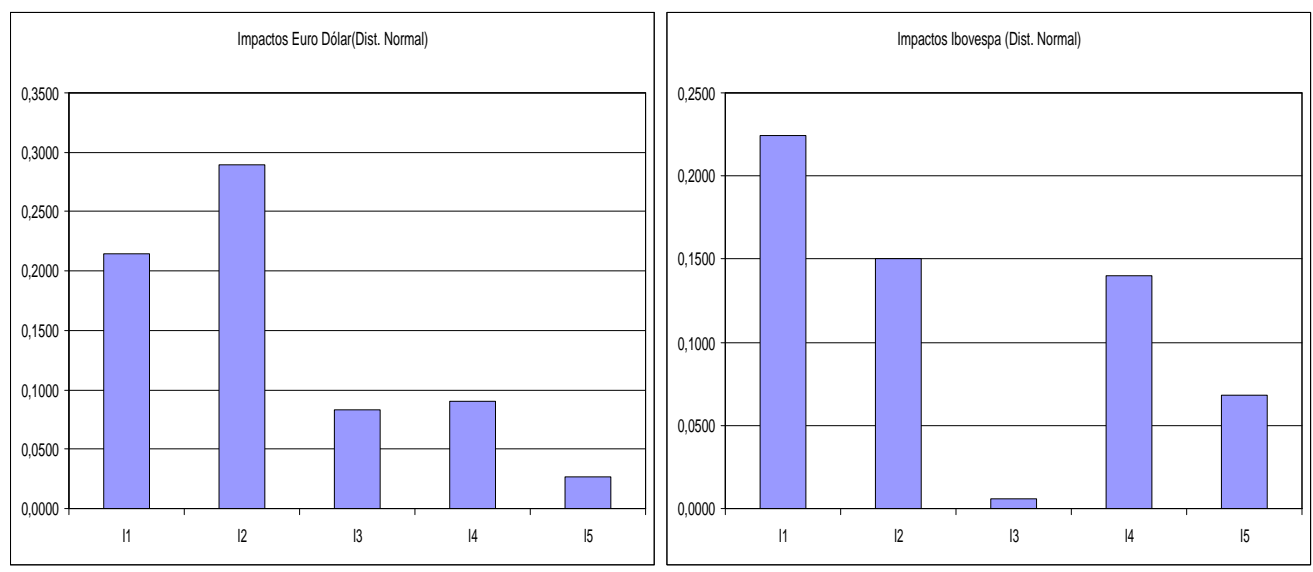

Ilustração 17.- Impactos das componentes, estimados por máxima verossimilhança para a taxa de câmbio Euro-Dólar e o índice Ibovespa.

Os resultados do processo de otimização são um conjunto de coeficientes $C_{j}$ para os quais os impactos são calculados usando a equação (4.1). Como tínhamos mencionado, os coeficientes são difíceis de comparar, pois cada um deles multiplica uma agregação

${ }^{13}$ Usamos a livraria MASS e o modulo finmetrics do S-Plus na elaboração do algoritmo. 
de retornos, e cada agregação destes retornos tem diferente magnitude, assim o que fica mais comparável são os impactos. Em outras palavras poderíamos ter impactos consideráveis ainda quando os coeficientes parecem ser pequenos.

A soma dos impactos deveria ser menor que 1 para ter a estacionariedade dos processos.

$\mathrm{Na}$ Ilustração 17 estão os impactos e coeficientes calculados por máxima verossimilhança para o processo HARCH parcimonioso, para as séries Ibovespa e Euro-Dólar. A distribuição considerada dos resíduos é normal, no entanto, o algoritmo, que foi desenvolvido em S-Plus, é capaz de considerar retornos com distribuição $t$. A propriedade de estacionariedade se cumpre com ambas séries.

Quando a distribuição considerada dos retornos é $t$ tem que se tomar cuidado pois os impactos de um processo HARCH $(m)$ parcimonioso com $m$ agregações de tamanho $a_{1}, \ldots, a_{m}$ mudam, ficando assim:

$$
I_{i}=\frac{v}{v-2} a_{i} C_{i} \quad \forall i \geq 1
$$

Assim a condição de estacionariedade de um processo $\operatorname{HARH}(m)$ parcimonioso fica igual, ou seja,

$$
\sum_{i=1}^{m} I_{i}<1
$$

\begin{tabular}{|c|c|c|c|c|c|}
\hline \multicolumn{6}{|c|}{ Parâmetros lbovespa } \\
\hline $\mathrm{C}_{0}$ & $C_{1}$ & $\mathrm{C}_{2}$ & $\mathrm{C}_{3}$ & $\mathrm{C}_{4}$ & $\mathrm{C}_{5}$ \\
\hline $7,029 \mathrm{E}-06$ & $2,263 E-01$ & $3,964 \mathrm{E}-02$ & $2,328 \mathrm{E}-04$ & $1,162 \mathrm{E}-03$ & 1,432E-04 \\
\hline \multicolumn{5}{|c|}{ Impactos } & \\
\hline$I_{1}$ & $I_{2}$ & $I_{3}$ & $I_{4}$ & $I_{5}$ & Total \\
\hline 0,2263 & 0,1585 & 0,0056 & 0,1395 & 0,0687 & 0,5986 \\
\hline \multicolumn{6}{|c|}{ Parâmetros Euro-Dólar } \\
\hline $\mathrm{C}_{0}$ & $\mathrm{C}_{1}$ & $\mathrm{C}_{2}$ & $\mathrm{C}_{3}$ & $\mathrm{C}_{4}$ & $\mathrm{C}_{5}$ \\
\hline $2,814 \mathrm{E}-07$ & 2,176E-01 & $7,271 \mathrm{E}-02$ & $8,546 \mathrm{E}-04$ & $1,865 \mathrm{E}-04$ & 1,502E-05 \\
\hline \multicolumn{5}{|c|}{ Impactos } & \\
\hline$I_{1}$ & $I_{2}$ & $I_{3}$ & $\mathbf{I}_{4}$ & $\mathbf{I}_{5}$ & Total \\
\hline 0,2176 & 0,2909 & 0,0820 & 0,0895 & 0,0288 & 0,7088 \\
\hline
\end{tabular}

Tabela 9.- Parâmetros e Impactos das componentes, estimados por máxima verossimilhança para a taxa de câmbio Euro-Dólar e o índice Ibovespa. 
Poderíamos, também, calcular a estatística $t$ para determinar quais destes impactos são significativos e, portanto, saber quais são os coeficientes que contribuem para a equação da variância.

Como pode-se apreciar na Tabela 9, os impactos podem ser grandes, embora o tamanho dos parâmetros seja pequeno tal como acontece com a componente semanal do índice Ibovespa.

A importância relativa dos componentes de longo prazo é mais alta para o índice Ibovespa, isto se pode dever a que no índice Ibovespa (bolsa) existe maior número de negociantes de longo prazo que no Euro (moedas), proporcionalmente falando. $\mathrm{Ou}$ seja, a proporção de negociantes intradia no Euro domina o mercado muito mais de que no Ibovespa. Faz muito sentido se considerado o comportamento natural crescente das bolsas.

Em geral os componentes de curto prazo têm maior impacto. Estes componentes de curto prazo modelam essencialmente os negociantes intradia e os market makers, os quais são conhecidos pela sua dominação do mercado.

Os resultados são bastante estáveis para sub amostras de diferentes períodos.

\subsection{Estimações por meio do amostrador Griddy-Gibbs para o índice Ibovespa e a taxa de câmbio Euro-Dólar.}

Como já tínhamos mencionado anteriormente, ter diferentes alternativas de estimação é sempre bom especialmente devido à complexidade da função de verossimilhança, o que pode ocasionar um colapso no momento da estimação. Vamos usar o amostrador de Griddy-Gibbs para a estimação dos parâmetros e assim ver se os resultados teóricos se cumprem na prática. As séries usadas são as mesmas da seção anterior, isto é, as séries do índice Ibovespa e da taxa de câmbio Euro-Dólar, que foram filtradas e agrupadas conforme visto no capítulo 2 .

Para poder realizar uma comparação com o método de máxima verossimilhança, a série do índice Ibovespa considerada vai desde 2 de janeiro de 1998 ás 11 h00 até 13 de agosto do 2003 ás $17 \mathrm{~h} 00$ em intervalos de 15 minutos, durante dias e horários laborais, com 34.449 observações (retornos). Para a taxa de câmbio Euro-Dólar dispomos de uma série de dados que vai desde 4 de janeiro de 1999 das $00 \mathrm{~h} 00$ até 31 de dezembro do 2002 ás 23h30, 24 horas por dia, unicamente dos dias úteis, em intervalos de 15 minutos, com 96.286 observações (retornos).

Os modelos considerados são os mesmos, ou seja, para ambas as séries consideraremos 5 componentes correspondentes a informação de 15 minutos, horária, diária, semanal e mensal.

Os modelos considerados e agregações são os mesmos que no caso da estimação por máxima verossimilhança, ver (4.2) e (4.3).

O número de parâmetros a estimar, tanto para a série do Euro-Dólar quanto para a série do Ibovespa, é 6, quando $\varepsilon_{t} \sim N(0,1)$ e 7 quando $\varepsilon_{t} \sim t(0,1, v)$, devido à estimação dos graus de liberdade. 
A Tabela 8 mostra as componentes consideradas no modelo HARCH para as séries Euro-Dólar e Ibovespa.

\begin{tabular}{|c|c|c|c|c|c|}
\hline \multirow{3}{*}{ Parâmetros } & \multicolumn{4}{|c|}{ Amostrador de Griddy-Gibbs } & \multirow{3}{*}{\begin{tabular}{|l} 
Máxima \\
Verossimilhança \\
Estimação \\
\end{tabular}} \\
\hline & \multicolumn{2}{|c|}{ Normal } & \multicolumn{2}{|c|}{ Condicional } & \\
\hline & Estimação & Desvio Padrão & Estimação & Desvio Padrão & \\
\hline$c_{0}$ & 2.815E-07 & $6.470 \mathrm{E}-09$ & 2.815E-07 & $2.849 \mathrm{E}-09$ & $2.814 \mathrm{E}-07$ \\
\hline$c_{1}$ & 0.21724 & 0.00945 & 0.21768 & 0.00707 & 0.21761 \\
\hline$c_{2}$ & 0.07280 & 0.00242 & 0.07280 & 0.00199 & 0.07271 \\
\hline$c_{3}$ & $8.540 \mathrm{E}-04$ & $5.784 \mathrm{E}-05$ & $8.558 \mathrm{E}-04$ & $4.621 \mathrm{E}-05$ & $8.546 \mathrm{E}-04$ \\
\hline$C_{4}$ & $1.873 \mathrm{E}-04$ & $1.320 \mathrm{E}-05$ & $1.871 \mathrm{E}-04$ & $9.854 \mathrm{E}-06$ & $1.865 \mathrm{E}-04$ \\
\hline$c_{5}$ & 1.507E-05 & $2.994 \mathrm{E}-06$ & $1.509 \mathrm{E}-05$ & $2.120 \mathrm{E}-06$ & $1.502 E-05$ \\
\hline
\end{tabular}

Tabela 10.- Parâmetros estimados do modelo HARCH(5) parcimonioso com agregações 1, 4, 96, 480 e 1920 para a série da taxa de cambio Euro-Dólar, usando o amostrador de Griddy-

Gibbs e máxima verossimilhança.

A estimação usando o amostrador de Griddy-Gibbs é muito demorada e deve ser usada unicamente quando o método de máxima verossimilhança não alcança o ponto de máximo.

Um dos problemas que se tem quando se usa o amostrador de Griddy-Gibbs é a determinação da janela e a quantidade de pontos onde as funções condicionais vão ser avaliadas. Uma escolha errada certamente levara a resultados errados. Para solucionar esse problema pode se estabelecer uma janela observando o intervalo de variação da função de densidade condicional na primeira iteração do amostrador de Griddy-Gibbs e assim estabelecer estes intervalos como a janela de variação para cada parâmetro a estimar.

A Tabela 10 mostra os resultados da estimação do processo HARCH(5) parcimonioso para a taxa de câmbio Euro-Dólar, usando este critério de seleção da janela, para cada parâmetro, onde será avaliada a função de densidade condicional deste parâmetro.

O problema neste caso é se esta janela varia consideravelmente conforme variam os valores dos parâmetros a cada iteração.

Então nós propomos uma modificação no algoritmo de Griddy-Gibbs para solucionar este problema, a saber, durante cada iteração determinamos a janela onde avaliaremos a função de densidade condicional.

Esta janela é determinada pela média e o desvio padrão condicional de cada parâmetro na iteração anterior. Assim a cada iteração nós calculamos e guardamos os resultados da média $M$ e o desvio padrão $S D$ condicionais dos parâmetros a estimar. A janela onde avaliaremos a função de densidade condicional varia a cada iteração e é determinada pelo intervalo desde $M-3 S D$ até $M+3 S D$.

A Tabela 11mostra os resultados da estimação do processo $\mathrm{HARCH}(5)$ parcimonioso para o índice Ibovespa, usando este critério de seleção da janela móvel, para cada parâmetro, onde será avaliada a função de densidade condicional deste parâmetro. 


\begin{tabular}{|c|c|c|c|c|c|}
\hline \multirow{3}{*}{ Parâmetros } & \multicolumn{4}{|c|}{ Amostrador de Griddy-Gibbs } & \multirow{3}{*}{$\begin{array}{l}\text { Máxima } \\
\text { Verossimilhança } \\
\text { Estimação } \\
\end{array}$} \\
\hline & \multicolumn{2}{|c|}{ Normal } & \multicolumn{2}{|c|}{ Condicional } & \\
\hline & Estimação & Desvio Padrão & Estimação & Desvio Padrão & \\
\hline$C_{0}$ & 7.020E-06 & 1.427E-07 & 7.023E-06 & $1.038 \mathrm{E}-07$ & $7.029 \mathrm{E}-06$ \\
\hline$c_{1}$ & 0.22576 & 0.01266 & 0.22651 & 0.01143 & 0.22628 \\
\hline$C_{2}$ & 0.03971 & 0.00241 & 0.03972 & 0.00203 & 0.03964 \\
\hline$c_{3}$ & $2.449 \mathrm{E}-04$ & $1.188 \mathrm{E}-04$ & $2.476 \mathrm{E}-04$ & $1.098 \mathrm{E}-04$ & $2.328 \mathrm{E}-04$ \\
\hline$C_{4}$ & 1.164E-03 & $5.242 \mathrm{E}-05$ & 1.164E-03 & $4.535 \mathrm{E}-05$ & $1.162 \mathrm{E}-03$ \\
\hline$C_{5}$ & $1.444 \mathrm{E}-04$ & 1.025E-05 & 1.439E-04 & $8.290 \mathrm{E}-06$ & $1.432 E-04$ \\
\hline
\end{tabular}

Tabela 11.- Parâmetros estimados do modelo HARCH(5) parcimonioso com agregações 1, 4, 24, 120 e 480 para a série do índice Ibovespa, usando o amostrador de Griddy-Gibbs e máxima verossimilhança.

O número de pontos onde a função de densidade condicional foi avaliada foi 52 . $\mathrm{Na}$ realidade, uma vez determinada a janela de avaliação de maneira adequada, o número de pontos passa a ser pouco importante, isto é, uma malha com 30 ou 50 pontos faz muito pouca diferença no resultado final quando determinada adequadamente a janela onde estes pontos são avaliados.

Os resultados são muito satisfatórios no diagnóstico de convergência, além disso, os parâmetros estimados são praticamente iguais aos obtidos por máxima verossimilhança.

Embora o número de iterações usadas para este cálculo foi somente de 1000 o processo é muito mais demorado que a técnica de máxima verossimilhança.

Como já tínhamos mencionado anteriormente o cálculo usando condicionamento tem menor variância, o que pode ser confirmado tanto na Tabela 10 como na Tabela 11.

\subsection{Previsões para o índice Ibovespa e a taxa de câmbio Euro-Dólar.}

A seguir faremos algumas considerações sobre previsões e validação de modelos. Na realidade, o verdadeiro teste para a veracidade de um modelo de séries temporais é a capacidade de previsão do comportamento futuro da série de interesse. Isto significa que a amostra usada para testar o modelo não será usada na estimação dos parâmetros do modelo. Ou seja, usar duas bases, uma de ajuste, para cálculo das estimativas dos parâmetros do modelo e uma base de validação, onde será avaliado o modelo.

Há uma complexidade adicional no caso de modelos de volatilidade: não se tem uma única definição de volatilidade. Andersen e Bollerslev (1998) mostraram que se estimadores errados da volatilidade são tomados em consideração, então não é possível testar realmente a qualidade da previsão de um modelo. Portanto, é muito importante estabelecer uma estrutura na qual a análise de qualidade da previsão pode ser executada. 
Nesse sentido podemos avaliar a previsão da volatilidade comparando-a com a volatilidade realizada, por exemplo. Ou então poderíamos ver, através de um trading system, como a volatilidade predita ajuda na tomada de decisões. Não entraremos em detalhes sobre os trading systems, mas estes podem ajudar na avaliação das previsões de nossos modelos.

Para poder usar a primeira estratégia de avaliação das previsões, ou seja, compará-las com a volatilidade realizada, precisaríamos realizar a modelagem em uma outra freqüência diferente de 15 minutos, por exemplo, em uma frequiência de uma hora, para assim poder ter retornos para poder calcular a volatilidade realizada a cada hora ${ }^{14}$, ou seja neste caso construiríamos uma série de tempo da volatilidade horária realizada $v_{h}(t)$, a partir de nossa série de tempo dos retornos a cada 15 minutos $^{15}$ (que é nossa menor frequiência para Ibovespa), assim:

$$
v_{h, t}=\sum_{i=1}^{a_{h}} r_{t-i}^{2} .
$$

Aqui, $a_{h}$ é o fator de agregação, neste caso é 4 , correspondente ao número de retornos de 15 minutos que entram no intervalo de 1 hora.

Então, para comparar a volatilidade prevista pelo modelo com a volatilidade realizada, podemos comparar $s_{f}=\tilde{v}_{f, t}-v_{h, t}$, onde $\tilde{v}_{f}$ é a volatilidade predita pelo modelo HARCH, com $s_{r}=v_{h, t+1}-v_{h, t}$. Mais detalhes podem ser encontrados em Taylor e $\mathrm{Xu}$ (1997).

Uma segunda regra de avaliação das previsões poderia ser verificar o seu desempenho através de um trading system, assim, dentre dois modelos, aquele que apresente maior retorno acumulado, num mesmo período de tempo, pode ser considerado como o de maior poder preditivo.

Um trading system pode envolver detalhes como custo por operação, tolerância para a perda (stop loss), retenção de ganhos limites (stop gain) etc.

Analogamente ao que acontece com os modelos GARCH podemos ver como ficam a previsão dos modelos HARCH. Consideremos o processo $\operatorname{GHARCH}(m, p)$ parcimonioso com agregações de tamanhos diferentes $a_{1}, \ldots, a_{m}$, dado por (3.7). A previsão da volatilidade dada pela esperança condicional dada a informação passada fica assim:

$$
\begin{aligned}
& \hat{\sigma}_{t}^{2}(l)=E\left(\sigma_{t+l}^{2} \mid X_{t}\right)=E\left(C_{0}+C_{1}\left(r_{t+l-1}+\ldots+r_{t+l-a_{1}}\right)^{2}+\ldots+\right. \\
& \left.+C_{m}\left(r_{t+l-1}+\ldots+r_{t+l-a_{m}}\right)^{2}+b_{1} \sigma_{t+l-1}^{2}+\ldots+b_{p} \sigma_{t+l-p}^{2} \mid X_{t}\right),
\end{aligned}
$$

\footnotetext{
${ }^{14}$ Isto acontece exclusivamente com a série do índice Ibovespa, pois nossa menor frequiência é 15 minutos.

${ }^{15}$ Poderíamos usar os retornos a cada 5 ou 10 minutos, ao invés de a cada 15 minutos, para o Euro.
} 
onde $X_{t}=\left(r_{t}, \sigma_{t}, r_{t-1}, \sigma_{t-1} \ldots\right)$, para $l=1,2, \ldots$

Como $a_{0}=1<a_{1}<a_{2}<\ldots<a_{m}<\infty$, temos então 3 casos:

- $\quad$ Se $l=1$

$$
\begin{aligned}
\hat{\sigma}_{t}^{2}(l) & =E\left(C_{0}+C_{1}\left(r_{t+l-1}+\ldots+r_{t+l-a_{1}}\right)^{2}+\ldots+C_{s}\left(r_{t+l-1}+\ldots+r_{t+l-a_{s}}\right)^{2}+\ldots+\right. \\
& \left.+C_{m}\left(r_{t+l-1}+\ldots+r_{t+l-a_{m}}\right)^{2}+b_{1} \sigma_{t+l-1}^{2}+\ldots+b_{p} \sigma_{t+l-p}^{2} / X_{t}\right) \\
& =C_{0}+C_{1}\left(r_{t}+\ldots+r_{t+1-a_{1}}\right)^{2}+\ldots+C_{s}\left(r_{t}+\ldots+r_{t+1-a_{s}}\right)^{2}+\ldots+ \\
& +C_{m}\left(r_{t}+\ldots+r_{t+1-a_{m}}\right)^{2}+b_{1} \sigma_{t}^{2}+\ldots+b_{p} \sigma_{t+1-p}^{2}
\end{aligned}
$$

- $\quad$ Se $l$ tal que $a_{s-1}<l<a_{s}, s=1,2, \ldots, m$, então temos,

$$
\begin{aligned}
\hat{\sigma}_{t}^{2}(l)= & E\left(C_{0}+C_{1}\left(r_{t+l-1}+\ldots+r_{t+l-a_{1}}\right)^{2}+\ldots+C_{s}\left(r_{t+l-1}+\ldots+r_{t+l-a_{s}}\right)^{2}+\ldots+\right. \\
& \left.+C_{m}\left(r_{t+l-1}+\ldots+r_{t+l-a_{m}}\right)^{2}+b_{1} \sigma_{t+l-1}^{2}+\ldots+b_{p} \sigma_{t+l-p}^{2} / X_{t}\right) \\
= & E\left(C_{0}+\sum_{i=1}^{s-1} C_{i}\left(\sum_{j=1}^{a_{i}} r_{t+l-j}\right)^{2}+\sum_{i=s}^{m} C_{i}\left(\sum_{j=1}^{l-1} r_{t+l-j}\right)^{2}+\sum_{i=s}^{m} C_{i}\left(\sum_{j=l}^{a_{i}} r_{t+l-j}\right)^{2}+\right. \\
& \left.+\sum_{i=s}^{m} C_{i}\left(\sum_{j=1}^{l-1} r_{t+l-j}\right)\left(\sum_{j=l}^{a_{i}} r_{t+l-j}\right)+b_{1} \sigma_{t+l-1}^{2}+\ldots+b_{p} \sigma_{t+l-p}^{2} / X_{t}\right) \\
= & E\left(C_{0}+\sum_{i=1}^{s-1} C_{i}\left(\sum_{j=1}^{a_{i}} \sigma_{t+l-j} \varepsilon_{t+l-j}\right)^{2}+\sum_{i=s}^{m} C_{i}\left(\sum_{j=1}^{l-1} \sigma_{t+l-j} \varepsilon_{t+l-j}\right)^{2}+\right. \\
& \sum_{i=s}^{m} C_{i}\left(\sum_{j=l}^{a_{i}} r_{t+l-j}\right)^{2}+\sum_{i=s}^{m} C_{i}\left(\sum_{j=1}^{l-1} \sigma_{t+l-j} \varepsilon_{t+l-j}\right)\left(\sum_{j=l}^{a_{i}} r_{t+l-j}\right)+b_{1} \sigma_{t+l-1}^{2}+\ldots+ \\
& \left.+b_{p} \sigma_{t+l-p}^{2} / X_{t}\right),
\end{aligned}
$$

como devido à independência dos $\varepsilon_{t}$ e como $E\left(\varepsilon_{t}\right)=0$, então temos que $E\left(r_{t-i} r_{t-j}\right)=0, \forall i \neq j$, e portanto, 


$$
\begin{aligned}
\hat{\sigma}_{t}^{2}(l)= & E\left(C_{0}+\sum_{i=1}^{s-1} C_{i} \sum_{j=1}^{a_{i}} \sigma_{t+l-j}^{2}+\sum_{i=s}^{m} C_{i} \sum_{j=1}^{l-1} \sigma_{t+l-j}^{2}+\sum_{i=s}^{m} C_{i}\left(\sum_{j=l}^{a_{i}} r_{t+l-j}\right)^{2}+b_{1} \sigma_{t+l-1}^{2}+\ldots+\right. \\
& \left.+b_{p} \sigma_{t+l-p}^{2} / X_{t}\right) \\
& =C_{0}+\sum_{i=1}^{s-1} C_{i} \sum_{j=1}^{a_{i}} \hat{\sigma}_{t+l-j}^{2}+\sum_{i=s}^{m} C_{i} \sum_{j=1}^{l-1} \hat{\sigma}_{t+l-j}^{2}+\sum_{i=s}^{m} C_{i}\left(\sum_{j=l}^{a_{i}} r_{t+l-j}\right)^{2}+b_{1} \tilde{\sigma}_{t+l-1}^{2}+\ldots+ \\
& +b_{p} \tilde{\sigma}_{t+l-p}^{2}
\end{aligned}
$$

onde, para $i=1, \ldots, p$, temos que $\tilde{\sigma}_{t+l-i}^{2}= \begin{cases}\sigma_{t+l-i}^{2}, & i \geq l \\ \hat{\sigma}_{t+l-i}^{2}, & i<l\end{cases}$

- $\quad$ Se $l$ tal que $l>a_{m}, s=1,2, \ldots, m$, então temos,

$$
\begin{aligned}
\hat{\sigma}_{t}^{2}(l) & =E\left(C_{0}+\sum_{i=1}^{m} C_{i} \sum_{j=1}^{a_{i}} \sigma_{t+l-j}^{2}+b_{1} \sigma_{t+l-1}^{2}+\ldots+b_{p} \sigma_{t+l-p}^{2} / X_{t}\right) \\
& =C_{0}+\sum_{i=1}^{m} C_{i} \sum_{j=1}^{a_{i}} \hat{\sigma}_{t+l-j}^{2}+b_{1} \tilde{\sigma}_{t+l-1}^{2}+\ldots+b_{p} \tilde{\sigma}_{t+l-p}^{2}
\end{aligned}
$$

onde, para $i=1, \ldots, p$, temos que $\tilde{\sigma}_{t+l-i}^{2}= \begin{cases}\sigma_{t+l-i}^{2}, & i \geq l \\ \hat{\sigma}_{t+l-i}^{2}, & i<l\end{cases}$ 


\section{Capítulo 5}

\section{Comentários e conclusões}

Os modelos HARCH são de grande valia para a análise de dados de alta freqüência e isso se deve ao fato que os agentes dos mercados financeiros claramente tem um comportamento muito diferente incorporando informação heterogênea no mercado. No entanto, ainda existem problemas principalmente pela complexidade dos algoritmos de estimação.

Um dos grandes desafios, na análise de dados de alta freqüência, é trabalhar com grandes bancos de dados, e modelos muito complexos tendem a apresentar dificuldades computacionais, mesmo com os grandes avanços tecnológicos recentes.

Em particular, a estrutura e estimação dos parâmetros dos modelos HARCH não é tão simples e o custo computacional é a maior desvantagem destes modelos. Neste sentido, o primeiro desafio é estudar técnicas ou processos que ajudem a lidar com esta complexidade computacional, para assim poder desenvolver algoritmos mais eficientes.

Técnicas de estimação dos parâmetros do modelo como máxima verossimilhança podem colapsar tal como já tínhamos descrito no capítulo 4. Então um estudo de otimizadores que sejam robustos neste sentido poderia sugerir uma área de estudo. Atualmente técnicas como algoritmos genéticos ou redes neurais são propostas e discutidas como alternativas de otimização.

Um segundo caminho a seguir são as técnicas Bayesianas. Assim como foi proposto o algoritmo de Griddy-Gibbs no capítulo 4 desta tese, poderia se estender e aprofundar este estudo a outras técnicas alternativas de estimação Bayesiana, estabelecendo-se comparações e recomendações sobre estas diversas técnicas. 
A grande desvantagem do amostrador de Griddy-Gibbs é o tempo elevado deste algoritmo pelo grande esforço computacional. Melhoras neste sentido também seriam interessantes ou então alternativas equivalentes, Bayesianas ou clássicas.

De outro ponto de vista, poderiam ser desenvolvidos modelos mais sofisticados que levem em conta a importância da chegada da informação nos modelos de volatilidade. Assim por exemplo, poderíamos pensar no desenvolvimento de algum tipo de modelo de Volatilidade Estocástica que leve em consideração a heterogeneidade na chegada da informação por parte dos agentes do mercado, a viabilidade destes modelos e estudos teóricos de ergodicidade, estacionariedade etc.

Outra possível alternativa poderia ser considerar modelos não paramétricos, por exemplo, adaptar os modelos CHARN à característica de heterogeneidade da informação.

Modelos semi-paramétricos podem ajudar não somente como um campo novo de estudo de modelos com incorporação de informação heterogênea, mas também podem ajudar a entender melhor a microestrutura do mercado, a maneira como a informação heterogênea pode ser incluída na modelagem etc.

Por último, assim como foram propostas diferentes metodologias e extensões para os processos GARCH, como por exemplo GARCH IN MEAN (Bollerslev, 1986), LGARCH (Taylor, 1986), MGARCH (Geweke e Pantula, 1986), IGARCH (Engle e Bollerslev, 1986), EGARCH (Nelson, 1991), TS-GARCH (Schwert, 1989), T-GARCH (Gourieroux, 1992 e Zakonian, 1994), A-PARCH (Ding et. al., 1993), Q-GARCH (Sentana, 1991), HARCH (Müller et. al., 1997), etc., poderiam ser estudadas estas mesmas metodologias ou adaptá-las para estender os processos HARCH.

Uma das características pouco atrativas dos modelos HARCH estudados é que as componentes do mercado são tomadas de maneira totalmente subjetiva, isto é, a quantidade de agentes que estão no mercado financeiro e trazem informação heterogênea para a formação dos processos de volatilidade, e que é modelada nos processos HARCH. Assim, por exemplo, para as séries do Ibovespa e do Euro-Dólar, consideramos 5 componentes com diferentes agregações cada, tal como citamos no capítulo 4. A escolha destas componente foi arbitrária e logicamente poderia ter sido escolhido um número diferente de componentes e agregações de cada componente.

Algumas propostas para a escolha destes componentes foram dadas no capítulo 4 desta tese, mas um maior aprofundamento e novas propostas poderiam ser um tema de estudo interessante.

Por último, de nada servem diferentes estratégias de estimação da volatilidade se o desempenho e o ganho que estas trazem não é avaliado, e este é um ponto a criticar na grande maioria de trabalhos da área estatística. Uma proposta de uma nova técnica tem maior relevância quando é comparada com outras e são estudadas as vantagens e desvantagens que cada uma delas oferece, como no nosso caso mostramos as comparações entre os modelos GARCH e HARCH.

Desde um ponto de vista menos teórico, esta comparação poderia ser a avaliação na previsão de cada modelo, embora, isto depende sempre do período de estudo analisado e das séries estudadas. 
Neste sentido os modelos de volatilidade podem ser desenvolvidos para cálculo do VaR ou para estabelecer estratégias de negociação. Uma avaliação do desempenho do modelo pode ser interessante.

Não somente uma avaliação dos resíduos, mas uma avaliação da performance do modelo na previsão, assim como foi proposto no capítulo 4 . Igualmente diferentes técnicas de negociação (trading systems) que usem estas metodologias podem ser de utilidade e a avaliação dos retornos acumulados que cada uma delas oferece seria o aspecto fundamental na comparação das diferentes técnicas. Mais detalhes podem ser encontrados em Acar e Satchell, (2002), Dunis, et. al., (2003), Ghysels e Jasiak, (1994) e Park e Irwin, (2005). 


\section{Referências bibliográficas}

[1]. Acar, E. and Satchell, S. (2002). Advanced Trading Rules, Oxford: ButterworthHeinemann.

[2]. Andersen, T. G., and Bollerslev, T. (1997). Heterogeneous Information Arrivals And Return Volatility Dynamics: Uncovering the Long-Run in High Frequency Returns. Journal of Finance, 52, 975-1005.

[3]. Andersen, T. G., and Bollerslev, T. (1998). Answering The Skeptics: Yes, Standard Volatility Models Do Provide Accurate Forecasts. International Economic Review, 39, 885-905.

[4]. Baillie, R. T., Bollerslev, T., and Mikkelsen, H. O. (1996). Fractionally Integrated Generalized Autoregressive Conditional Heteroskedasticity. Journal of Econometrics, 74, 3-30.

[5]. Bauwens, L. and Lubrano, M. (1998). Bayesian Inference On GARCH Models Using The Gibbs Sampler. Econometrics Journal, 1, 23-46.

[6]. Belisle, C. J. P. (1992). Convergence Theorems For A Class Of Simulated Annealing Algorithms On $\mathrm{R}^{\wedge} \mathrm{d}$. Journal of Applied Probability, 29, 885-895. 
[7]. Berndt, E., Hall, B., Hall, R., and Hausman, J. (1974). Estimation And Inference In Nonlinear Structural Models. Annals of Economic and Social Measurement, 3, 653-665.

[8]. Besag, J. (1974). Spatial Interaction And The Statistical Analysis Of Lattice Systems (With Discussion). Journal of Royal Statistical Society, Series B 36, 192-236.

[9]. Bollerslev, T. (1986). Generalized Autoregressive Conditional Heteroskedasticity. Journal of Econometrics, 31, 307-327.

[10]. Bollerslev, T., and Wooldrigde, J. M. (1992). Quasi-Maximum Likelihood Estimation And Inference In Dynamic Models With Time Varying Covariances. Econometric Reviews, 11, 143-172.

[11]. Byrd, R. H., Lu, P., Nocedal, J. and Zhu, C. (1995). A Limited Memory Algorithm For Bound Constrained Optimization. SIAM J. Scientific Computing, 16, 1190-1208.

[12]. Clark, P. (1973). A Subordinated Stochastic Process Model With Finite Variance For Speculative Price. Econometrica, 41, 135-156.

[13]. Dacorogna, M. M., Müller, U. A., Embrechts, P., and Samorodnitsky, G. (1996). Moment Condition For HARCH(K) Models? A discussion paper by the O\&A Research Group.

[14]. Dacorogna, M. M., Embrechts, P., Müller, U. A., and Samorodnitsky, G. (1998). How Heavy Are The Tails Of A Stationary HARCH(K) Processes? Stochastic Processes and Related Topics Y. Rajput and M. Taqqu, Birkhäuser, Boston.

[15]. Dacorogna, M. M., Gençay, R., Müller, U. A., Olsen, R. V., and Pictet, O. V. (2001). An Introduction To High-Frequency Finance, San Diego: Academic Press.

[16]. Dacorogna, M. M., Müller, U. A., Nagler, R. J., Olsen, R. B., and Pictet, O. V. (1993). A Geographical Model For The Daily And Weekly Seasonal Volatility In The FX Market. European Journal of Finance, 1, 243-253.

[17]. Davis , P. J. and Rabinowitz, P. (1975). Methods Of Numerical Integration. New York: Academic Press.

[18]. Dennis, J. E., Gay, D. M., and Welsch, R. E. (1981). An Adaptive Nonlinear Least-Squares Algorithm. ACM Transactions on Mathematical Software 7, 348383. 
[19]. Dennis, J. E. and Mei, H. H. W. (1979). Two New Unconstrained Optimization Algorithms Which Use Function And Gradient Values. Journal of Optimization Theory and Applications 28, 453-483.

[20]. Diebold, F. X. (1988). Empirical Modeling Of Exchange Rates, New York: Springer Verlag.

[21]. Ding, Z., Granger, C. W. J., and Engle, R. F. (1993). A Long Memory Property Of Stock Market Returns And A New Model. Journal of Empirical Finance, 1, 83-106.

[22]. Drost, F. and Nijman, T. (1992). A Test Of The Validity Of A GARCH Diffusion Limit. Mimeographed, Tilburg University.

[23]. Drost, F. and Nijman, T. (1993). Temporal Aggregation Of GARCH Processes. Econometrica, 61, 909-927.

[24]. Duda, R. O., Hart, P. E., Stork, D. G. (2001). Pattern Classification, New York: John Wiley \& Sons, Inc.

[25]. Dunis, C., Laws, J., Naïm, P. (2003). Applied Quantitative Methods For Trading And Investment, London: The Wiley Finance Series.

[26]. Engle, R. F. (1982). Autoregressive Conditional Heteroskedasticity With Estimates Of The Variance Of U. K. Inflation. Econometrica, 50, 987-1008.

[27]. Engle, R. F., Bollerslev, T. (1986). Modelling The Persistence Of Conditional Variance. Econometric Reviews 5, 1-50, 80-87.

[28]. Fletcher, R. and Reeves, C. M. (1964). Function Minimization By Conjugate Gradients. Computer Journal 7, 148-154.

[29]. Gelfand, A. E. and Smith A. F. M. (1990). Sampling-Based Approaches To Calculating Marginal Densities. Journal of the American Statistical Association, 85, 398-409.

[30]. Geman, S. and Geman, D. (1984). Stochastic Relaxation Gibbs Distribution And The Bayesian Restoration Of Images. IEEE Transactions on Pattern Analysis and Machine Intelligence. 6, 721-741.

[31]. Geweke, J., and Pantula, S. (1986). Modelling The Persistence Of Conditional Variances: A Comment. Econometric Reviews, 5, 57-61, 71-74.

[32]. Geweke, J. (1989). Exact Predictive Densities In Linear Models With ARCH Disturbances. Journal of Econometrics, 40, 63-86. 
[33]. Geweke, J. (1994). Bayesian Comparison Of Econometric Models. Working Paper 532, Research Department, Federal Reserve Bank of Minneapolis.

[34]. Ghysels, E. e J. Jasiak (1994). Stochastic Volatility And Time Deformation; An Apllication To Trading Volume And Leverage Effects. Artigo apresentado na Western Finance Association Meeting, Santa Fé.

[35]. Goldberg, D. E. (1989). Genetic Algorithms In Search, Optimization \& Machine Learning, Massachusetts: Addison-Wesley.

[36]. Gourieroux, C. (1992). Qualitative Threshold ARCH Models. Journal of Econometrics, 52, 159-199.

[37]. Hamilton, J. D. (1994). Time Series Analysis, Princeton University Press, New Jersey: Princeton.

[38]. Hull, J. (1995). Introduction To Futures And Options Markets, $2^{\text {nd }}$ ed., New Jersey: Prentice Hall, Inc.

[39]. Kleibergen, F. and van Dijk, H.K. (1993). Non-Stationarity In GARCH Models: A Bayesian Analysis. Journal of Applied Econometrics, 8 (Supplement), 41-61.

[40]. Mandelbrot, B. e H. Taylor (1967). On The Distribution Of Stock Prices Differences. Operations Reserach, 15, 1057-1062.

[41]. Meyn, S. P. and Tweedie, R. L. (1996). Markov Chains And Stochastic Stability, London: Springer Verlag.

[42]. Müller, U. A., Dacorogna, M. M., Davé, R. D., Olsen, R. B., Pictet, O. V., and von Weizsäcker, J. E. (1997). Volatilities Of Different Time Resolutions Analyzing The Dynamics Of Market Components. Journal of Empirical Finance, 4, 213-289.

[43]. Nash, J. C. (1990). Compact Numerical Methods For Computers. Linear Algebra And Function Minimisation. Bristol: Adam Hilger.

[44]. Nelder, J. A. and Mead, R. (1965). A Simplex Algorithm For Function Minimization. Computer Journal 7, 308-313.

[45]. Nelson, D. B. (1991). Conditional Heterocedasticity In Asset Returns: A New Approach. Econometrica, 59, 347-370.

[46]. Nelson, D. B., and Foster, D. P. (1994). Asymptotic Filtering Theory For Univariate ARCH Models. Econometrica, 62, 1-41. 
[47]. Nocedal, J. and Wright, S. J. (1999). Numerical Optimization. New York: Springer.

[48]. Park, C. H., Irwin, S. H. (2005). The Profitability Of Technical Trading Rules In US Futures Markets: A Data Snooping Free Test. AgMAS Project Research Report.

[49]. Pesaran, M. H., and Timmerman, A. (1992). A Simple Nonparametric Test Of Predictive Performance. Journal of Business and Economic Statistics, 10, 461465.

[50]. Pictet, O. V., Dacorogna, M. M., Chopard, B., Oussaidène, M., Schirru, R., and Tomassini, M. (1995). Using Genetic Algorithms For Robust Optimization In Financial Applications. Neural Network World, 5, 573-587.

[51]. Ritter, C. and Tanner, M. A. (1992). Facilitating The Gibbs Sampler: The Gibbs Stopper And The Griddy-Gibbs Sampler. Journal of the American Statistical Association, 87, 861-868.

[52]. Schwert, G. W. (1989). Why Does Stock Market Volatility Change Over Time? Journal of Finance, 44, 1115-1153.

[53]. Sentana, E. (1991). Quadratic ARCH Models: A Potential Reinterpretation Of ARCH Models. LSE Financial Markets Group Discussion Paper 122.

[54]. Taylor, S. J. (1986). Modelling Financial Time Series, New York: Wiley.

[55]. Taylor, S. J., and Xu, X. (1997). The Incremental Volatility Information In One Million Foreign Exchange Quotations. Journal of Empirical Finance, 4, 317340.

[56]. Theodoridis, S., Koutroumbas, K. (2006). Pattern Recognition, San Diego: Academic Press.

[57]. Tsay, R. S. (2002). Analysis Of Financial Time Series, New York: John Wiley \& Sons, Inc.

[58]. Vidyamurthy, G. (2004). Pairs Trading: Quantitative Methods And Analysis, New Jersey:Wiley Finance.

[59]. Weiss, A. A. (1986). Asymptotic Theory Of ARCH Models: Estimation And Testing. Econometric Theory, 1, 352-362.

[60]. White, H. (1980). A Heteroscedasticity-Consistent Covariance Matrix And A Direct Test For Heteroscedasticity. Econometrica, 48, 421-448. 
[61]. Zakonian, J. M.(1994). Threshold Heteroskedastic Models. Journal of Economic, Dynamics and Control, 18, 931-955.

[62]. Zumbach, G. (2000). The Pitfalls In Fitting GARCH Processes. Advances in Quantitative Asset Management. Amsterdam, C. Dunis, [Ed.]. 
This document was created with Win2PDF available at http://www.daneprairie.com. The unregistered version of Win2PDF is for evaluation or non-commercial use only. 